Portland State University

PDXScholar

$12-16-2021$

\title{
Antipsychotic Medication Administration in Oregon Assisted Living/Residential Care Settings: Analyzing an Action Situation
}

Sarah Dys

Portland State University

Follow this and additional works at: https://pdxscholar.library.pdx.edu/open_access_etds

Part of the Community Health Commons, Gerontology Commons, and the Public Health Commons Let us know how access to this document benefits you.

\section{Recommended Citation}

Dys, Sarah, "Antipsychotic Medication Administration in Oregon Assisted Living/Residential Care Settings: Analyzing an Action Situation" (2021). Dissertations and Theses. Paper 5861.

https://doi.org/10.15760/etd.7732

This Dissertation is brought to you for free and open access. It has been accepted for inclusion in Dissertations and Theses by an authorized administrator of PDXScholar. Please contact us if we can make this document more accessible: pdxscholar@pdx.edu. 
Antipsychotic Medication Administration in Oregon Assisted Living/Residential Care Settings: Analyzing an Action Situation

by

Sarah Dys

A dissertation submitted in partial fulfillment of the requirements for the degree of

\author{
Doctor of Philosophy \\ in \\ Community Health
}
Dissertation Committee:
Paula Carder, Chair
Dawn M. Richardson
Glenise McKenzie
Ozcan Tunalilar

Portland State University

2021 


\begin{abstract}
Antipsychotic medication use (APU) in assisted living and residential care (AL/RC) settings is an under-studied and controversial health policy issue. APU in older adults with dementia is associated with an increased risk of falls, hospitalizations, and early mortality. I operationalize the Institutional Analysis and Development Framework using a situational analysis approach, an extension of grounded theory methods, to explore the APU in Oregon AL/RC settings. Regulatory deficiency citations, Oregon AL/RC population data, and semi-structured interviews suggest that staff role clarity, organizational characteristics, and perceived agency influence decision-making around APU. AL/RC providers and caregivers are forced to simultaneously balance and prioritize regulatory goals, organizational constraints, and complex care provision resulting in a multilayered, complicated, and unique situation. Findings highlight the need for creative policy and population health-minded solutions that reflect the practical reality of providing care in AL/RC settings to promote quality of life for residents.
\end{abstract}




\section{Dedication}

I am deeply honored that I was brought into this world into the loving arms of an intergenerational family. I had the privilege of knowing all four of my grandparents and have relationships with many great-aunts and great-uncles. This dissertation is dedicated to all of the loving, sage older adults in my life. Specifically, to my grandparents Lamia Hajjar (Sitto), Khalil Touma (Jiddo), Theodora Zouvelos Dys, and Frank Dys (Grandpa). They are the "why" behind my pursuit of gerontology. 


\section{Acknowledgements}

In addition to the countless hours of labor poured into this study, this work exists because of the generosity, kindness, and mentorship of so many. To my parents Dr. Rabiah Dys and Michael Dys who unconditionally provide me with love, support, encouragement, resources, and advice. My husband, Dr. Behzad Ahmadi for your partnership and unwavering love and care, for pushing me to finish this degree. To future doctors Anna Steeves-Reece and Lindsey Smith, my "faux-hort"; without your solidarity and support, I would not have made it through this program. I am forever grateful for the investment and caring of my dissertation chair, advisor, mentor, and reason for choosing Oregon Health and Science University-Portland State University's School of Public Health: Dr. Paula Carder and look forward to sustaining our relationship into the future. Thank you to my committee, Dr. Dawn Richardson, Dr. Glenise McKenzie, and Dr. Ozcan Tunalilar, who have generously provided their time and energy to nurture me as a budding scholar. Many thanks to Dr. Keren Brown-Wilson and Dr. Michael DeShane whose leadership in the aging field and support of PSU's Institute on Aging resulted in a fellowship that supported this work. To my colleagues and counterparts at the Institute on Aging, I am forever grateful I had the opportunity to have developed my research skills among such amazing people. Thank you to my external stakeholder committee, who offered their expertise and feedback over the course of this dissertation study. Additionally, Dr. Charlie Waters, a geriatric-trained pharmacist provided significant insights to the design and pursuit of the research question. Thank you all, and the many others who have impacted my life and work. I could fill a book with your names! 
Table of Contents

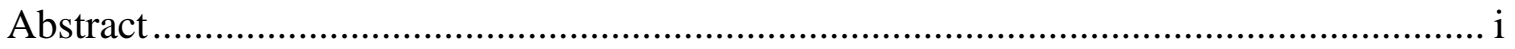

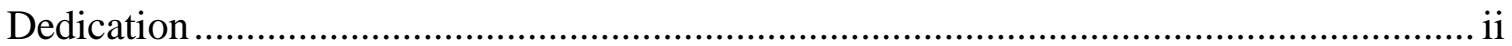

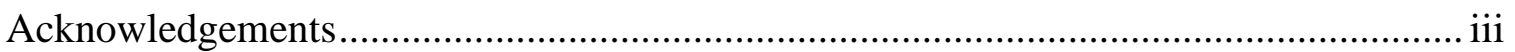

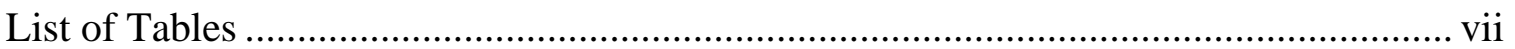

List of Figures .............................................................................................. viii

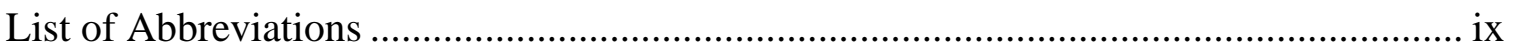

Chapter 1. Introduction and Research Aims ............................................................... 1

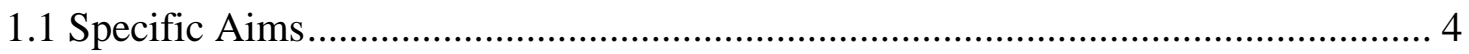

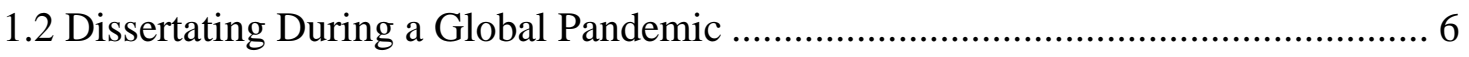

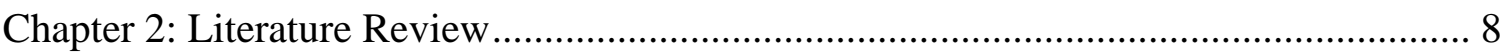

2.1 Assisted Living as a Long-Term Services and Supports Option ........................... 9

2.2 Behavioral Expressions in Older Adults with Dementia or Cognitive Impairment 10

2.3 Responding to Behavioral Expressions Associated with Dementia or Cognitive

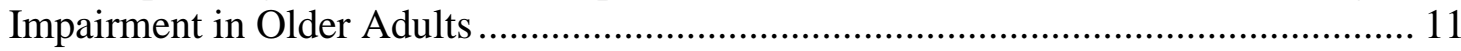

2.4 Antipsychotic Medications Use Disparity in Older Adults with Dementia........... 13

2.5 Assisted Living/Residential Care Context in Oregon ........................................ 14

2.6 Medication Management in AL/RC Settings................................................. 18

2.7 Institutional Analysis and Development (IAD) Framework................................ 19

2.8 Situational Analysis: Examining Social Worlds ............................................... 23

Chapter 3: Paperwork, Paradox, and Pro Re Nata: Psychotropic Medication Deficiencies

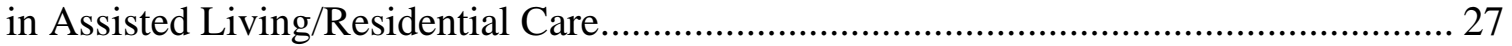

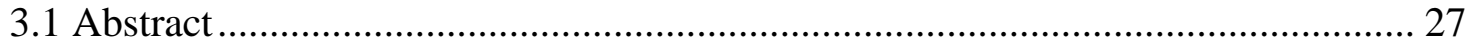

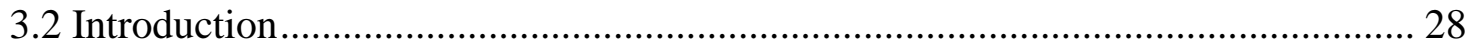

3.2.1 Psychotropic Medication Deficiency Citations: What Can We Learn? .......... 28

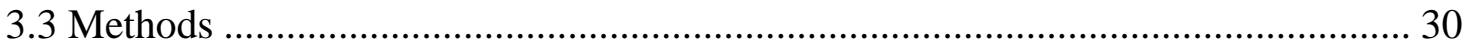

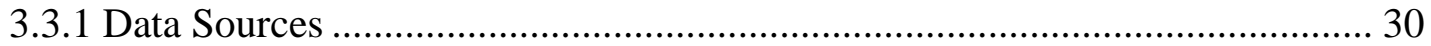

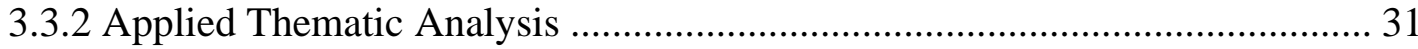

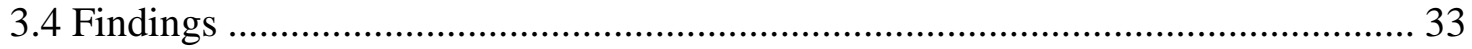

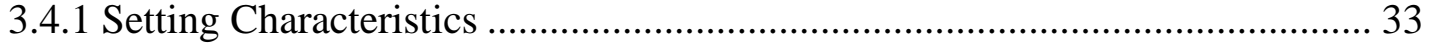

3.4.2 Theme 1: Resident records lacked detailed documentation of administration instructions and attempted nonpharmacologic interventions ............................... 33

3.4.3 Theme 2: Unclear parameters place direct care workers in a role paradox..... 35 
3.4.4 Theme 3: Disconnect regarding when to seek qualified expertise prior to requesting psychotropic medication from residents' primary care providers............ 36

3.4.5 Theme 4: Going through the motions or driving change: Who are plans of correction for? .............................................................................................. 38

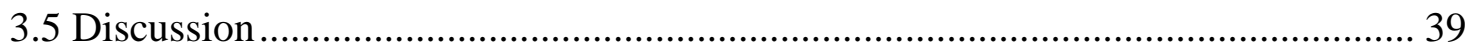

3.5.1 Limitations \& Future Directions .................................................................... 43

3.5.2 Conclusions \& Implications....................................................................... 44

Chapter 4: Antipsychotic Medication Use in Assisted Living/Residential Care: Do Organizational Characteristics Matter?....................................................................... 45

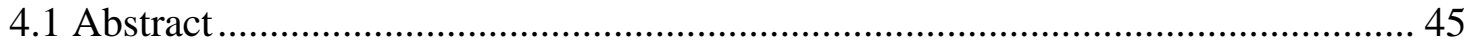

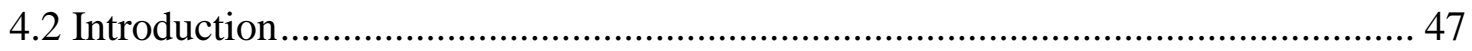

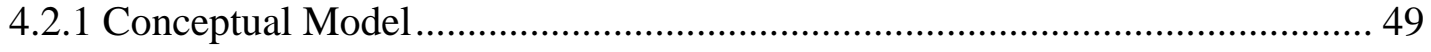

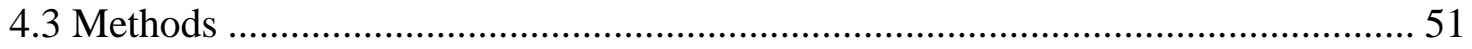

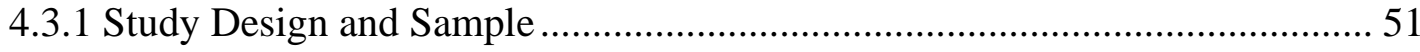

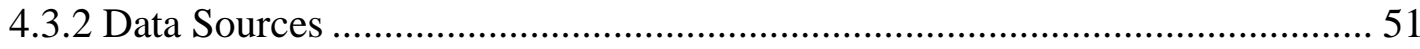

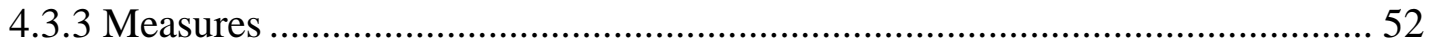

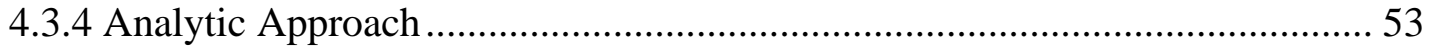

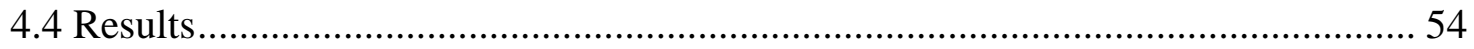

4.4.1 Summary Statistics .............................................................................. 54

4.4.2 Random Intercepts Regression of Organizational Characteristics .................. 56

4.4.3 Random Intercepts Regression of Processes of Care and Resident Population

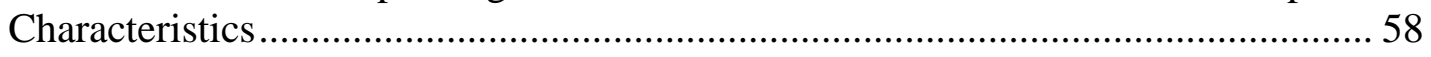

4.4.4 Fully Adjusted Random Intercepts Regression Model ...................................... 59

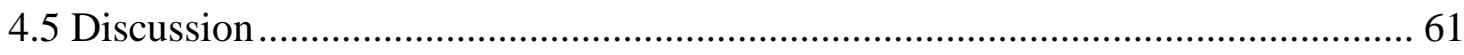

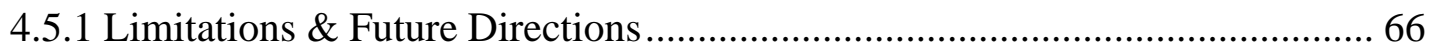

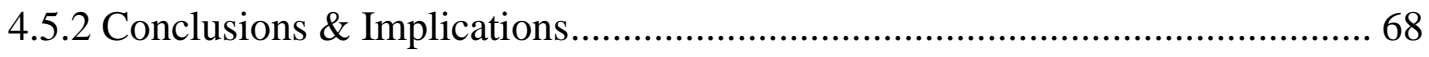

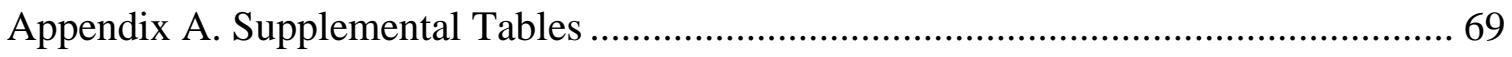

Chapter 5: Beyond the Medication Pass: Attitudes, Ethics, Agency, and Antipsychotic Medications in Assisted Living/Residential Care ............................................................ 72

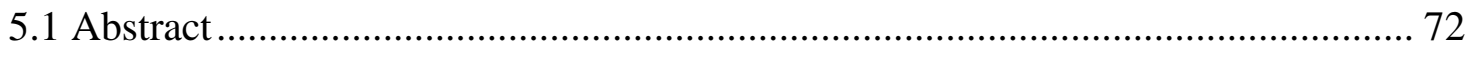

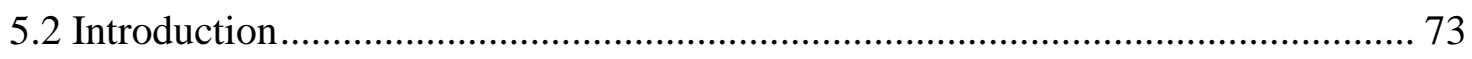

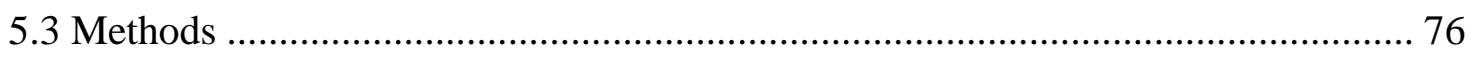

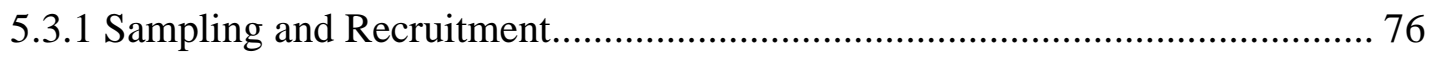

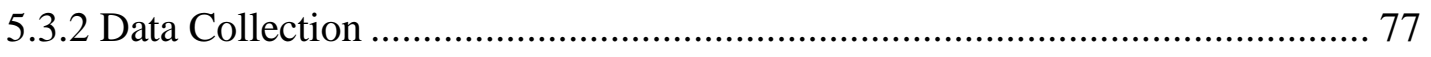




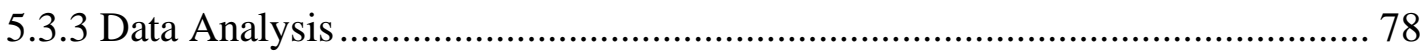

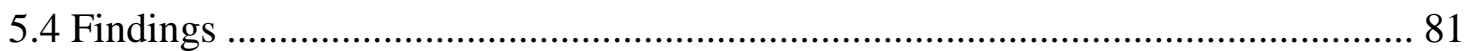

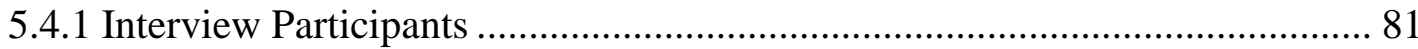

5.4.2 Theme 1: Positive, negative, and neutral beliefs and attitudes justify as-needed

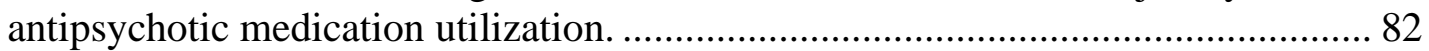

5.4.3 Theme 2: Responding to a resident's behavioral expression with or without medication is partially driven by an underlying morality/ethic........................... 85

5.4.4 Theme 3: Proximity to medication administration is inversely related to

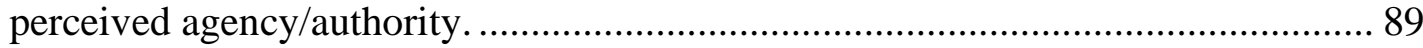

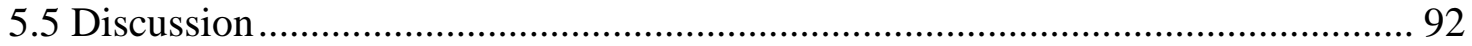

5.5.1 Limitations \& Future Directions ....................................................................... 96

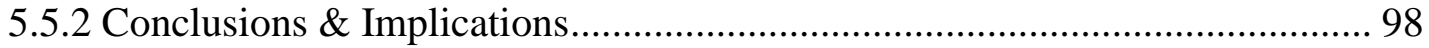

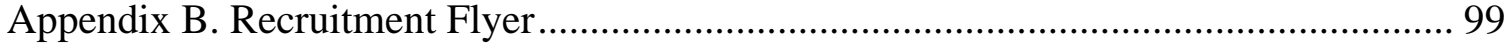

Appendix C. Text from Recruitment Questionnaire and Consent Form for Potential

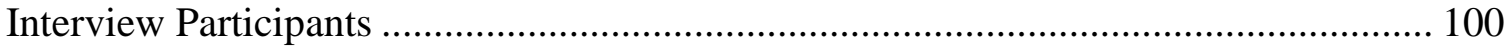

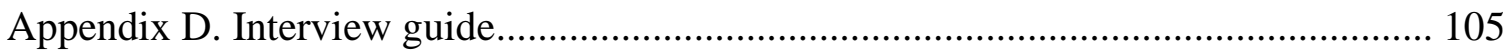

Appendix E. Ordered situational map of participant interviews ................................. 106

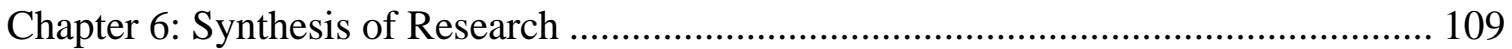

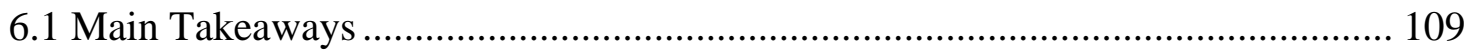

6.2 Social World of Antipsychotic Medication Use in AL/RC .............................. 112

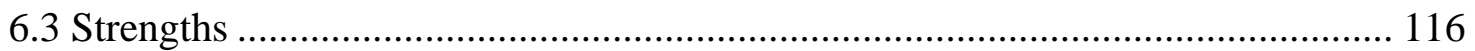

6.4 Limitations and Future Directions ............................................................. 117

6.5 Conclusion: Public Health Significance ........................................................... 119

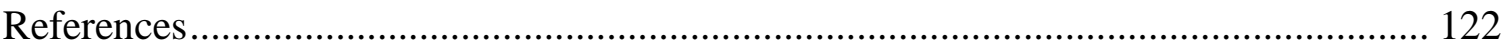




\title{
Chapter 2
}

\author{
List of Tables
}

Table 1. Dissertation stakeholder advisory committee.

\section{Chapter 4}

Table 1. Proportions, means, and standard deviations of responding assisted living/residential care and memory care setting characteristics, 2017-2019.

\section{$\underline{\text { Appendix A }}$}

Table 2. Bivariate random intercepts regressions of organizational, process, and resident population characteristics associated with prevalence of antipsychotic use by setting type, 2017-2019.

Table 3. Results of random intercepts regression of organizational, care processes, and resident population characteristics over prevalence of antipsychotic use, by setting type, 2017-2019.

Table 4. Results of fully adjusted random intercepts regression models of organizational, care processes, and resident population characteristics over prevalence of antipsychotic use by setting type, 2017-2019

\section{Chapter 5}

Table 1. Descriptive characteristics of interview participants 


\section{Chapter 2}

List of Figures

Figure 1. Multilevel nature of assisted living/residential care (AL/RC) settings.

Figure 2. Adapting the Institutional Analysis and Development framework to

antipsychotic medication use in assisted living/residential care settings.

Figure 3. Institutional analysis and development framework operationalized through situational analysis.

\section{Chapter 4}

Figure 1. Application of Donabedian's model to antipsychotic medication use in assisted living/residential care settings.

Figure 2. Results from random intercepts regression analysis of organizational characteristics and prevalence of antipsychotic medication use by setting type, 20172019

Figure 3. Results of random intercepts regression analysis of processes of care and resident population characteristics and prevalence of antipsychotic medication use by setting type, 2017-2019.

Figure 4. Results of random intercepts regression analysis of organizational, processes of care and resident population characteristics and prevalence of antipsychotic medication use by setting type, 2017-2019.

\section{Chapter 5}

Figure 1. An example of a messy map constructed of interviewee \#9. 80

Figure 2. Positional map of expressed ideologies on PRN antipsychotic medication use in assisted living/residential care residents with dementia........................... 82

Figure 3. Positional maps of perceived morality associated with response to assisted living/residential care residents' behavioral expressions ............................ 87

Figure 4. Positional map of perceived agency and proximity to PRN antipsychotic administration in assisted living/residential care settings.

\section{Chapter 6}

Figure 1. Social world of antipsychotic medication use in Oregon's assisted living/residential care settings 
List of Abbreviations

ADRD: Alzheimer's disease and related dementias

AHCA/NCAL: American Health Care Association/National Center for Assisted Living

AL: Assisted living

AL/RC: Assisted living/residential care

APU: Antipsychotic medication use

DHS/APD: Department of Human Services Office of Aging and People with Disabilities

LTC: Long-term care

LTSS: Long-term supports and services

MC: Memory care

NH: Nursing home

RC: Residential care

SNF: Skilled nursing facility

QMC: Quality Metrics Council 
Chapter 1. Introduction and Research Aims

Assisted living and residential care (AL/RC) settings serve about 812,000 older adults with significant care needs in the U.S. ${ }^{1}$ AL/RC settings provide assistance with care needs, including activities of daily living (ie, eating, dressing, bathing/showering, toileting, and mobility), chronic disease management, medications, behavioral expressions associated with dementia, and cognitive decline. An estimated $42 \%$ of $\mathrm{AL} / \mathrm{RC}$ residents have dementia, over $70 \%$ have cognitive impairment, and more than $75 \%$ receive assistance with medication administration. ${ }^{1,2}$ Most individuals living with Alzheimer's disease and related dementias (ADRD) or cognitive impairment experience behavioral expressions (eg, neuropsychiatric symptoms, behavioral and psychological disturbances), which have biological, psychological, and environmental underlying causes and can represent an attempt to communicate unmet needs. ${ }^{3-8}$

Behavioral expressions in persons living with ADRD are associated with family and paid caregiver burden and may motivate residential and care transitions. ${ }^{9-12}$ Though clinicians, dementia care experts, and researchers recommend nonpharmacologic (ie, non-medical) interventions to respond to behavioral expressions, individuals' distress may persist, which may warrant medical intervention. ${ }^{13-15}$ Psychotropic medications are often used to manage behavioral expressions, considered nonstandard, or "off-label" use. ${ }^{16-18}$ Antipsychotic medication, specifically in older adults with ADRD, has been associated with many adverse effects, including increased risk of falls, hospitalizations, and early mortality. ${ }^{19-23}$ Coupled with these findings and policy attention in nursing 
homes $(\mathrm{NH}),{ }^{24,25}$ reducing inappropriate antipsychotic medication use (APU) in older adults has been considered as a quality indicator in AL/RC settings. ${ }^{24,26-28}$

APU in AL/RC settings is an under-studied and controversial health policy issue. Clinicians, AL/RC providers, caregivers, pharmacists, and families differ in their beliefs on the efficacy and risks associated with APU in AL/RC residents, especially those with dementia. ${ }^{29-32} \mathrm{AL} / \mathrm{RC}$ regulations vary by state, so examining an individual state can provide important policy and practice contexts. It is crucial to investigate why antipsychotic medications are used and not used in AL/RC populations to understand the context-dependent administration of these medications beyond NHs.

This dissertation study employs the Institutional Analysis and Development (IAD) framework to analyze the situation of APU within AL/RC settings, acknowledging the dynamic and multilevel nature of this issue. ${ }^{33-37}$ For the research community, this study uses AL/RC setting-specific data to contextualize decision making and administration of antipsychotic medications. For policy makers, findings will inform the Oregon Department of Human Services/Aging and Persons with Disabilities' (DHS/APD) current and ongoing implementation of an antipsychotic medication quality metric, ${ }^{38}$ by providing state-specific evidence and context of antipsychotic medication use in AL/RC settings. For AL/RC providers and advocacy organizations, this research will summarize aspects of regulatory noncompliance with psychotropic medications, describe statewide patterns of APU, and center the perspectives of frontline care staff of $\mathrm{AL} / \mathrm{RC}$ residents in Oregon. For clinical practitioners, this research will use the experiences of $\mathrm{AL} / \mathrm{RC}$ setting staff and pharmacists to eventually develop a 
considerations fact sheet for the use of antipsychotic medications in their patients. The long-term objective of this research is to address controversial issues in medication management in AL/RC populations by developing a context-specific evidence base.

The proposed research is innovative because it embraces the complex, contextual nature of APU and decision-making in AL/RC settings. Most research on antipsychotic medication use in long-term care is conducted in NH populations. The American Health Care Association/National Center for Assisted Living (AHCA/NCAL) currently uses the quality metric defined by long-stay $\mathrm{NH}$ resident data (ie, reducing off-label APU to $15 \%$ ), which does not address appropriate/inappropriate administration and might not be relevant to $\mathrm{AL} / \mathrm{RC}$ settings. ${ }^{26}$ Due to state variation in $\mathrm{AL} / \mathrm{RC}$ licensing regulations and lack of representative longitudinal data, there is little evidence to inform an AL/RCspecific quality indicator for APU. It is crucial to understand APU beyond NH settings to avoid unintended policy consequences. ${ }^{27,28}$

The positions and identities I bring to this work inform my framing, analysis, and conclusions. Though I live with and receive both pharmaceutical and nonpharmaceutical treatment for mental illness as someone who currently lives independently, managing my health and social environments, my experience with the research topic at hand is limited to academic study. I have never worked as a paid caregiver for older persons living with ADRD, either at home or in a long-term care setting, and I am not an older adult living or receiving care in an $\mathrm{AL} / \mathrm{RC}$ setting. Understanding different perspectives regarding $\mathrm{APU}$ in AL/RC settings is a key goal of this study. Building on existing partnerships with local 
and state organizations, I rely on the experience and expertise of external stakeholders to guide and offer feedback on the implications of this study.

\subsection{Specific Aims}

Assisted living and residential care (AL/RC) settings serve approximately 812,000 older adults with significant care needs in the U.S. AL/RC settings assist with managing resident needs such as activities of daily living, chronic disease, health-related symptoms, medications, and behavioral symptoms associated with dementia.

Approximately $42 \%$ of residents have a dementia diagnosis, over $70 \%$ have cognitive impairment, and more than $75 \%$ receive assistance with medication administration. Most older adults with dementia and/or cognitive impairment exhibit behavioral symptoms that might attempt to communicate unmet needs. More than one-third of AL/RC residents exhibit such behaviors. These behavioral symptoms are associated with family and paid caregiver burden and may motivate a transition into or out of long-term residential care. Psychotropic medications (e.g., atypical antipsychotics, benzodiazepines) are often used to manage behaviors, which is considered nonstandard use. APU in older adults with dementia is associated with increased risks of falls, hospitalizations, and early mortality.

Antipsychotic medication administration in AL/RC settings is an under-studied and controversial health policy issue. Clinicians, AL/RC providers, caregivers, pharmacists, and families disagree on the efficacy and risks associated with antipsychotic medications in $\mathrm{AL} / \mathrm{RC}$ residents, especially those with dementia. AL/RC regulations vary by state, so examining an individual state can provide important policy and practice contexts. Oregon currently provides services to an estimated 24,000 AL/RC residents 
across 535 licensed settings, and decreasing APU in this population has been identified as

a public policy priority. ${ }^{38,39}$ To understand the context-dependent administration of these medications in settings other than nursing homes, it is crucial to understand why antipsychotic medications are used and not used in AL/RC populations.

The overall objective of this study is to inform the current debate about antipsychotic medication policies and practices in Oregon's AL/RC settings by using multiple data sources that represent licensing agency, setting, and care provider perspectives. To acknowledge the dynamic and multilevel nature of this issue, this study uses the Institutional Analysis and Development (IAD) framework to analyze the situation of antipsychotic medication administration. Understanding different points of view regarding antipsychotic medications is a key goal of this study. Thus, a stakeholdergroup will be formed building on existing partnerships with state organizations. I propose an integrated methods study to examine why antipsychotic medications are administered, or not, in AL/RC settings to achieve the following aims:

\section{Aim 1: Identify case-profiles of psychotropic medication noncompliance in}

Oregon AL/RC settings from 2014-2019. Method. Thematic analysis of public administrative data of psychotropic medication-related facility citations. I will use qualitative coding to develop case profiles of organizational noncompliance with state regulations, and to specifically understand the scope of noncompliance related to antipsychotic medications.

Aim 2: Examine whether structural characteristics are associated with prevalence of antipsychotic medication administration among Oregon AL/RC 
settings. Method. Random intercepts regression of four waves of the repeated crosssectional Community-Based Care (CBC) study (2015-2019), adjusting for resident population characteristics over time.

\section{Aim 3: Describe reasons for antipsychotic medication use and non-use in}

Oregon AL/RC settings. Method. I will sample four case communities based on findings from aims one and two. Within each case community, I will recruit staff to participate in "think aloud" interviews to respond to a resident behavior scenario or describing an opportunity for antipsychotic medication administration. Thematic analysis will be used to qualitatively compare responses from selected communities and staff types.

Impact: Currently, there is insufficient setting-specific evidence to set appropriate metrics to inform regulations that support the provision of care for AL/RC residents, especially with dementia. This research will provide multidisciplinary perspectives on antipsychotic medication use, non-use, and administration decision making in Oregon AL/RC settings for population-informed and evidence-based state policy development.

\subsection{Dissertating During a Global Pandemic}

I proposed the specific aims detailed in section 1.1 at the end of February 2020, just as worldwide alarms began to sound regarding the novel coronavirus (SARS-COV-2) pandemic. Nine days after the proposal defense for this dissertation (March 8, 2020), Oregon Governor Kate Brown issued the first state of emergency due to coronavirus outbreaks across the state. ${ }^{40}$ In Oregon, despite accounting for seven percent of total state cases (total $n=339,556), \mathrm{NH}$ and AL/RC residents comprised nearly half of total state 
deaths (total $n=3,959$ ) since the onset of the pandemic. ${ }^{41}$ The disproportionate impact of COVID-19 on these settings combined with emergency restrictions and disruption of inperson research activities through the Institutional Review Board inhibited me from implementing this study as proposed. The original study design relied on in-person, onsite, long-term data collection in collaboration with approximately four AL/RC communities in Oregon. Instead, I had to pivot to remote data collection. With guidance and support from my dissertation committee and external stakeholders, I transitioned the methods presented in Aim 3 from in-person shadowing of AL/RC staff to remote, semistructured interviews. This change, among other pandemic-associated barriers (eg, recruitment), significantly impacted my ability to implement this study as truly mixed methods and a complete situational analysis. I implemented each aim sequentially and individually, not truly integrating sampling design or analytic approaches. Chapter 2 provides a brief overview of the literature, theoretical, and methodological frameworks that inform this research study. Chapters 3 through 5 are the empirical articles that align with the aims proposed in section 1.1. Finally, Chapter 6 weaves together study findings, reflections, and policy/practice implications of this work. While not fully implemented as initially proposed, the spirit and intent of this dissertation study remain intact, offering several considerations for future gerontological researchers, long-term care providers and clinicians, and state policymakers. 
Chapter 2: Literature Review

The number of older adults (65+ years) in the United States (U.S.) will grow from 49 million to 95 million people by 2060 , with the fastest growth among the oldest old (85 years+) category. ${ }^{42}$ Older age is the most significant risk factor for developing Alzheimer's disease and related dementias (ADRD) and the sixth leading cause of death in the U.S. ${ }^{43,44}$ By 2050, it is estimated that almost 13 million adults over the age of 65 will be living with Alzheimer's disease and other related dementias (ADRD). ${ }^{44}$ Dementia is an overarching term for symptoms that accompany severe neurocognitive impairment that inhibits a person's ability to perform everyday activities such as dressing, bathing, and managing finances, commonly preceded by cognitive impairment. ${ }^{44,45}$ The cognitive decline that accompanies progressive symptoms classified as dementia greatly impacts a person's ability to live independently; individuals living with ADRD can experience personality changes, memory loss, behavioral changes, sleep changes, and ability to perform activities of daily living. ${ }^{5,8,46}$ In addition to the impacts on individual health and wellbeing that accompany the development of ADRD, there are also significant and multilevel psychosocial, financial, service provision, and healthcare system implications of an aging population with increasing incidence of ADRD. ${ }^{47-49}$ An estimated $70 \%$ of adults who live to the age of 65 will have significant longterm services and supports (LTSS) needs, with approximately one-third using these services for multiple years. ${ }^{50}$ Different LTSS options exist along a continuum, ranging from home health and personal care to skilled nursing facilities. ${ }^{51}$ This dissertation study 
focuses on one type of LTSS setting: assisted living and residential care (AL/RC), which is outpacing nursing homes $(\mathrm{NH})$ in terms of availability and utilization. ${ }^{52-54}$

As the population continues to age, adapting to and caring for the increasing proportion of those with dementia and cognitive impairment is a community health imperative. In this chapter, I briefly describe AL/RC as an LTSS option, behavioral expressions by and management of residents with ADRD in these settings, and the specific issue of antipsychotic medications as a response to behaviors associated with dementia. I ground these topics and issues within the Oregon state context.

\subsection{Assisted Living as a Long-Term Services and Supports Option}

Along the LTSS continuum, AL/RC settings are congregate environments where administrative, maintenance, and care staff coordinate care and provide services to residents. AL/RC settings provide housing, social support, medication management, and some health-related services to older adults and disabilities. Unlike NH, AL/RC settings are not licensed health facilities and typically rely on paraprofessional, or "unlicensed," direct care staff. These settings do not require direct care workers to have healthcertifications such as a certified nursing assistant, licensed professional or vocational nurse, or medication technician certifications. Individual states regulate assisted living settings, meaning there are regulatory, operational, and resident population variations. Settings are meant to have enough staff scheduled to provide care based on the needs of their resident populations. AL/RC settings are meant to serve individuals who need assistance with activities of daily living, medication management, household management, and social support. 
In the U.S., nearly 29,000 licensed AL settings provided care to approximately 812,000 residents. ${ }^{1}$ Over $40 \%$ of these residents have a dementia diagnosis and more than $70 \%$ have some level of cognitive impairment. ${ }^{1,2}$ However, the true prevalence of cognitive impairment and dementia among older adults are likely higher than reported, given the under diagnosis of both conditions. ${ }^{46,55,56}$

\subsection{Behavioral Expressions in Older Adults with Dementia or Cognitive Impairment}

"Neuropsychiatric symptoms," "behaviors," "behavioral expressions," and "behavioral and psychological symptoms of dementia" (hereafter, behavioral expressions) describe the behavioral and affective expressions of both dementia and cognitive impairment. These include aggression, agitation, anxiety, delusions, hallucinations, and sleeplessness. ${ }^{5,7,8,57-60}$ Nearly all older adults with dementia and/or cognitive impairment experience at least one behavioral expression. , $6,60^{-6}$

Current assessment of behavioral expressions, for clinical or research purposes, typically capture behavioral events either during or after occurrence and rely on proxy descriptions of the events. ${ }^{61}$ Conceptualization and understanding of behavioral expressions and the antecedents leading to their occurrence influence the response or treatment. Historically, the geriatric field categorized behavioral expressions as something purely biological resulting from neurocognitive decline. ${ }^{5}$ More recently, clinicians and researchers posit that these behavioral expressions have multiple, diverse causes, including neurobiological disruptions in the central nervous system, pain, unmet needs, pre-existing personality/psychiatric conditions, environmental infrastructure, or over/under stimulation. ${ }^{3-5,62,63}$ Behavioral expressions can represent an individual's effort 
to communicate unmet needs such as sensory deprivation, loneliness, physical pain, activities of daily living, or meaningful activities. , $^{3,64,65}$

It is estimated that more than $34 \%$ to $38 \%$ of $\mathrm{AL} / \mathrm{RC}$ residents exhibit behavioral expressions..$^{2,66}$ One study found that over $70 \%$ of AL/RC residents with a diagnosis of dementia or cognitive impairment experience behavioral expressions. ${ }^{2}$ A more recent investigation discovered that of $\mathrm{AL} / \mathrm{RC}$ residents who do express behaviors, staff only documented what caused the behaviors in about one quarter of cases. ${ }^{10}$ Chronic or severe behavioral expressions have implications for quality of life, family and caregiver burden, and care transitions. ${ }^{11,12,67}$ One study found that behavioral expressions were not significantly associated with self-assessed quality of life of individuals who experienced them, but there was a significant association with caregiver assessment of their quality of life. ${ }^{68}$ These behaviors are associated with increased family and paid caregiver burden and may motivate a transition from home to a community-based care setting, or to an emergency department from long-term care. ${ }^{11,69-71}$

\subsection{Responding to Behavioral Expressions Associated with Dementia or Cognitive Impairment in Older Adults}

There are two overarching approaches to managing behavioral expressions: nonpharmacologic and pharmaceutical. Nonpharmacologic interventions are behavioral, psychosocial, or environmental in nature while pharmaceutical interventions are medications used to treat or manage behavioral expressions. Existing guidelines and practices encourage psychosocial or environmental interventions as the first line of treatment in managing behavioral expressions as a person-centered, safe, alternative to 
medication. ${ }^{15,72,73}$ Nonpharmacologic interventions include sensory, cognitive, and environmental therapies that can be used to prevent the onset of an individual's behavioral expression (eg, aggression, restlessness, wandering) or to mitigate the behavioral expression. ${ }^{15}$ These range from care practices to explicit protocols and therapies. Person-centered care practices such as providing meaningful activities, incorporating individuals' preferences, and improving relationships between care providers and individuals with dementia have been associated with decreased agitation, depression, and increased quality of life. ${ }^{73-76}$ Nonpharmacologic therapies to mitigate behavioral expressions include but are not limited to, aromatherapy, multisensory therapy, reminiscence therapy, music therapy, validation therapy., ${ }^{4,15}$

When nonpharmacologic interventions fail or a resident is in considerable distress, caregivers may try pharmaceutical management of behaviors using psychotropic medication. ${ }^{77,78}$ Psychotropic medications interact with the central nervous system and include the following drug classes: antipsychotics, antidepressants, anxiolytics, hypnotics, and mood stabilizers. ${ }^{79,80}$ Clinical guidelines. randomized control trials, and observational studies suggest the use psychotropic medications in older adults, particularly antipsychotic medications in those with dementia or cognitive impairment, are associated with greater risks than benefits. ${ }^{20,81-85}$ Specifically, antipsychotic medication use in older adults with dementia has been associated with an increased risk of potential medication-related side effects, ${ }^{86}$ falls, ${ }^{87}$ hospitalizations,${ }^{23,88}$ and early mortality..$^{19,21,22,89-91}$ Understanding why antipsychotic medications are used among older adults with dementia is a safety, quality of life, and public health issue..$^{92}$ 


\subsection{Antipsychotic Medications Use Disparity in Older Adults with Dementia}

Antipsychotic medication use (APU; prescription and administration) in AL/RC settings is an under-studied and controversial health policy issue. In response to findings regarding the risks associated with APU in older adults with dementia, the U.S. Food and Drug Administration first issued a boxed ("black box") warning for APU in older adults in 2005, which has been associated with lower antipsychotic use among older adults with dementia. ${ }^{93-95}$ The Alzheimer's Association best practices for dementia care recommends the use of antipsychotic medications in individuals with dementia only after attempting a nonpharmacologic approach and under the following conditions: 1) behavioral symptoms due to psychosis, 2) symptoms put an individual or others in danger, or 3) experiences of inconsolable, persistent distress or substantial difficulty receiving needed care. ${ }^{13,15,96}$ However, antipsychotic medications are prescribed for conditions outside of approved indications, or "off-label," to manage behavioral expressions. ${ }^{17,18,97,98}$ Overall, AL/RC administrators, direct care and nursing staff, and families of AL/RC residents reportedly view the use of these medications positively and effectively. ${ }^{29-31,99-101}$ Despite current initiatives that focus on reducing antipsychotic use in the $\mathrm{NH}$ population, ${ }^{25,102-104}$ there is still lack of AL/RC-specific evidence regarding how APU, staff training and implementation of nonpharmacologic interventions, uptake of other psychotropic medications beyond antipsychotics, or potential discrimination against older adults whose behaviors are deemed "challenging." ${ }^{12,63,105-109}$ The current debate regarding when or if to use antipsychotic medications to respond to behavioral expressions creates a complex 
situation with multidisciplinary actors that is difficult to solve with "one-size fits all" policies. $^{27,110}$

\subsection{Assisted Living/Residential Care Context in Oregon}

AL/RC settings are licensed and regulated by individual states; examining a single state can provide important policy and practice contexts. ${ }^{111-114}$ Oregon is considered a regulatory and care provision pioneer of modern day AL/RC in the U.S. ${ }^{115,116}$ In Oregon, an estimated $18.2 \%$ of the population $(767,628$ people) are 65 years or older. ${ }^{117}$ Oregon's older adult population is anticipated to reach more than $20 \%$ by $2030 .{ }^{118}$ A recent study estimated that $70 \%$ of adults who age into older adulthood (65 years or older) will develop significant LTSS needs and nearly half will receive some form of paid care over the course of their older adult years. ${ }^{50}$ In the last decade, the number of AL/RC settings has increased while the number of $\mathrm{NH}$ settings has

decreased. ${ }^{53,54,119}$ AL/RC settings in Oregon have increased by 9.4\% overall (489 in 2014 to 535 in 2019), with a $30 \%$ increase in settings endorsed to provide memory care (148 in 2014 to 193 in 2019). ${ }^{120,121}$

AL/RC setting types in Oregon include assisted living (AL), residential care (RC), and memory care (MC) communities. RC settings have different physical environments from AL but operate under the same regulations. ${ }^{122} \mathrm{MC}$ communities can either be standalone buildings, or units within AL/RC endorsed to provide ADRD-specific care. ${ }^{123}$ Oregon collects longitudinal data on the AL/RC population including setting-level prevalence of APU. Over 24,000 Oregon residents currently receive supportive health and housing services within AL/RC settings in Oregon. ${ }^{121}$ This state study shows that 
$47 \%$ of current residents have a dementia diagnosis, and $27 \%$ took an antipsychotic medication in the prior 30 days. However, these rates differ by setting type: $17 \%$ of AL, $30 \%$ of RC, and $44 \%$ of MC residents reportedly received an antipsychotic medication in the prior 30 days. ${ }^{121}$ These estimates are higher than APU rates reported by NH in Oregon (15\%) $)^{124}$ and nationally (14\%). ${ }^{104}$

In observational studies of $\mathrm{NH}$, both resident (eg, gender, age, behavioral expressions, dementia diagnosis, Medicaid coverage) and setting (eg, profit status, geography, occupancy rate, staff mix) characteristics have been correlated with higher APU rates. ${ }^{125-128}$ Studies suggest pain and behavioral expressions of long-term care residents with ADRD remain underrecognized and undertreated. ${ }^{129-131}$ Few studies that have examined $\mathrm{AL} / \mathrm{RC}$ setting and resident characteristics associated with antipsychotic use found mixed evidence that setting size, geography, resident age, gender, ADRD and/or psychiatric diagnosis, and expression of behavioral symptoms were associated with APU. ${ }^{23,132,133}$ A cross-sectional study of Oregon AL/RC residents found that residents living in MC settings, expressed more behaviors, and had a diagnosis of ADRD were more likely to receive an antipsychotic medication in the last week compared to their counterparts, and residents living in nonprofit settings were less likely to receive an antipsychotic medication. ${ }^{134}$ However, associations among organizational and resident characteristics and rates of APU have not yet been explored at the population-level (ie, state). AL/RC settings need guidelines that respond to the context of the organizational environment and changes in the resident population that are not copied from $\mathrm{NH}$ research and policies. 
APU in AL/RC settings is a public policy priority in Oregon from a safety, oversight, and quality perspective and involving multidisciplinary actors evidenced by updates to licensing regulations and recent legislation focused on this topic. Oregon Administrative Rules Chapter 411 Division 54 (OAR 411-054-0000) govern licensing, quality, and oversight of AL/RC settings. ${ }^{122}$ OAR 411-054-0055-6 addresses the use of psychotropic medications, including antipsychotics, in AL/RC residents. Specifically, $\mathrm{AL} / \mathrm{RC}$ settings may only use these medications to treat a resident's medical symptoms or maximize functioning; cannot request psychoactive medications to treat behavioral symptoms without clinical consultation; and must document initial non-pharmacologic interventions before administering psychotropic medications on an as-needed basis.

State surveyors conduct inspections, interview staff and residents, and review resident records to assess regulatory compliance at least once every 24 months for $\mathrm{AL} / \mathrm{RC}$ settings. AL/RC settings may be cited for regulatory noncompliance, determined based on the severity and scope of the violation. Medication treatment orders (C303), medication administration (C310), and psychotropic medications (C330) are consistently ranked in the top fifteen most frequent citations for noncompliance among Oregon AL/RC settings. ${ }^{135}$

In the 2017 legislative session, Oregon passed two bills regarding quality of care in AL/RC settings. House Bill 3359 introduces additional quality oversight, such as developing the Quality Metrics Council (QMC) comprising state policy makers, AL/RC representatives, clinicians, and academic experts, and requiring AL/RC settings to have policies and procedures in place to reduce medication errors. ${ }^{136}$ House Bill 3262 
introduces additional oversight intended to reduce adverse side effects and nonstandard long-term APU in older adults and persons with disabilities in long-term care. ${ }^{39}$ However, some QMC members have raised concerns that the goal of reducing APU will result in unintended consequences that may harm individuals with serious mental illness, or result in residents with behavioral expressions being transferred out of AL/RC settings. ${ }^{137,138}$

Table 1. Dissertation stakeholder advisory committee

\begin{tabular}{|c|c|c|}
\hline Name & Organization & Position \\
\hline Lynette Alvarado & $\begin{array}{l}\text { Concepts in Community } \\
\text { Living }\end{array}$ & $\begin{array}{l}\text { Regional Director of } \\
\text { Operations }\end{array}$ \\
\hline $\begin{array}{l}\text { Linda Bifano, DNP, RN, } \\
\text { MPA }\end{array}$ & Bifano Consulting, LLC & $\begin{array}{l}\text { Community-Based Care } \\
\text { Services Consultant }\end{array}$ \\
\hline Lindsey Bretzman & Mary's Woods & $\begin{array}{l}\text { Dementia Life Enrichment } \\
\text { Specialist }\end{array}$ \\
\hline Nirmala Dhar, LCSW & Oregon Health Authority & $\begin{array}{l}\text { Statewide Older Adult } \\
\text { Behavioral Health } \\
\text { Director }\end{array}$ \\
\hline Mauro Hernandez, PhD & ita Partners & Principal \\
\hline Linda Kirschbaum & $\begin{array}{l}\text { Oregon Health Care } \\
\text { Association }\end{array}$ & $\begin{array}{l}\text { Senior Vice President of } \\
\text { Quality Services }\end{array}$ \\
\hline Nancy Koerner, RN & Mary's Woods Retirement & $\begin{array}{l}\text { Vice President of Health } \\
\text { Services }\end{array}$ \\
\hline $\begin{array}{l}\text { Maureen Nash, MD, MS, } \\
\text { FAPA }\end{array}$ & Providence Elderplace & $\begin{array}{l}\text { Geriatrician Psychiatrist, } \\
\text { Medical Director }\end{array}$ \\
\hline
\end{tabular}

Stakeholder engagement and collaboration is critical to achieve the long-term objective of this research due to their contributions to the development of and familiarity with Oregon's sociohistorical AL/RC policy and practice context. Representatives from 
key organizations including the Oregon Department of Human Services/Aging and Persons with Disabilities (DHS/APD), Oregon Health Care Association (OHCA), Quality Metrics Council (QMC), LeadingAge Oregon, Oregon Partnership for Quality Dementia Care, and AL/RC providers will be asked to review and comment on data collection tools, to provide their organization's policies regarding APU, and respond to draft findings. I have recruited representatives of these organizations to form a stakeholder advisory committee (see Table 1). This committee will serve in part to establish research priorities, dissemination, and future directions for this work.

\subsection{Medication Management in AL/RC Settings}

Medication management in AL/RC settings is a complex process involving residents, direct care staff, nurses, prescribers, pharmacists, and administrators. ${ }^{139-144}$ As with all other operations in AL/RC settings, administrators are responsible for "ensuring adequate professional oversight of the medication treatment administration system" (OAR 411-054-0055-1a). ${ }^{122}$ Medication administration in Oregon AL/RC settings must also be reviewed quarterly by registered nurses or pharmacists (OAR 411-054-0055-1i). Direct care staff (eg, caregivers, certified nursing assistants, and medication aides) build and maintain relationships with their residents, learning from and basing their care decisions on residents' cognitive, physical, and emotional cues on a daily basis. ${ }^{141,145,146}$ In Oregon, direct care staff can assist with medication administration if supervised by a nurse. ${ }^{140,147}$ For those staff that assist medications administered "as-needed," knowing individual residents' behaviors and nuances is critical to decision making around administration. ${ }^{141}$ Direct care staff may view behavioral and psychological expressions of 
dementia negatively, ${ }^{12}$ and have several strategies to address these behaviors, such redirection, isolation, seeking other coworkers' assistance, or communication with external care providers. ${ }^{148}$ However, little is known about direct care staff and nurse's decision making and task-related responses to these behaviors. Few studies have examined how and why antipsychotic medications are used in AL/RC settings by incorporating multidisciplinary perspectives and data sources.

\subsection{Institutional Analysis and Development (IAD) Framework}

Institutions describe situations of human actions structured through rules, norms, and shared strategies. ${ }^{33,149}$ This "grammar of institutions"149 is the foundation of the Institutional Analysis and Development (IAD) framework, which identifies the key variables and components, including environmental and community characteristics and rules or norms, which contribute to an "action situation." $35,36,150,151$ An action situation consists of actors that occupy certain positions and perform actions relative to other actors involved (Figure 1). ${ }^{36,151}$ These positions and actions are also influenced by external variables, including available information, actors' level of control within the situation, and costs and benefits associated with actions taken, resulting in various interactions and outcomes. ${ }^{36,37}$

AL/RC settings are organizational contexts where residents, their families, direct care staff, administrators, clinicians, regulatory entities and other nonhuman elements (eg, service plans, resident records, treatment orders) interact through social and care relationships (Figure 1). ${ }^{152-154}$ At each contextual level shown in Figure 1 are different sets of actors, choices, positions, available information and influence, thus constituting 
different levels of analysis: the action situation (operational), authoritative decision making (collective choice), and the mechanisms by which collective choice is enacted (constitutional). ${ }^{35-37,150}$

Figure 1. Multilevel nature of assisted living/residential care (AL/RC) settings.

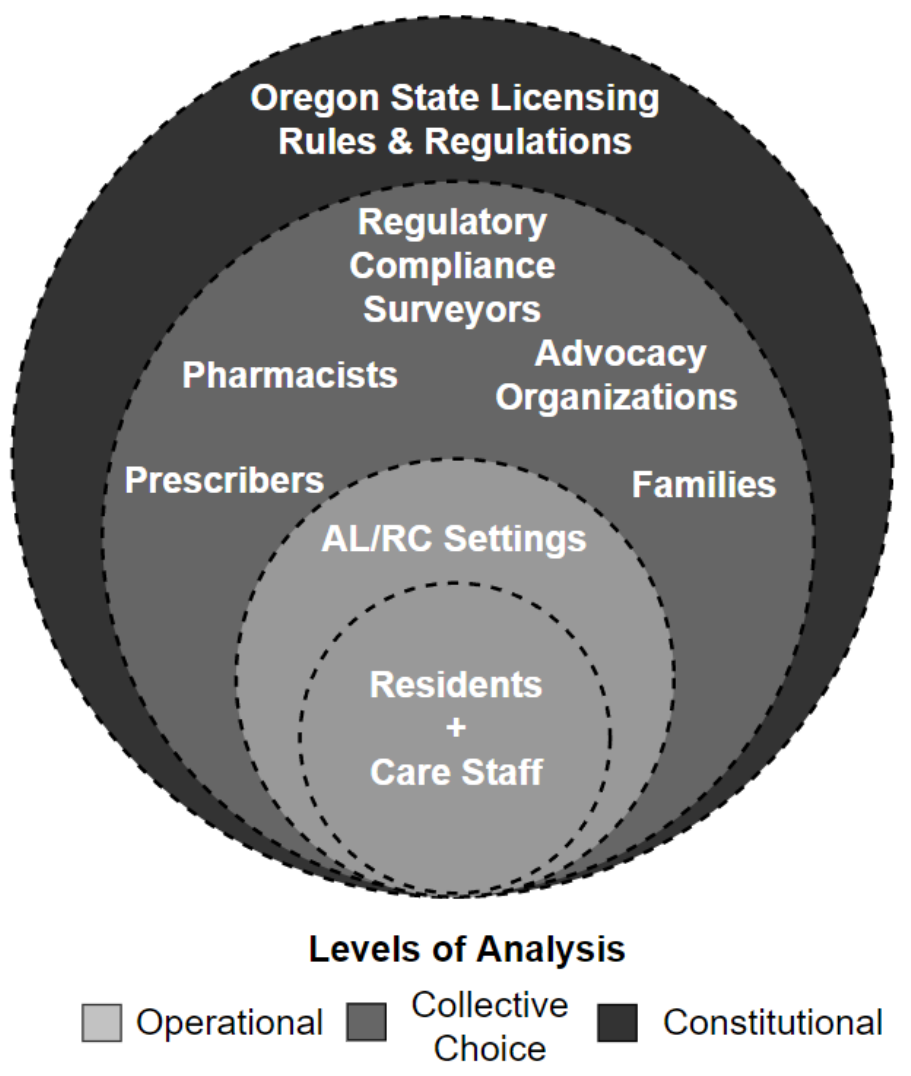

As conceptualized through the IAD framework, I position the AL/RC setting of care as an "institution" that frames the action situation of APU. Figure 2 maps the study elements of this dissertation onto an adaptation of Ostrom's conceptual model of the IAD framework. ${ }^{36}$ 
Figure 2. Adapting the Institutional Analysis and Development framework to antipsychotic medication use in assisted living/residential care settings.

\section{Exogenous Variables}

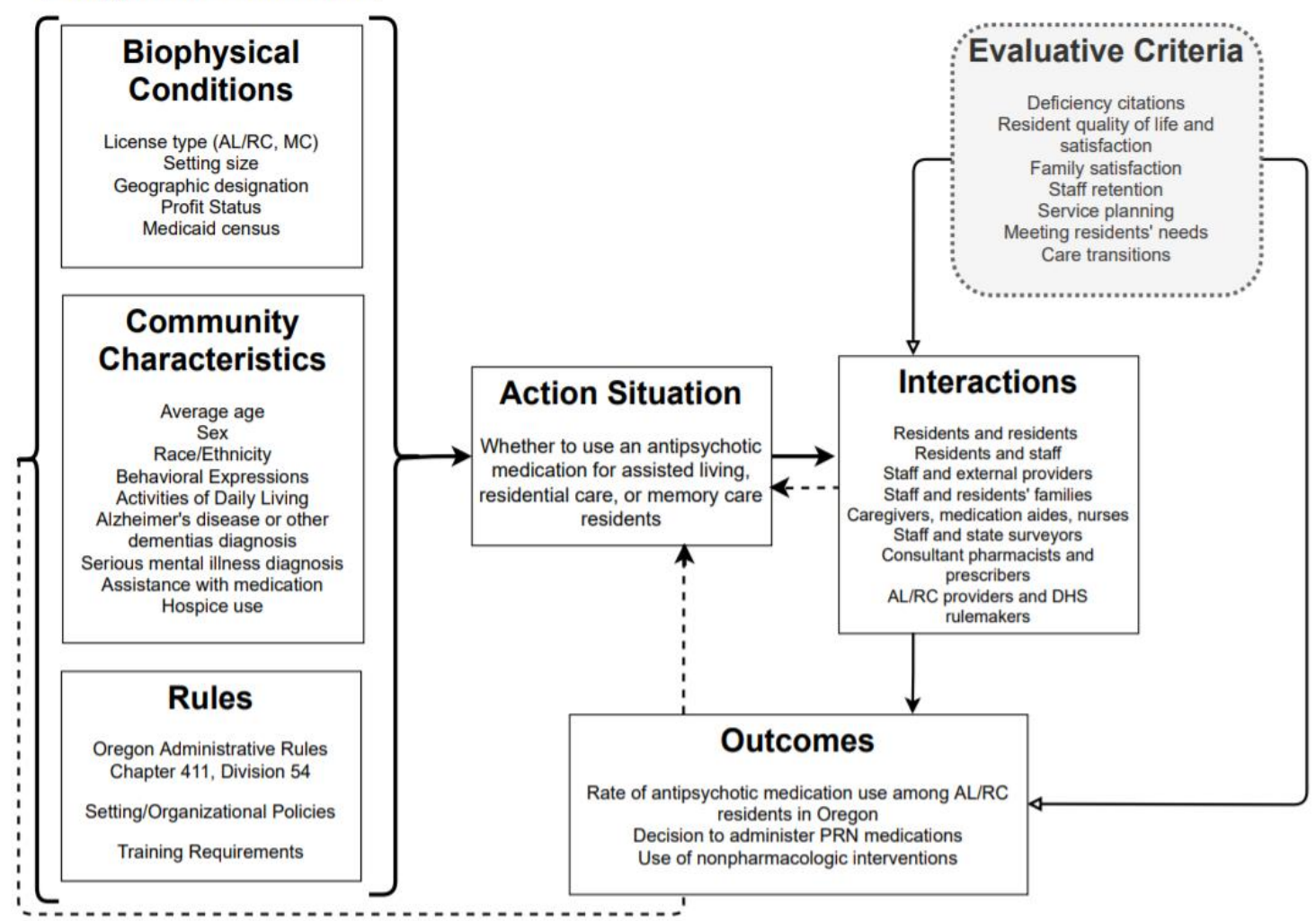

Note. Adapted from E. Ostrom. Background on the institutional analysis and development framework. Policy Stud. 2011;39(1):10.

The IAD framework highlights the multilevel and contextual nature of the overall dissertation study and assists with identifying "the elements and general relationships among these elements to consider for institutional analysis and organize diagnostic and prescriptive inquiry." ${ }^{151}$ In this study, I am interested in analyzing APU within AL/RC settings as an action situation. Actors involved include residents, direct care staff, medication aides, administrators, clinical providers, therapists, pharmacists, prescribers, state surveyors, and state policymakers. To understand the how and why behind APU in AL/RC settings, I use this framework to identify the other elements of this action 
situation: types of information, level of control, costs/benefit in decision making, positionality, and interactions among human, organizational, and material actors.

The IAD framework was formulated and adapted to study policy processes and socioecological systems, primarily used at the intersections of policy, governance, economy, and ecology, where external variables that influences an action situation (ie, biophysical conditions and community characteristics) have largely been conceptualized as elements of political or ecological environments. ${ }^{155-157}$ I have adapted the biophysical conditions described in the original framework to focus on material and structural conditions of $\mathrm{AL} / \mathrm{RC}$ settings, such as geographical designation (ie, urban and rural), setting size, and license type (ie, $\mathrm{AL} / \mathrm{RC}$ or $\mathrm{MC}$ endorsement). Additionally, community characteristics in the original framework describe the social and cultural context where an action situation takes place. ${ }^{37}$ I operationalize community characteristics with measures that describe the resident population within any one AL/RC setting (eg, proportion of residents with ADRD, proportion of residents with behavioral expressions). These measures only partly frame the situation of APU but provide context of the resident population within settings. Finally, the rules and norms that structure an action situation include the rules and regulatory parameters outlined through state licensure and staff training requirements.

These external variables influence what occurs within an action situation, resulting in outcomes. For this study primary outcomes of interest include the rate of APU in Oregon AL/RC residents and decision points regarding whether to administer antipsychotic medications. Interactions among the actors involved in the situation also 
play a role in determining the actions that take place and affects outcomes. Evaluative criteria are used by actors within the situation and/or observers of the situation, such as a doctoral student researcher, to identify areas of improvement and mechanisms that work well..$^{36,37}$

\subsection{Situational Analysis: Examining Social Worlds}

In section 1.1, I briefly describe the research methods associated with each of the three proposed aims of this dissertation study. In Chapters 3-5, I detail the specifics of the methods conducted to achieve these study aims. This section defines and describe the overarching philosophy and research methodology guiding the entire dissertation study: pragmatism and situational analysis. Briefly, pragmatism is a philosophy of meaning making that frames my approach to forming and pursuing the answer to the research question: How and why are antipsychotic medications used in AL/RC settings? Pragmatism emphasizes the pursuit of actionable knowledge (eg, applied knowledge), recognizing that knowledge is situated in prior experience and beliefs that inform action, and that multiple realities or truths exist based on this situated knowledge. ${ }^{158-162} \mathrm{~A}$ foundational element to this dissertation study is understanding how beliefs, actions, and situated knowledge inform APU in AL/RC residents by critically evaluating multiple data sources, perspectives of different actors, and considering the multilevel contexts of AL/RC settings. ${ }^{159}$

Pragmatism guides my approach to situational analysis, which I use to weave together findings across the three proposed research aims to understand APU in AL/RC settings in Oregon. Clarke developed situational analysis as an extension of grounded 
theory methodology. ${ }^{163}$ Grounded theory processes comprise integrated, iterative qualitative data collection and analysis, extensive reflexivity through analytic memos, and categorization of concepts to generate theory derived from - grounded - within the data. ${ }^{160,164-166}$ Grounded theory methods generally involve processes of coding, memoing, and theorizing over the course of data collection, where the researcher contemplates and interprets the patterns, topics, and concepts arising within the data. ${ }^{160,166}$

Situational analysis builds on the Straussian conceptualization of social worlds and negotiations. ${ }^{163,167-170}$ Strauss theorizes social worlds as universes of response, organization, and communication that can exist at any scale and subsist of limitless different subworlds, or segments. ${ }^{167,170}$ Building on prior work of Carder, the social world under investigation in this dissertation is the AL/RC setting. ${ }^{171,172}$ The segment, or situation, of interest is APU, which consists of human (eg, staff, residents, prescribers) and nonhuman (eg, antipsychotic medication, training, medication records) actors that interact together to negotiate social processes around medication administration. ${ }^{172}$ Situational analysis extends grounded theory by providing a method to identify, conceptualize, analyze, and visualize the situations which construct the processes that occur within a social world. ${ }^{163,166,173-177}$

As described in section 2.7, APU within AL/RC settings is the action situation of interest for this study, therefore, the unit of analysis. In addition to coding procedures and identifying elements within data, situational analysis consists of various data mapping: situational maps, social worlds maps, relational maps, and positional maps. ${ }^{173,174,176}$ Situational maps identify all the of the relevant elements of the action situation: human, 
nonhuman, ideological, discursive, symbolic, cultural, historical, temporal, etc. ${ }^{163,173,175}$ I provide two examples of situational maps, one in Chapter 5 pertaining to my third research aim and in Chapter 6 attempting to comprehensively describe APU in AL/RC across the entire dissertation study. One can use social worlds maps to draw out the actors (human and nonhuman) pertinent to a situation of interest and where they orient to the situation of interest to provide an understanding of the overarching discourses, negotiations, and interactions that take place. ${ }^{163,175}$ For example, AL/RC residents and care staff may operate within the arena of an AL/RC setting. Similarly, pharmacists, clinicians, and prescribers primarily perform within the context of the healthcare system (ie, clinics, offices). Finally, state legislators operate in the realm of regulations and policy construction. Relational maps attempt to make connections among the relevant elements identified in a situational map and positional maps plot the major positions identified regarding the situation of interest.

While the IAD framework acknowledges the dynamic nature of processes at a given contextual level through the feedback loop from outcomes to both external characteristics and the action situation (Figure 2), the action situation itself is linear (eg, actors $\rightarrow$ positions $\rightarrow$ information $\rightarrow$ decision $\rightarrow$ outcome). By utilizing the components of an action situation identified by the IAD framework and previous research on antipsychotic medication administration in this setting, an informed grounded theory approach will guide my descriptions and composition of the action situation. 
Figure 3. Institutional analysis and development framework operationalized through situational analysis.

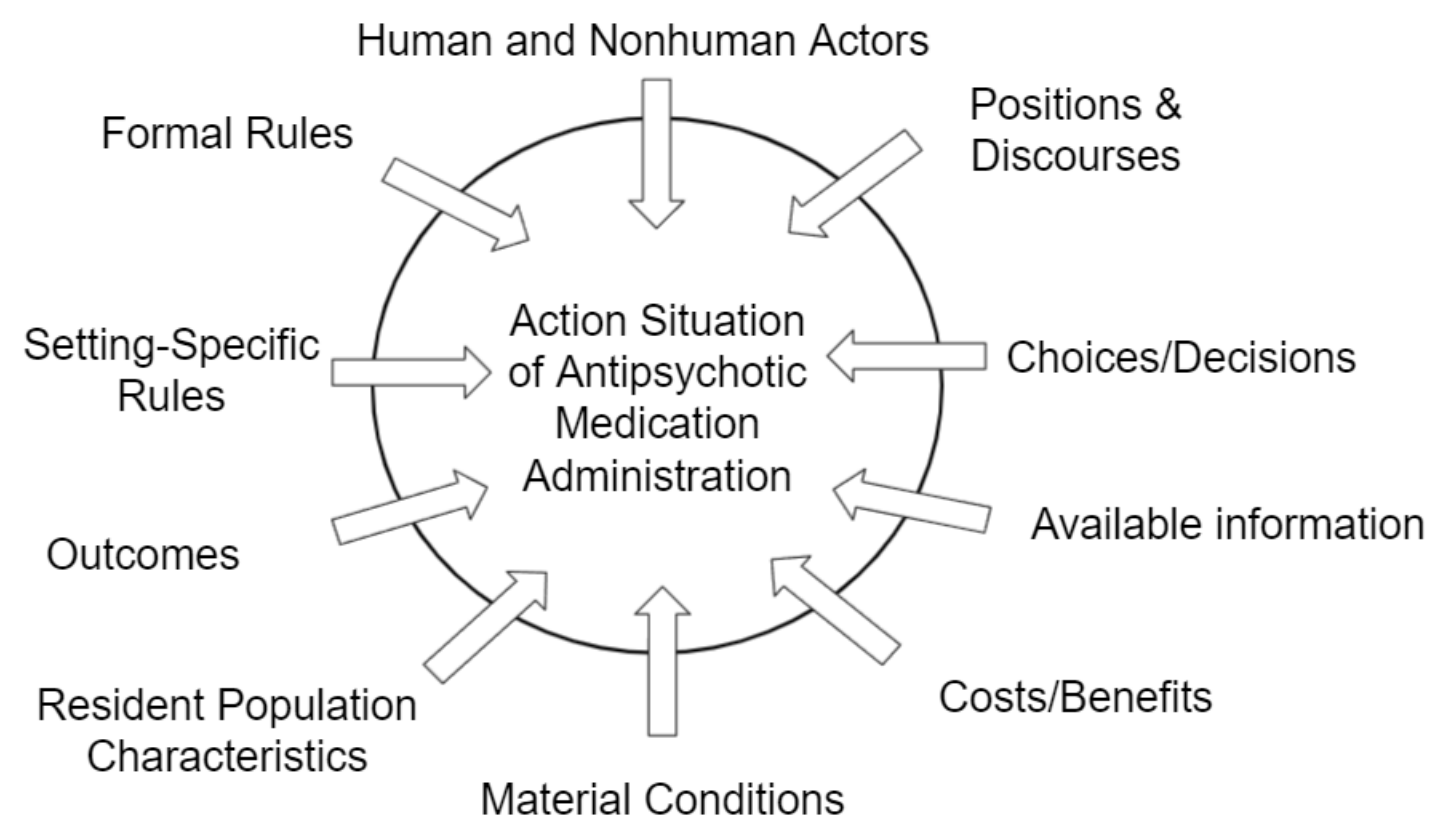

Situational analysis provides an approach to operationalize the IAD framework in the context of this study (Figure 3). Both Ostrom's framework and Clarke's methodological application work with the concept of an action situation, embracing complexity and context to explain and situate various phenomena. The research question and action situation influence the type of mapping used. In addition to the methods used to achieve the aims proposed in Chapter 1, I use situational analysis to draw conclusions across the overall dissertation study. 
Chapter 3: Paperwork, Paradox, and Pro Re Nata: Psychotropic Medication Deficiencies in Assisted Living/Residential Care

\subsection{Abstract}

Background: A significant proportion of assisted living/residential care (AL/RC) residents live with Alzheimer's disease or related dementias (ADRD) or cognitive impairment, conditions associated with behavioral expressions that may or may not be treated with psychotropic medications. Individual state approaches to AL/RC licensing and oversight in the United States result in different practice standards and requirements, including psychotropic medication use.

Methods: We use applied thematic analysis to examine the scope of 170 psychotropic medication deficiency citations issued in Oregon AL/RC settings from 2015-2019.

Results: We found most deficiency citations were issued for documentation errors regarding nonpharmacologic approaches and residents' behavioral expressions. Other themes include direct care workers' role paradox, lack of clinical consultation before requesting psychotropic medication order changes, and organization-level correction to prevent future deficiencies.

Discussion and Implications: Policymakers might consider how regulations unintentionally incentivize task-oriented versus person-centered care practices and incorporate $\mathrm{AL} / \mathrm{RC}$ provider experience during policy development. 


\subsection{Introduction}

In the United States, approximately 29,000 assisted living/residential care (hereafter 'AL/RC') settings provide a community-based long-term care option to over 800,000 residents. ${ }^{1}$ A significant share of AL/RC residents have an Alzheimer's disease or related dementia (ADRD) diagnosis (42\%), and an estimated $26 \%$ to $90 \%$ have cognitive impairment. ${ }^{1,2}$ Psychotropic medication used to manage behaviors associated with ADRD in long-term care including $\mathrm{AL} / \mathrm{RC}$ residents, ${ }^{103,133,178}$ presents a significant health policy concern because of their associations with adverse events and implications for care provision. ${ }^{25,110}$

Psychotropic medications - antipsychotic, antianxiety, antidepressant, sedatives, and hypnotics - are commonly used to respond to behavioral expressions in people living with ADRD. ${ }^{16,18,78}$ If nonpharmacologic (eg, psychosocial) interventions do not alleviate an individual's distress, medication may be used. ${ }^{77,96,179,180}$ The appropriateness of psychotropic medications to manage behavioral expressions associated with ADRD and cognitive impairment has received national and international attention. ${ }^{178,181-183}$

\subsubsection{Psychotropic Medication Deficiency Citations: What Can We Learn?}

In skilled nursing facilities, national regulatory standards and resident-level data collection have led to the development of national quality metrics (eg, Nursing Home Compare); the pursuit of quality can theoretically lead to better care processes and outcomes. ${ }^{184,185}$ When defining "quality," long-term care settings include deficiency citations, or measures of organizational noncompliance with regulations. ${ }^{186-188}$ Deficiencies have been associated with quality of the physical environment, staff 
turnover, resident safety, and resident-centered care. ${ }^{189-191}$ Compared to other types of medication-related citations, the prevalence of psychotropic deficiencies is low and mainly consists of documentation errors. ${ }^{192,193}$. Less is known about associations of AL/RC deficiency citations with resident outcomes, though existing research suggests most deficiencies do not pose severe risk to residents' health and safety, and that most medication errors are related to documentation. ${ }^{188,194-196}$

Individual states oversee $\mathrm{AL} / \mathrm{RC}$ regulations resulting in wide within and between state variations of licensing standards, staff training requirements, and admission/discharge criteria, limiting the utility of measuring and comparing quality of facilities across states. ${ }^{11,114,140,197}$ States, including Oregon, conduct periodic surveys of settings to evaluate regulatory compliance, issuing surveys when deficiencies are observed with licensing standards. ${ }^{114}$ Depending on a violation's pattern and severity, Oregon's AL/RC settings may have to pay a financial penalty, ranging from less than $\$ 1,000$ to over $\$ 5,000 .^{198}$

In Oregon, state licensing agents conduct inspections at least once every 24 months in AL/RC and memory care-endorsed (MC) settings. ${ }^{122,123}$ Surveyors inspect facility records, including each resident's medication administration record (MAR), which documents the orders (e.g., dose, route, timing), whether the medication is scheduled or pro re nata (PRN; as needed), and any pertinent side effects or interactions. Staff may administer medications, including psychotropic classes, on a PRN basis to treat acute symptoms or supplement scheduled medications. ${ }^{122} \mathrm{AL} / \mathrm{RC}$ staff must describe the parameters for PRN use, individualized to residents. For example, the MAR for a resident 
with a PRN psychotropic medication order for "anxiety" or "aggression" must include a specific description of how that resident exhibits both "anxiety" and "aggression" and non-pharmacologic efforts that staff should attempt before administering medication. ${ }^{78,141,199}$

Little is known about regulatory deficiencies in AL/RC settings, and even less about medication-related deficiencies. Within the AL/RC context, direct care staff roles present an additional layer of complexity to understanding organizational compliance with medication administration regulations..$^{200-202}$ These staff are first line responders to residents' behavioral expressions but cannot formally assess or evaluate. ${ }^{12,141,142,203}$ This study employs applied thematic analysis to examine patterns of organizational noncompliance with psychotropic medication AL/RC rules in Oregon.

\subsection{Methods}

\subsubsection{Data Sources}

This study uses publicly available administrative documents from Oregon's LongTerm Care Licensing website. ${ }^{204}$ This website hosts the last five years of routine inspection and complaint investigation reports, which contain deficiency citations. We downloaded psychotropic medication deficiency citations (C330 tags) issued from 20152019 into Microsoft Excel $(\mathrm{n}=170)$ and then imported into ATLAS.ti, a qualitative analysis software. ${ }^{205}$ Deficiency citations include three types of violations: abuse, licensing, and failure to self-report. Licensing violations represent "failures to substantially comply with licensing rules" as determined by the survey team and include narratives that describe the nature of the violation. ${ }^{198}$ Along with the deficiency citation, 
surveyors and AL/RC staff co-develop "plans of correction" describing the actions staff and management plan to take to both reconcile the deficiencies and prevent them from reoccurring. Plans of correction address the following questions: "what action will be taken to correct the rule violation?", "how will the system be corrected so this violation will not happen?", "how often will the area needing correction be evaluated? and "who will be responsible to see that the correction area is completed/monitored?"

Setting characteristics include licensed capacity (number of beds), license type (AL/RC and MC), whether the setting accepts Medicaid clients, geographic designations (urban/rural), and ownership status (profit/nonprofit). Other sources for setting characteristics include publicly available rosters of the currently licensed AL/RC/MC settings in Oregon (DHS Rosters), the Oregon Office of Rural Health geographic designations by zip code, ${ }^{206}$ and Oregon Secretary of State’s Business Registry. ${ }^{207}$

\subsubsection{Applied Thematic Analysis}

Applied thematic analysis results in identification and description of implicit and explicit ideas within a textual data set. ${ }^{208,209} \mathrm{We}$ aim to identify descriptive and latent themes associated with AL/RC settings' organizational noncompliance with psychotropic medication regulatory requirements. In this study, we used both deductive and inductive coding approaches, reflective memos to describe emergent themes, and discussion among authors to determine interrater reliability. Thematic analysis lends itself to both deductive and inductive coding procedures to further contextualize and reflexively identify overarching patterns. ${ }^{210,211}$ Oregon Administrative Rules found in Chapter 411 Division 54 Section 55-6 were used to deductively define the initial set of codes. ${ }^{122}$ These codes 
parallel the reasons a setting could be cited for noncompliance: "lacking documentation of attempted Nonpharmacological interventions," "lacking evaluation and service planning for Nonpharmacological interventions prior to requesting psychotropic medications," "lacking documentation of resident-specific parameters for use of psychotropic medication," and "not consulting a health professional prior to requesting psychotropic medication." Reading through the deficiency citations revealed patterns related to the deficiencies beyond explicit regulatory noncompliance, leading to the formation of additional codes: the types of psychotropic medications used, whether multiple psychotropic medications were ordered for the same resident, the reasons medications were prescribed, staff roles implicated, and immediate and long-term strategies to reconcile the deficiency and prevent it from happening in the future (e.g., updating resident-specific parameters and nonpharmacologic interventions, comprehensive administration record audits, in-service training for care staff and management).

The entire citation was considered the unit of analysis, however the authors noted when multiple residents were discussed within the citation. For example, a citation could only reflect deficiencies found within one resident's medication records, among multiple residents' records, or general staff practices (not specific to any resident). If a surveyor indicated they reviewed three residents' records and found that two of those records lacked documentation of nonpharmacologic practices, those deficiencies would be coded separately for each resident. The authors individually coded the same ten deficiency citations and discussed coding decisions and additional considerations for interpreting 
surveyor comments. The first author maintained analytic memos to describe emergent themes while coding and discussed findings with the second author during biweekly meetings over a four-month period.

\subsection{Findings}

\subsubsection{Setting Characteristics}

Between 2015-2019 state surveyors issued 170 psychotropic-medication (C330) citations to $152 \mathrm{AL} / \mathrm{RC}$ settings ( $30 \%$ of all settings). Most settings that received C330 citations had an MC endorsement (54\%), followed by 29\% AL only, and 17\% RC only. Nearly all cited settings operated as for profit (96\%), and over half were in urban counties (55\%), compared to $38 \%$ in rural counties and $5 \%$ in frontier counties. Eighty percent of the cited settings accepted Medicaid payment. Capacity ranged from seven beds to 153 beds, and two-thirds of settings had a capacity of 54 beds or less.

Among AL/RC settings who received a C330 deficiency citation between 20152019, surveyors examined at least 292 residents' records with PRN psychotropic medications. Surveyors found deficiencies in $251(86 \%)$ of these records. The primary reasons for deficiency citations included lack of documentation of attempted nonpharmacological interventions and resident-specific parameters indicating use of PRN psychotropic medications, and lack of consultation with healthcare providers prior to requesting PRN psychotropic medications. Emergent themes and related subthemes are detailed below.

\subsubsection{Theme 1: Resident records lacked detailed documentation of administration instructions and attempted nonpharmacologic interventions}


Documentation errors comprised the vast majority of psychotropic medication deficiencies. Across the 170 citations, lacking documentation of attempted nonpharmacological interventions and resident-specific parameters were coded 188 and 130 times, respectively. Residents' medication administration records cited for lacking documentation of nonpharmacological interventions encompassed several scenarios. The most frequently recorded scenario was that residents' records lacked evidence that nonpharmacologic interventions were developed or attempted and failed to specify behavioral descriptions indicating the need for a PRN psychotropic medication. For example,

"There was no documentation non-drug interventions had been attempted with ineffective results prior to administering the PRN psychotropic medication. Progress notes for the dates given showed inconsistent references to failed interventions, and no description of the behavior that required the medication."

Sometimes nonpharmacologic interventions had been developed for staff to attempt, but not documented on the administration record:

"Staff were to try at least three non-drug interventions prior to giving Ativan, which were listed beneath the Ativan order. During 2/2015, Resident 6 was given PRN Ativan on 18 occasions for "yelling" or "yelling and agitation." On 15 occasions, there were no documented nondrug interventions attempted prior to giving the Ativan. Results were not documented on most of the occasions. During 3/2015, Resident 6 was given PRN Ativan on 20 occasions for "yelling" or "yelling \& agitation." No non-drug interventions were indicated on any of the occasions and results were not indicated on most of the occasions."

Subtheme 1a. Response to residents' behavioral expressions are not customized

to the individual. Settings that documented available nonpharmacologic interventions for 
staff to attempt did not ensure these interventions were individualized to any particular resident:

"There was no documented evidence the facility had written resident specific non-pharmacological interventions to be tried prior to administration. In an interview, 3/27/18 at 12:43 pm, Staff 7 [caregiver/medication aide] confirmed the facility used the form, "Behavior Interventions Before Using", for all residents who were prescribed PRN behavior medications. The form had a list of eight nondrug interventions to try prior to administration, however was not resident specific."

Often PRN psychotropic medication parameters included a one-word rationale (eg, anxiety or agitation). Surveyors noted that residents' records were lacking descriptions of how residents expressed clinical indications such as "anxiety," agitation," "restlessness," or "paranoia." For example, one citation described a resident's PRN psychotropic medication orders:

"Resident had signed physician orders for the following PRN psychotropic medications to treat behaviors: Haloperidol $0.5 \mathrm{ml}$ every 4 hours as needed for agitation or nausea. Lorazepam $0.5 \mathrm{mg}$ tablet every 4 hours as needed for anxiety or breathing problems associated with anxiety. Resident 1's MARs, reviewed between 4/1/18 and 6/10/18, revealed the following deficiencies: The MARs failed to include resident-specific parameters which described how Resident 1 exhibited "agitation" and "anxiety." As a result, staff were unclear as to when to administer each medication."

\subsubsection{Theme 2: Unclear parameters place direct care workers in a role paradox.}

In some circumstances, the prescriber's instructions for medication administration left room for interpretation, placing unlicensed direct care staff in a position to overstep their defined roles. Staff who administer PRN medications were left to decide how to do so if MARs did not describe residents' behaviors or medication indications. For example, 
one resident had a medication order for a PRN antipsychotic medication with multiple dosages,

"The current MAR indicated PRN Haloperidol Lactate Concentrate 2 $\mathrm{mg} / \mathrm{ml}$ for delirium or nausea - give 0.25 every 4 hours, or give .5 every 4 hours. Non-licensed staff were left to decide which dose of Haloperidol to administer and what behavioral symptoms the resident might exhibit indicating a need for the medication."

Another resident had multiple PRN orders for psychotropic medications for "anxiety," "sleep," and "agitation." However, the lack of specific parameters left room for unlicensed care staff to interpret when to give which medication,

"The current MAR included lorazepam (anti-anxiety), one to two tablets every four hours as needed for "anxiety or sleep" and haloperidol (antipsychotic) $2 \mathrm{mg} / \mathrm{ml}$ concentrate $0.5 \mathrm{ml}$ by mouth or under tongue every 6 hours as needed for "agitation." Non-licensed staff were left to decide how many tablets of lorazepam to administer and what behavioral symptoms the resident might exhibit indicating a need for the medication. Staff were also left to decide how Resident 3 might exhibit agitation, indicating a need for PRN haloperidol."

Sometimes residents receive PRN psychotropic medications for reasons not

prescribed or indicated as a parameter. For example, one resident had a PRN

benzodiazepine order for "anxiety or shortness of breath." The surveyor noted the

following deficiency,

"Resident was administered Lorazepam for "agitation and aggression".

There was no documented evidence that Resident 1 was displaying anxiety or shortness of breath when the medication was administered. On $11 / 18 / 18$, she was administered Lorazepam for "agitation and inappropriate behaviors" and there was no documentation that nonpharmacological interventions were tried and ineffective before the medication was given."

3.4.4 Theme 3: Disconnect regarding when to seek qualified expertise prior to requesting psychotropic medication from residents' primary care providers 
$\mathrm{AL} / \mathrm{RC}$ operators and staff must consult with a licensed healthcare professional prior to requesting psychotropic medications to determine appropriateness and rule out other potential causes of concern. Surveyors issued deficiency citations when unlicensed AL/RC staff requested psychotropic medications without first consulting with the facility RN. For example,

"Resident's progress notes and interviews with staff revealed unlicensed staff contacted the physician on multiple occasions to request routine and PRN psychotropic medications to treat behavioral symptoms. There was no documented evidence Staff 3 (RN Consultant) was consulted or directed staff to contact the physician."

These citations included evidence of direct care staff or administrators contacting physicians' offices directly through fax and requesting medications by name or asking for medications to treat specific behaviors:

"A fax was sent to the resident's physician from caregiving staff. The fax indicated 'Can we have an order for Lorazepam PRN for [resident name]. [They have] an order for Lorazepam $0.5 \mathrm{mg}-1 / 2 \mathrm{tab}$ before showers. Res seems very anxious, aggitated [sic]. Screaming and yelling. Thank you.' The physician responded with the order as requested. There was no documented RN assessment of the need for an increase in the resident's Lorazepam order."

\section{Subtheme 2a. Working with third party hospice services and staff introduces}

complexity and confusion about responsibility. Oregon rules require clinical

consultation prior to requesting psychotropic medications except for hospice recipients, though other PRN psychotropic requirements remain. For example, one setting received a citation for lacking resident-specific parameters and evidence of nonpharmacologic interventions for a resident receiving hospice services,

"Resident 2 had orders for Lorazepam PRN for anxiety or insomnia and Haldol PRN for agitation and/or hallucinations. A description of the 
behaviors that warranted the medications was lacking and there was no documented evidence other factors had been ruled out for the resident's behaviors including pain, and lack of bowel management. Non-medication approaches to attempt were not identified and per the 3/1-3/31 and 4/14/30/19 MAR noted "not applicable-Hospice." There was no order from Hospice to not attempt non-medication approaches."

\section{Subtheme 2b. Interdisciplinary collaboration can mitigate role confusion. Often}

registered nurses oversee medication management. Some organizations may contract with a consultant pharmacist to assist with medication review and recommendations. One setting described collaborating with their institutional pharmacy to ensure clarity and presence of appropriate clinical indications and PRN psychotropic medication orders,

"The Executive Director and RN will meet with the institutional pharmacy to ensure that the prn parameters created by the nurse will be carried over onto the electronic medication administration records when the pharmacy sends out refill cards. There are times that those parameters are not carried over when the pharmacy updates the medical records to match the new barcode on a bubble pack card within the electronic system. The RN will inservice [sic] medication aids to ensure they understand the importance of documenting their attempts at non-pharmacological interventions prior to administration of PRN psychotropics."

\subsubsection{Theme 4: Going through the motions or driving change: Who are plans of correction for?}

To prevent future deficiencies, setting staff must propose a system change. For example, some settings provide care staff with a visual cue in the form of an order note, "All residents with orders for PRN psychotropic medications will have non-pharmacological interventions added to their MARS as an 'attempt first order" or require action within the electronic administration record, "Intervention check boxes will be added to the MAR and interventions must be entered before 
staff can document medication administration." Plans of correction varied in level of detail and specificity across settings.

Another common proposed plan of correction includes conducting in-service training on medication management, administration, documentation, and regulations for care staff, medication technicians, and management staff (e.g., executive director, administrator, registered nurses). Surveyors described training as both in house (conducted by the affiliated registered nurse) or external educators (e.g., consultant pharmacist). Plans of correction did not detail the content or frequency of these training sessions. Some settings described interdisciplinary plans of correction that included multiple types of staff within the setting, residents' families, pharmacists, and physicians.

One setting planned to implement daily PRN medication order checks, tracking of PRN administrations, and updating training for new hires:

"Resident Coordinators will check daily through all given PRN medications and check documentation of their staff to ensure all prior nonpharmacological interventions had been attempted and documented before administering the medication. Resident Coordinators will report monthly, to nursing, if any PRN medications are being used 3 or more times in a month. This will ensure accurate tracking by the nurses or nurse practitioners of the frequency of PRN's given. During the end of the month medication cycle fill, the Resident Coordinators will double check each medication order to ensure that reasons for use are added and parameters/steps are clear. While training new staff all supervisors will teach required residential care rules around using PRN psychotropic medications and proper non-pharmacological interventions and documentation for each resident in their home."

\subsection{Discussion}

Although psychotropic medication administration rates have been examined, to our knowledge, this is the first qualitative analysis investigating the scope of 
psychotropic medication deficiency citations in AL/RC settings. This study provides important context for understanding state oversight and enforcement of this important quality of care topic. Over half of settings that received a psychotropic medication citation had an MC-endorsement. This is expected given higher use of psychotropic medications among individuals with an ADRD diagnosis. ${ }^{17,32,108,212}$ In Oregon, an estimated $44 \%$ of MC residents received an antipsychotic medication in the prior 90-day period, compared to $17 \%$ in $\mathrm{AL}$ and $21 \%$ in RC settings. ${ }^{121}$

Most citations addressed a lack of documentation of attempted nonpharmacologic practices or resident-specific descriptions of behaviors that warrant administration of a PRN psychotropic medication. This echoes findings of other studies suggesting the majority of deficiency citations issued in long-term care settings do not present imminent danger to residents. ${ }^{24,188,189,191,192}$ Lack of documentation might not mean that staff did not attempt a nonpharmacologic intervention prior to administering a PRN psychotropic medication. However, AL/RC operators' documented reasons for attempted nonpharmacologic interventions provides one level of evidence to state surveyors that required practices actually occurred. Documentation also provides an information source for care staff to make decisions about resident care. ${ }^{146,213}$

An in-depth ethnographic study of technology use in AL/RC reported that staff are "overwhelmed by paperwork [...] they feel draws them away from focusing on working with clients." ${ }^{214}$ Perhaps most importantly, long-term care staff are underpaid and under-resourced for the amount of care they are expected to provide to residents, forcing staff to prioritize. ${ }^{215,216}$ It is possible that other tasks such as documentation and 
charting, though important, become a lower priority in an effort to meet the needs of residents and perform caregiving tasks. Future studies could investigate whether and to what extent regulations unintentionally incentivize documenting tasks at the expense of person-centered care, and if any existing regulatory approaches strike a balance between the two in the AL/RC context.

In addition to documentation errors, the way PRN medications are ordered place direct care staff in a complicated position regarding the scope of their role regarding medication administration. Regulations do not permit paraprofessional care staff to evaluate or make decisions regarding treatments for residents and must deliver treatment and medications as ordered by prescribers. The citations included in this study did not capture the scenario of resident requests as they relate to PRN medications. Residents may request certain as-needed medications from caregivers, who then decide whether to facilitate the administration or not. ${ }^{141,172,203}$ In citations where the state agent listed relevant medication orders for an individual resident, there was frequent co-prescription of PRN antipsychotic and benzodiazepine medications. In some cases, a PRN benzodiazepine was ordered for a resident's “agitation" and a PRN antipsychotic order was in place for "severe agitation." Though direct care staff cannot formally assess or evaluate residents, multiple medications orders and vague parameters put direct care staff in a position where they can and do make these decisions in practice.

Unlicensed care staff may have a role in administering medications to residents. ${ }^{140,193,217}$ Oregon's Board of Nursing rules permit registered nurses to delegate nursing tasks to unlicensed care staff. ${ }^{147,193,218}$ Presence of nurse delegation policies has 
been associated with a larger share of certified (as opposed to paraprofessional) staff handling medication administrations. ${ }^{219}$ Nonspecific descriptions of behaviors assigned to residents, combined with polypharmacy, increase the risk of medication administration errors, most commonly consisting of documentation inconsistencies. ${ }^{143,194}$ Additionally, unless an AL/RC setting is working directly with a consultant pharmacist or nurse, pharmacy technician staff typically process prescription refills and communicate with physician offices, presenting a potential barrier to oversight. ${ }^{220}$ Explicit study of prescribing and deprescribing practices, assessment for inappropriate medications, staff interpretation of prescriber parameters, and communication strategies among AL/RC staff and prescribers are needed to more comprehensively understand how these citations are associated with care delivery in these settings. ${ }^{221}$

Licensing regulations define psychotropic and PRN medication use in Oregon AL/RC. In some states, like Alabama, AL/RC settings are not allowed to use psychotropic medication to respond to residents' behavioral symptoms under any circumstances. ${ }^{222}$ Other states, such as Idaho, provide explicit guidelines for the circumstances, conditions, and staff training related to psychotropic medication use. ${ }^{223}$ Disconnection between prescriber instructions, processes of care, and regulatory expectations and enforcement have consequences. Studies that have examined the relationship between regulatory oversight and use of antipsychotic medications suggest public reporting and stringency do influence the rate these medications are used. ${ }^{224,225}$ Additionally, previous work has shown how the number and severity of deficiency citations can indicate "quality," though these reported relationships are nuanced and 
complex. ${ }^{184,188,189,192,226,227}$ Identifying AL/RC specific mechanisms for medication prescription and administration is necessary to improve processes of care..$^{27,28}$

\subsubsection{Limitations \& Future Directions}

This study has several limitations. First, the level of detail documented in state surveyor inspections facilitates or inhibits our ability to evaluate the qualitative context of deficiency citations, beyond presence or absence. Future research could incorporate interviews with surveyors, facility staff, and residents to contextualize and improve understanding of quantitative and qualitative findings related to deficiency citations and conceptualizations of quality and safety in long-term care. Second, this study examined $\mathrm{AL} / \mathrm{RC}$ regulatory requirements in a single state and may not apply to the regulatory and practice environments of psychotropic medication use among $\mathrm{AL} / \mathrm{RC}$ residents in other states. Psychotropic medication use and regulatory requirements may differ based on the licensing and classification within states. Variation in regulations and resident populations among $\mathrm{AL} / \mathrm{RC}$ settings across the U.S., merit comparison of psychotropic medication use in $\mathrm{AL} / \mathrm{RC}$ settings between and within states to inform relevant policy action. Third, this study focused on psychotropic medication deficiency citations and cannot speak to the greater context of organizational practices or compliance with licensing regulations, which may relate to psychotropic medication use. Relatedly, the extent to which $\mathrm{AL} / \mathrm{RC}$ settings implement the proposed plans of correction or whether these plans are effective at reducing deficiencies are not reflected in these data. Fourth, examining psychotropic medication deficiency citations does not provide a comprehensive perspective of nursing and direct care staff administration decision 
making. Understanding the context of these practices requires review of resident MARs to assess the prevalence and frequency of psychotropic medication prescription and administration within AL/RC settings.

\subsubsection{Conclusions \& Implications}

Documentation errors comprised the majority of psychotropic medication deficiency citations issued to Oregon AL/RC settings from 2015-2019. By examining deficiency citations, policy makers, operators, and staff can identify (in)congruence between regulatory expectation and practical reality. Policymakers can consider how regulations may unintentionally incentivize task-oriented versus person-centered care practices and incorporate $\mathrm{AL} / \mathrm{RC}$ staff perspectives in policy development. Citations do not fully capture the upstream circumstances that may lead to organizational noncompliance including physician prescribing practices, staff resources and support, and industry influences (eg, revenue, pharmaceutical culture, operationalization of behavioral expressions associated with ADRD/cognitive impairment). 
Chapter 4: Antipsychotic Medication Use in Assisted Living/Residential Care: Do Organizational Characteristics Matter?

\subsection{Abstract}

Objective: To investigate how the assisted living/residential care (AL/RC) and memory care (MC) contexts are associated with the prevalence of antipsychotic medication use (APU).

Data sources: Primary data were collected from a statewide representative sample of AL/RC settings through the Oregon Community-Based Care study from 2017-2019 and combined with publicly available administrative data.

Study Design: Framed by Donabedian's model of care quality, we examine associations among 90-day prevalence of APU, organizational, care process, and AL/RC resident population characteristics using random intercepts regression models.

Data Collection: Every licensed AL/RC setting in Oregon receives an annual mailed survey to provide aggregate resident demographics, health acuity, health service use, payment type and organizational policies. Organizational measures (e.g., profit status, license type, geographic designation) were collected from state websites.

Principal Findings: The average 90-day prevalence of APU among all Oregon AL/RC settings is $30.7 \%$, though rates differ by MC endorsement (23.9\% in AL/RC and 42.7\% in MC). Compared to care processes and resident population characteristics, organizational characteristics were associated with larger magnitudes of difference in rates of APU. Nonprofit settings were associated with lower rates of APU in both AL/RC $(\beta=-4.4$ (percentage points), [95\% CI: $-8.4,-0.4])$ and $\mathrm{MC}(\beta=-12.4$, [95\% CI: $-21.2,-$ 3.6]. Compared to low-Medicaid settings, settings with very high proportions of 
Medicaid residents were associated with higher prevalence of APU, +8.9 in AL/RC (95\% CI: $1.7,16.1)$ and +11.0 percentage points in MC $(95 \%$ CI: $2.3,19.8)$.

Conclusions: APU prevalence in MC settings is considerably higher than other longterm care options. Additional study is needed to contextualize the relationships between $\mathrm{AL} / \mathrm{RC}$ population-level practices and characteristics and the prevalence of APU to inform policy and practice development related to this measure as a quality indicator. 


\subsection{Introduction}

Assisted living and residential care (AL/RC) settings are increasingly important providers of long-term services and supports for older adults in the United States (U.S.), outpacing nursing home (NH) availability. ${ }^{53,54}$ Approximately 29,000 AL/RC settings are home to $\sim 812,000$ residents. ${ }^{1}$ One quarter of AL/RC settings report at least $75 \%$ of their resident populations have an Alzheimer's disease or related dementia (ADRD) diagnosis. ${ }^{228}$ The estimated prevalence of persons living with ADRD ranges from 42 to $72 \%$ in these settings. ${ }^{1,2}$ Residents with cognitive support needs commonly express behaviors, which can manifest as a means of communication, response to environmental stimuli, or reaction to pain or discomfort. ${ }^{3,4,6,60,74}$ Behavioral expressions associated with ADRD include agitation, wandering, sleep disturbances, hallucinations, and delusions..$^{57,8,59}$ These behaviors can result in considerable distress to the resident and their family, and other residents and care staff. ${ }^{11,12,67-69,229}$

Residents' quality of care and life can depend on appropriate understanding of and responses to the underlying causes of behavioral expressions through a balance of psychosocial and medication-related interventions. ${ }^{4,13,15,72,230}$ Common pharmaceutical approaches to manage behaviors expressed by people living with ADRD include psychotropic medications (ie, benzodiazepines, antipsychotics, sedatives, anxiolytics, and antidepressants), though risks associated with these medications must be balanced with potential benefits. ${ }^{16-18,77,81,131,231}$ Clinical practice guidelines recommend antipsychotic medications, in particular, only for severe psychotic symptoms unresponsive to nonpharmacologic treatment. ${ }^{77,232,233}$ Specifically for people living with ADRD, 
antipsychotic medication use (APU) has been associated with adverse outcomes including falls, sedation, cognitive decline, and mortality. ${ }^{19-22,108,212,234,235}$

Much attention has been paid to reducing inappropriate APU in NH, though people living with ADRD reside in various care settings that warrant similar intervention. ${ }^{27,28,181}$ One recent study found higher prevalence of potentially inappropriate APU in AL/RC settings compared to NH. ${ }^{23}$ Both the Centers for Medicare and Medicaid Services (CMS) and industry organizations identify APU as a top priority in the context of long-term care quality improvement, though explicit initiatives to reduce APU in older adults with ADRD have largely focused on NH settings..$^{25,26,102}$

Many characteristics should be considered in the context of quality improvement. Researchers studying quality of life and quality of care in long term supports and services conceptualize organizational characteristics such as ownership, chain membership, size, occupancy, and Medicaid census as indicators of a settings' ability to meet resident needs. ${ }^{236,237}$ Recent reviews have identified medication management as a relevant quality domain pertaining to $\mathrm{AL} / \mathrm{RC}$ settings, encompassing APU. ${ }^{238,239}$ The prevalence of AL/RC residents living with ADRD and ongoing efforts to improve quality of care warrant the investigation of APU as a quality indicator in relation to other characteristics deemed relevant to quality measurement.

$\mathrm{AL} / \mathrm{RC}$ settings offer different levels of care to meet the individual needs of residents who live with complex health conditions, medication regimens, and often receive assistance with activities of daily living. Almost two-thirds of residents receive bathing assistance overall, ranging from $48 \%$ to $86 \%$ depending on the prevalence of 
dementia. ${ }^{1,228}$ Dementia care, or memory care (MC), units are licensed and marketed to provide care for people living with ADRD. Each state regulates and oversees AL/RC settings, determining licensing, resident admission/discharge, staffing levels, and dementia care practices criteria, resulting in within and between state variation. ${ }^{111,112,197}$ Variation in populations served and practices enacted by different types of licensed AL/RC settings warrants intrastate investigation. In Oregon, $\mathrm{AL} / \mathrm{RC}$ settings operate under the same regulations, but differ in the physical environment: AL settings must have private bathrooms and kitchenettes, where RC settings may have shared bathrooms and kitchens. ${ }^{122}$ Both AL/RC settings can have MC endorsements, catering to residents diagnosed with ADRD and included additional dementia-specific training and staffing requirements. ${ }^{123}$

\subsubsection{Conceptual Model}

This study employs Donabedian's model of care quality. While this framework was developed to assess quality of medical care delivery, it has been expanded upon and applied to other health services and long-term care settings. ${ }^{239,240,240-244}$ Foundationally, Donabedian's model categorizes sources of information related to quality in healthcare and health services in three areas: structures, processes, and outcomes. Structures comprise the organizational characteristics of a health organization and are referred to as "organizational" in this study. Processes encompass how providers and receivers of health services interact, and outcomes are broadly considered as the individual health statuses of those receiving health services. 
Figure 1. Application of Donabedian's model to antipsychotic medication use in assisted living/residential care settings.

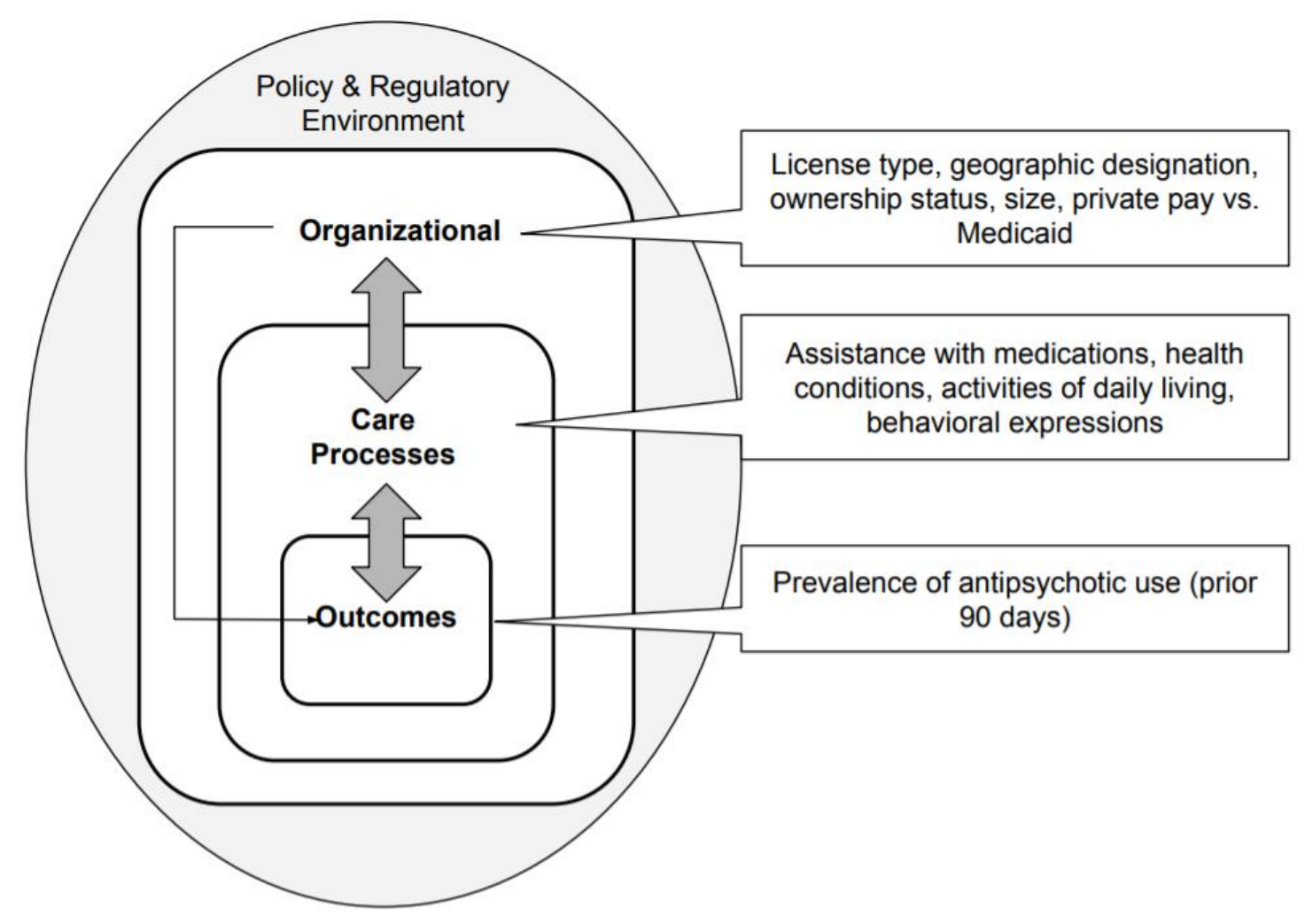

We apply Donabedian's model to examine the relationships among proxy measures for organizational characteristics and care processes associated with the prevalence of APU in AL/RC populations (Figure 1). Care processes of interest include medication administration, hospice service use, and assistance with behaviors. Regulatory oversight of licensed care settings can facilitate or inhibit organizations' abilities to meet residents' needs. ${ }^{114,245}$ While processes and practices within AL/RC settings are theoretically driven by residents' needs, the policy and regulatory environment shapes the underlying structure of $\mathrm{AL} / \mathrm{RC}$ settings via licensing and oversight. We explore the extent 
to which the residential/organizational context affects how organizational characteristics and care processes are associated with the prevalence of APU.

\subsection{Methods}

\subsubsection{Study Design and Sample}

We conduct a secondary data analysis of the annual Oregon Community-Based Care (CBC) Study of AL/RC settings operating in Oregon, where the AL/RC setting is the unit of analysis. ${ }^{121,246,247}$ The study team mails paper surveys to all actively licensed AL/RC settings in Oregon. Those who responded to the 2017-2019 waves of the CBC study were included in this analysis. Not every setting participated in all three study waves, resulting in an unbalanced panel (195 of settings participated in three waves, 171 in two waves, and 118 in one wave). All variables included in the analysis had less than 5\% missing information. The final analytic sample includes 932 observations after listwise deletion (11\% reduction) from 463 (4\% reduction) settings across 2017-2019 (137 of settings participated in all three waves, 195 in two waves, and 131 in one wave).

\subsubsection{Data Sources}

As explained above, this study utilizes three waves (2017-2019) of the Oregon CBC study. ${ }^{121,246,247}$ which provides aggregate AL/RC setting-level characteristics. We used publicly available DHS/APD rosters of currently licensed settings to source organizational characteristics (e.g., licensed capacity, license type, Medicaid contract). ${ }^{204}$ We then matched settings' zip codes to service area maps that designate urban and rural geography. ${ }^{206}$ Finally, we used the most recent business filings on the Oregon Secretary of State's website to identify each settings' registered owners' profit status. ${ }^{207}$ 


\subsubsection{Measures}

Outcome measure. AL/RC setting prevalence of APU, defined as the proportion of residents within each setting who received antipsychotic medication in the past 90 days.

Organizational characteristics. Setting size, calculated using the highest number of residents a settings are licensed to accommodate, was categorized as: small (7-24 beds), medium ( $25-49$ beds), large (50-74 beds), and very large (75 or more beds). ${ }^{248}$ License type identifies the regulatory certification of the setting: $\mathrm{AL} / \mathrm{RC}$ or $\mathrm{MC}$. Geographic designation and profit status are dichotomous measures: urban or rural/frontier counties and for profit/nonprofit. Medicaid census categorically measures the percent of residents using Medicaid to pay for services, divided into four categories using thresholds based on quantile distributions: low $(0 \%$ - 15\%), moderate $(15.1 \%-$ $43.1 \%)$, high $(43.2 \%-67.7 \%)$, and very high $(67.8 \%-100 \%)$.

Process characteristics. We use two measures as proxies for relevant care processes within an $\mathrm{AL} / \mathrm{RC}$ setting: percentage of residents who receive staff assistance to take oral medications, and a constructed "behavioral needs index." Receiving medication assistance is associated with other functional assistance in addition to caregiving time. ${ }^{249}$ The latter provides a measure of the prevalence of behavioral needs across settings and is based on a count of residents who need regular and ongoing assistance with each of three behavioral expressions: 1) lack of awareness of safety, judgment, and decision making, or ability to orient to surroundings, 2) wandering, and 3) danger to self or others. The behavioral needs index is constructed by adding together the 
percentage of residents needing assistance with the aforementioned behaviors divided by the number of behaviors, resulting in a scale from 0 (no behavioral needs) to 100 (all residents receive assistance with all three behavioral needs).

Covariates. Characteristics of settings' resident populations will be used as control measures within the regression models, based on findings from previous studies. ${ }^{125,132,134,250}$ The average age of residents is reported in years. We also report setting-level prevalence of residents by sex, race/ethnicity, diagnosed with ADRD or serious mental illness, and those who used hospice services within the past 90 days. We collected data on racial/ethnic categorization in this survey based on U.S federal government standards: American Native/Alaska Native, Asian, Black/African American, Hispanic/Latino, Native Hawaiian/Pacific Islander, White, or multiracial. ${ }^{251}$

\subsubsection{Analytic Approach}

We use means, standard deviations, and proportions to present summary statistics. We employ multilevel models in this study, where survey waves are nested within settings. First, we assessed bivariate associations between the dependent variable and organizational, process, and covariate characteristics using unadjusted setting-level random intercepts regression models stratified by license type (AL/RC vs. MC), and then estimate setting-level random intercepts regression models to test associations of organizational characteristics and proxy measures of care processes, adjusting for grouplevel covariates. Random intercepts regression is used to assess differences in the prevalence of APU within and between individual settings over time, accounting for $\mathrm{AL} / \mathrm{RC}$ settings that consistently have higher or lower prevalence of APU compared to 
others in the sample. To account for repeated measures from each setting over time, study waves are clustered within settings to ensure robust standard errors and appropriate estimates. ${ }^{252}$ All analyses were conducted using Stata $15 .^{253}$

\subsection{Results}

\subsubsection{Summary Statistics}

Table 1 describes the organizational, care processes, and resident population characteristics of the responding AL/RC settings for 2017-2019 CBC study waves and by license type. Settings ranged in size from seven beds to 186 beds with a median of 48 beds. Most settings operated for profit and in urban counties. A larger share of responding $\mathrm{MC}$ settings operate for profit and in urban counties compared to AL/RC only settings. The average proportion of residents using Medicaid to primarily pay for services is $42.3 \%$ across all license types. A slightly larger share of MC settings (26.6\%) were categorized as very high Medicaid compared to AL/RC settings (23.6\%). Bolded pvalues beside characteristics indicate significant differences at $\mathrm{p}<.05$ between MC and non-MC cases based on t-tests and Pearson's chi-square tests.

Similar to national estimates, residents in our study are mostly female, nonHispanic White, and over 80 years old. ${ }^{1}$ The average proportion of residents diagnosed with ADRD was $54.6 \%$ (median: $43.0 \%$ ) and the average proportion of residents diagnosed with serious mental illness was $8.1 \%$ (median: $4.0 \%$ ). The average proportion of residents receiving regular and ongoing assistance with taking oral medications were 81.9\% (median: 95\%). The average prevalence of APU in the last 90 days was $30.7 \%$ (median: 22.2\%). MC settings had larger average proportions across all care process 
characteristics. The average proportion of residents receiving hospice care or antipsychotic medications in the last 90 days were also higher in MC settings. AL/RC settings had a larger average proportion of residents with a diagnosis of SMI, though across all settings the average SMI prevalence was below $10 \%$.

Table 1. Proportions, means, and standard deviations of responding assisted living/residential care and memory care setting characteristics, 2017-2019.

\begin{tabular}{|c|c|c|c|c|}
\hline Characteristics & $\begin{array}{l}\mathrm{AL} / \mathrm{RC} \\
(\mathrm{n}=598) \\
\end{array}$ & $\begin{array}{c}\text { Any MC }^{\mathbf{a}} \\
(\mathrm{n}=334)\end{array}$ & $\begin{array}{l}\text { Overall } \\
(\mathbf{n}=932)\end{array}$ & $\begin{array}{c}\text { p- } \\
\text { value }\end{array}$ \\
\hline \multicolumn{5}{|l|}{ Organizational (\%) } \\
\hline Size: Small; 0-24 beds & 21.7 & 33.5 & 26.0 & \multirow{4}{*}{0.000} \\
\hline Medium; 25-49 beds & 26.1 & 32.6 & 28.4 & \\
\hline Large; $50-74$ beds & 28.1 & 22.5 & 26.1 & \\
\hline Very large; $75+$ beds & 24.1 & 11.4 & 19.5 & \\
\hline Geographic Designation: Urban & 54.8 & 59.6 & 56.6 & \multirow{2}{*}{0.162} \\
\hline Rural/frontier & 45.2 & 40.4 & 43.4 & \\
\hline Ownership: Nonprofit & 14.1 & 8.1 & 11.9 & \multirow{2}{*}{0.007} \\
\hline For profit & 85.9 & 91.9 & 88.1 & \\
\hline Medicaid census: Low (0-15\%) & 27.9 & 19.8 & 25.0 & \multirow{4}{*}{0.040} \\
\hline Moderate $(15.1 \%-43.1 \%)$ & 24.7 & 25.1 & 24.9 & \\
\hline High $(43.2 \%-67.7 \%)$ & 23.7 & 28.4 & 25.4 & \\
\hline Very high $(67.8 \%-100 \%)$ & 23.6 & 26.6 & 24.7 & \\
\hline \multicolumn{5}{|l|}{ Care Processes (mean (SD)) } \\
\hline Taking oral medications & $75.8(30.0)$ & $92.7(22.3)$ & $81.8(28.7)$ & $\mathbf{0 . 0 0 0}$ \\
\hline Behavioral needs index $(0-100)$ & $11.2(14.7)$ & $39.3(17.8)$ & $21.2(20.8)$ & $\mathbf{0 . 0 0 0}$ \\
\hline Lack of awareness of safety & $23.7(29.3)$ & $79.7(29.2)$ & $43.8(39.7)$ & 0.000 \\
\hline Wandering & $5.0(10.2)$ & $29.3(28.2)$ & $13.6(22.0)$ & 0.000 \\
\hline Danger to self/others & $4.8(14.3)$ & $8.9(14.4)$ & $6.3(14.5)$ & 0.000 \\
\hline \multicolumn{5}{|l|}{ Resident Population (mean (SD)) } \\
\hline Average age (years) & 81.8 (7.7) & $83.2(5.3)$ & $82.3(7.0)$ & 0.002 \\
\hline
\end{tabular}




\begin{tabular}{lcccc} 
Female & $68.0(14.4)$ & $71(13.0)$ & $69.0(14.0)$ & $\mathbf{0 . 0 0 5}$ \\
Male & $32.0(14.4)$ & $28.3(13.0)$ & $31.0(14.0)$ & $\mathbf{0 . 0 0 5}$ \\
Hispanic or Latino and/or persons of color & $5.2(11.0)$ & $7.1(13.6)$ & $5.6(12.1)$ & 0.124 \\
$\quad$ Hispanic or Latino, any race & $1.1(2.5)$ & $1.8(3.3)$ & $1.3(2.9)$ & $\mathbf{0 . 0 0 0}$ \\
$\quad$ American Indian/Alaska Native & $1.0(3.9)$ & $1.3(7.9)$ & $1.1(5.7)$ & 0.497 \\
$\quad$ Asian & $0.8(2.2)$ & $1.2(2.3)$ & $1.0(2.3)$ & $\mathbf{0 . 0 0 7}$ \\
$\quad$ Black/African American & $0.8(4.6)$ & $1.0(3.2)$ & $0.9(4.2)$ & 0.460 \\
$\quad$ Native Hawaiian/Pacific Islander & $0.6(5.9)$ & $0.5(5.3)$ & $0.6(5.7)$ & 0.753 \\
$\quad$ Multiracial & $0.8(5.5)$ & $1.2(7.6)$ & $0.9(6.4)$ & 0.386 \\
non-Hispanic/Latino, White & $90.0(21.7)$ & $87.6(24.5)$ & $89.2(22.8)$ & 0.124 \\
Race/ethnicity not disclosed & $4.8(19.2)$ & $5.3(21.7)$ & $5.0(20.1)$ & \\
Alzheimer's or related dementia diagnosis & $33.9(24.0)$ & $91.7(19.6)$ & $54.6(35.7)$ & $\mathbf{0 . 0 0 0}$ \\
(ADRD) & & & & \\
Serious mental illness (SMI) diagnosis & $9.0(17.2)$ & $6.5(10.3)$ & $8.1(15.1)$ & $\mathbf{0 . 0 1 7}$ \\
Receiving hospice services (last 90 days) & $6.7(8.8)$ & $11.0(9.5)$ & $8.2(9.3)$ & $\mathbf{0 . 0 0 0}$ \\
Using Medicaid to primarily pay for & $40.5(31.5)$ & $45.4(29.5)$ & $42.3(30.9)$ & $\mathbf{0 . 0 2 1}$ \\
services & & & & \\
Receiving antipsychotic medication (last & $23.9(23.7)$ & $42.7(23.5)$ & $30.7(25.3)$ & $\mathbf{0 . 0 0 0}$ \\
90 days) & & & & \\
\hline
\end{tabular}

a“"Any memory care" includes settings where MC units are co-located within assisted living/residential care settings as opposed to stand-alone MC settings.

\subsubsection{Random Intercepts Regression of Organizational Characteristics}

Figure 2 shows the coefficient estimates and 95\% confidence intervals for four random intercepts regression models estimating associations among organizational and the prevalence of APU in the prior 90 days, stratified by license type. Bivariate and adjusted model estimates and 95\% CIs are located in more detail in Appendix A, Table 2 and Table 3. 
Figure 2. Results from random intercepts regression analysis of organizational characteristics and prevalence of antipsychotic medication use by setting type, 20172019.

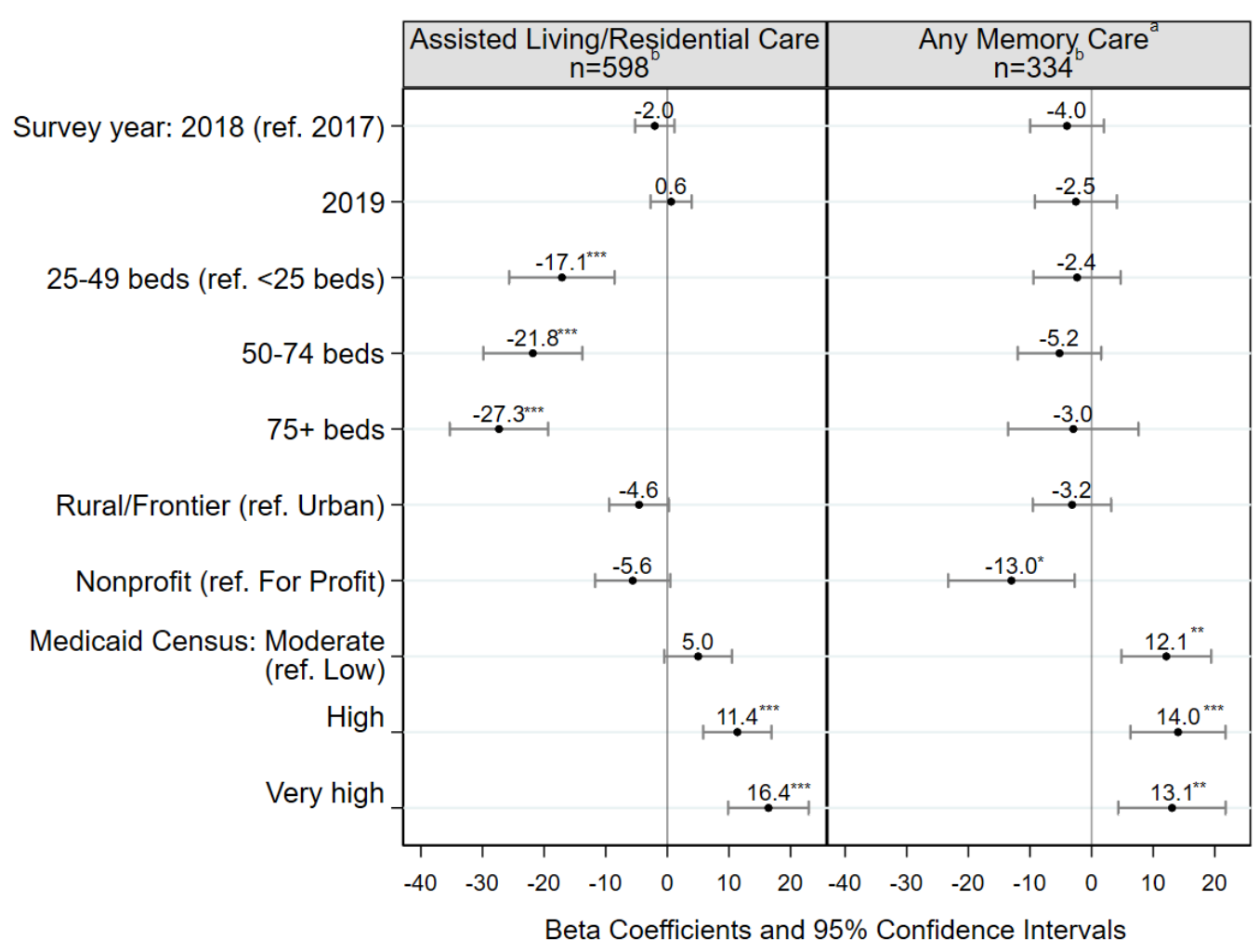

$* \mathrm{p}<0.05, * * \mathrm{p}<0.01, * * * \mathrm{p}<0.001$

a "Any MC" includes memory care units that are co-located within assisted living or residential care settings.

${ }^{\mathrm{b}}$ Over the study period, AL/RC settings comprise 598 observations clustered within 297 settings and MC settings comprise 334 observations clustered within 167 settings.

In bivariate analysis, settings with larger Medicaid censuses were associated with higher prevalence of APU across all setting types (Table 2). Larger AL/RC settings ( $\beta=$ 27.3 (percentage points), [95\% CI: $-35.3,-19.3$ ] and nonprofit MC settings ( $\beta=-13.0$ (percentage points), [95\% CI: -23.3, -2.7]) were associated with lower prevalence of APU. AL/RC setting size and nonprofit MC status remain negatively associated and larger Medicaid census across all settings remain associated with higher prevalence of APU when adjusting for all organizational characteristics (Figure 2, Table 3). Medicaid 
census and setting size appear to show a linear relationship (positive and negative, respectively) with the prevalence of APU among AL/RC settings, but not in MC settings.

\subsubsection{Random Intercepts Regression of Processes of Care and Resident Population}

\section{Characteristics}

Figure 3 shows the coefficient estimates and 95\% confidence intervals for random intercepts regression models estimating associations among measures of care processes and resident population characteristics and the prevalence of APU in the prior 90 days, stratified by license type (Table 2 and Table 3). In bivariate analysis, higher average age and proportions of residents who were female were associated with lower APU prevalence in $\mathrm{AL} / \mathrm{RC}$ settings while proportions of residents diagnosed with $\mathrm{ADRD}$ and SMI, and greater behavioral needs were associated with higher APU prevalence. In MC settings, only the proportion of residents receiving assistance taking oral medications was associated with the prevalence of APU (Table 2). Including all processes of care measures and resident characteristics attenuates the relationship between proportion of female residents and APU, though reflect bivariate associations with other characteristics among AL/RC settings. A one percentage point increase in the proportion of residents with ADRD is associated with a 0.2 percentage point increase in the prevalence of APU, even when adjusting for the proportion of residents diagnosed with SMI (Figure 3, Table 3). In the adjusted model, an increase in the proportion of residents using hospice in the prior 90 days has the same effect on the prevalence of APU in both AL/RC and MC settings. 
Figure 3. Results of random intercepts regression analysis of processes of care and resident population characteristics and prevalence of antipsychotic medication use by setting type, 2017-2019.

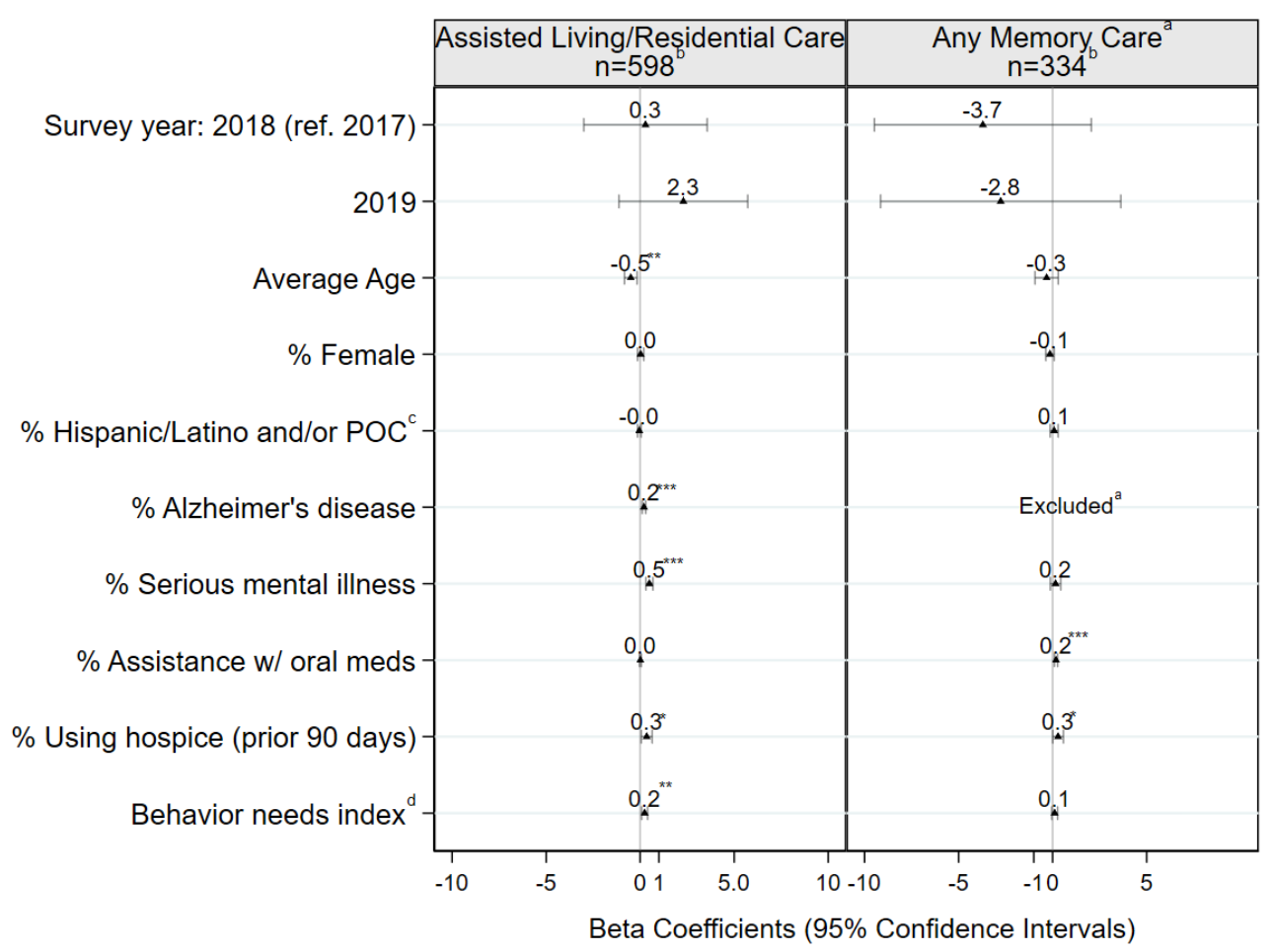

$* \mathrm{p}<0.05, * * \mathrm{p}<0.01, * * * \mathrm{p}<0.001$

a "Any MC" includes memory care units that are co-located within assisted living or residential care settings. Because residents living in memory care must have an Alzheimer's or related dementia diagnosis, the "any MC" model excludes ADRD due to collinearity.

${ }^{\mathrm{b}}$ Over the study period, AL/RC settings comprise 598 observations clustered within 297 settings and MC settings comprise 334 observations clustered within 167 settings.

c "POC"= people of color; Includes residents identified as American Indian/Alaska Native, Asian, Black/African American, Native Hawaiian/Pacific Islander, and multi-racial

${ }^{\mathrm{d}}$ Behavioral needs index $=[(\%$ residents receiving assistance for lack of awareness to safety $+\%$ wandering $+\%$ danger to self/others) / 3] x 100

\subsubsection{Fully Adjusted Random Intercepts Regression Model}

Figure 4 shows coefficient estimates and 95\% CIs for the fully adjusted models (including all organizational, care processes, and resident characteristics stratified by license type (also see Table 4). Overall, the relationships examined in the models only controlling for organizational or care processes and resident population characteristics are replicated in the models controlling for all sets of characteristics. 
Figure 4. Results of random intercepts regression analysis of organizational, processes of care and resident population characteristics and prevalence of antipsychotic medication use by setting type, 2017-2019.

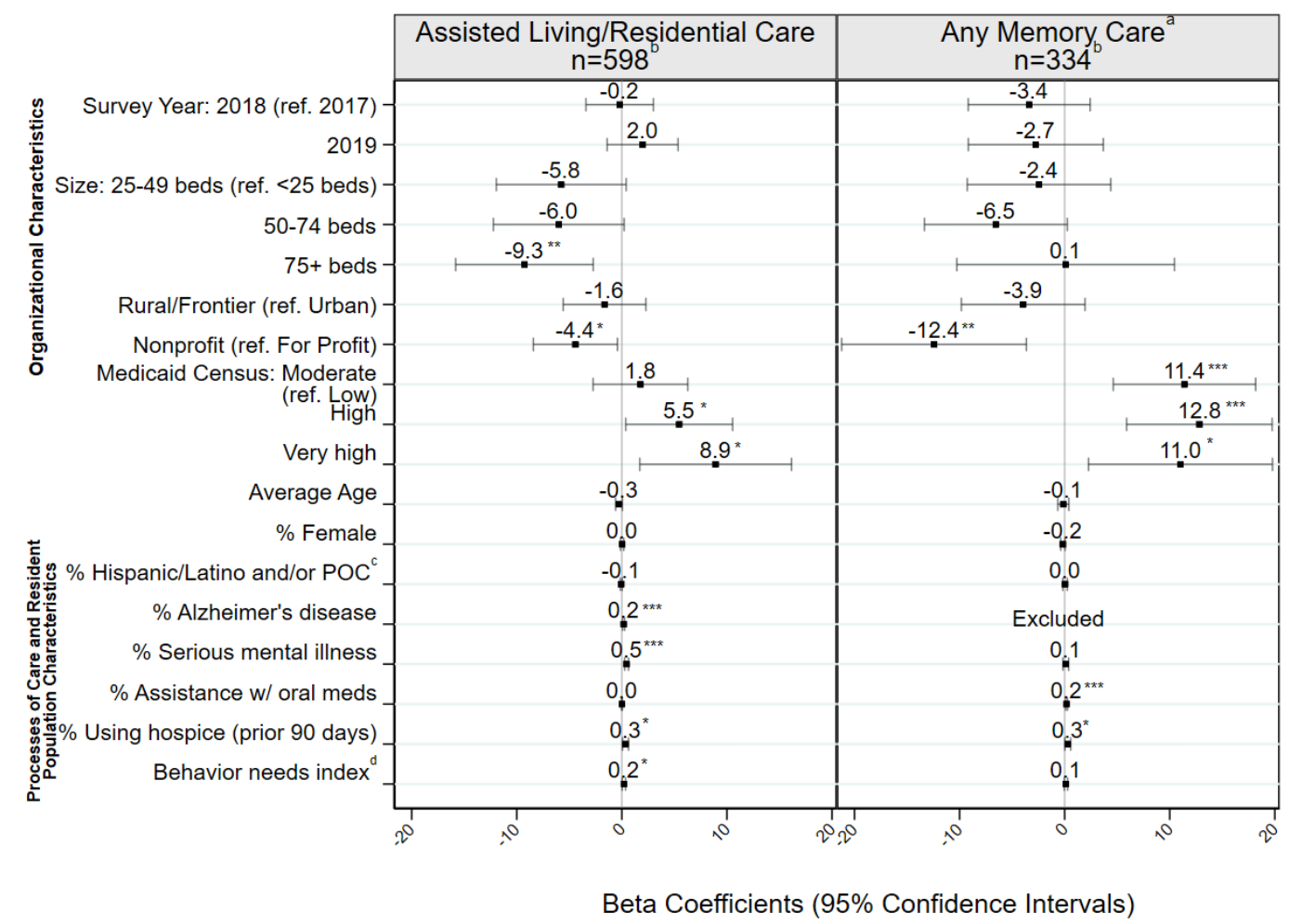

$* \mathrm{p}<0.05, * * \mathrm{p}<0.01, * * * \mathrm{p}<0.001$

a "Any MC" includes memory care units that are co-located within assisted living or residential care settings. Because residents living in memory care must have an Alzheimer's or related dementia diagnosis, the "any MC" model excludes ADRD due to collinearity.

${ }^{\mathrm{b}}$ Over the study period, AL/RC settings comprise 598 observations clustered within 297 settings and MC settings comprise 334 observations clustered within 167 settings.

c "POC"= people of color; Includes residents identified as American Indian/Alaska Native, Asian, Black/African American, Native Hawaiian/Pacific Islander, and multi-racial

${ }^{\mathrm{d}}$ Behavioral needs index $=[(\%$ residents receiving assistance for lack of awareness to safety $+\%$ wandering $+\%$ danger to self $/$ others $)$ /3] x 100

Although the effect size has slightly decreased, measures of size and Medicaid census have similar linear relationships with the prevalence of APU among AL/RC settings compared to bivariate and adjusted models presented Figure 2, Table 2, and Table 3. MC settings do not reflect this linear relationship. Nonprofit status is associated with lower prevalence of antipsychotic use across all settings, though there is a larger effect size for MC settings. Our measure of behavioral needs within MC resident 
populations is not significantly associated with prevalence of APU in any model estimation presented in this study, though it is associated in AL/RC populations.

\subsection{Discussion}

This study utilized three years of facility-level panel data to estimate associations between measures of organizational, processes of care, and resident population characteristics with the average 90-day prevalence of APU among AL/RC and MC settings in Oregon. From 2017-2019, the average 90-day prevalence of antipsychotic use across all setting types was $31 \%$. APU rates differed by license type: $24 \%$ in AL/RC and $43 \%$ in any setting with MC certification. Characteristics associated with differences in average 90-day prevalence of APU include size, profit status, Medicaid census, prevalence of ADRD, serious mental illness, assistance with oral medications, hospice use, and behavioral needs. Despite accounting for characteristics of the resident population, organizational characteristics remained associated with APU rates compared, and license types moderated the patterns of association among these characteristics. This study contributes a representative, statewide, longitudinal analysis of APU in AL/RC settings and resident populations. APU is important because of its prevalence in people living with ADRD, despite associations with adverse outcomes. ${ }^{20,22,23,212,232}$

We found that the APU prevalence in MC settings (43\%) is greater than other long-term care options in Oregon: AL/RC settings (24\%), adult foster homes (35\%), ${ }^{254}$ and NHs (15\%). ${ }^{124} \mathrm{MC}$ residency is conditioned upon a diagnosis of ADRD, and this diagnosis is associated with a higher likelihood of receiving antipsychotic medications..$^{255}$ ADRD prevalence in Oregon differs by setting type: $34 \%$ in AL/RC and $92 \%$ in any MC 
setting, compared to $46 \%$ in adult foster homes ${ }^{254}$ and $18 \%$ in NHs. ${ }^{124}$ Additionally, our prior work showed that individual residents living in MC settings were twice as likely to receive an antipsychotic medication in the prior 90 days compared to AL/RC residents. ${ }^{134}$

We observed a mix of organizational, care processes, and resident population characteristics associated with the average 90-day prevalence of APU. AL/RC resident populations that have greater behavioral needs and prevalence of serious mental illness had higher average prevalence of APU, but not in MC settings. In MC settings, a higher proportion of residents receiving staff assistance with taking oral medications was associated with higher prevalence of APU. As expected, hospice use prevalence was associated with higher prevalence of APU across setting types.

Organizational characteristics are associated with average prevalence of APU regardless of care processes and resident population characteristics. In particular, nonprofit status was consistently associated with lower prevalence of APU and Medicaid census was consistently associated with higher prevalence of APU across setting types. Among AL/RC settings, we observed a linear relationship when comparing the prevalence of APU in the very high Medicaid settings compared to low Medicaid settings (ie, greater percentage of private pay residents). While Medicaid census was also associated with higher APU in MC settings, we did not observe the same linear relationship. At the individual level, receiving an antipsychotic medication in the last 90day period was not associated with a resident's use of Medicaid. ${ }^{134}$ Other studies have reported NH residents primarily using Medicaid were more likely to have inappropriate 
APU than private pay residents ${ }^{256}$ and, similar to our findings, settings with high Medicaid census reported higher APU. ${ }^{257}$

Donabedian's model asserts that a combination of organizational and process characteristics facilitates outcomes used to conceptualize quality. Our finding that settings with higher proportions of residents primarily using Medicaid (based on quartile distributions) have higher prevalence of APU warrants further consideration. Over time, Medicaid has increased as a payment source for home and community based care settings through state waiver programs, though most utilizers are people with intellectual and developmental disabilities (63\%) compared to older adults and people with physical disabilities $(41 \%) .{ }^{258}$ Nationwide, an estimated $48 \%$ of AL/RC communities report Medicaid-certification, with only $17 \%$ of residents primarily using Medicaid to pay for services nationally. ${ }^{1}$ On average, $42 \%$ of Oregon AL/RC residents primarily used Medicaid to pay for services. ${ }^{121}$

Higher Medicaid reimbursement rates have been associated with improved quality measures and resident outcomes among NH residents, including increased continuity of care, staffing levels, and lower hospitalizations, incidence of pressure ulcers, restraint use and APU. ${ }^{102,259-261}$ In Oregon, the 2018 monthly Medicaid reimbursement rate for an AL resident ranged from $\$ 1,234$ (receiving the lowest level of care) to $\$ 2,922$ (receiving the highest level of care) and $\$ 4,063$ for MC residents. ${ }^{262}$ On average, private pay AL and MC residents in Oregon pay an estimated \$3,959 and \$5,620 per month receiving the lowest level of care, respectively. ${ }^{263}$ Additionally, Medicaid does not reimburse for room and board costs in AL/RC. ${ }^{264}$ Settings with a high proportion of Medicaid residents 
operate with less financial resources than settings with high proportions of private pay residents, potentially impacting operations with regard to staff time, and ability to meet residents' needs. ${ }^{265}$ For example, from $\mathrm{NH}$ research indicate a pattern of residents who primarily use Medicaid are clustered in lower quality facilities. ${ }^{266}$

In Oregon, RC settings, which differ from AL in terms of resident population, size, and physical environment, are more likely to have MC endorsements as well as more likely to accept Medicaid residents compared to AL settings, ${ }^{267}$ which may also partly explain the observed association between APU prevalence and a setting's Medicaid census. Over time, the number of settings with MC certification increased from 148 in 2014 to 186 in 2019, (26\% increase over five years). Nationally, an increased supply of AL availability is associated with a decrease in residents who have lower care needs, but qualify for $\mathrm{NH}$, where Medicaid is more widely used. ${ }^{54,119}$ However, Medicaid-eligible $\mathrm{AL} / \mathrm{RC}$ residents tend to have greater needs, higher levels of chronic care conditions, and disability. ${ }^{268}$ Additional time points, accounting for staff levels, composition, and training, in addition to $\mathrm{AL} / \mathrm{RC}$ residents' health- and care-related needs are necessary to further disentangle the relationship between prevalence of APU and proportion of residents using Medicaid. A future study could examine whether increases in MC availability correlate to increases in proportions of Medicaid residents served in AL/RC settings.

In the last decade, there has been an organized effort to reduce off-label APU in $\mathrm{NH}$, incentivized through a direct connection to public-facing quality indicators in these settings, such as 5-star rankings. ${ }^{102,225}$ AHCA/NCAL (2020) has a quality initiative 
encouraging $\mathrm{AL} / \mathrm{RC}$ settings to reduce the rate of off-label APU to $15 \%$, mirroring similar efforts in NH. ${ }^{26}$ Similarly, Oregon's 2017 legislative session passed two bills regarding $\mathrm{AL} / \mathrm{RC}$ quality of care, one to reduce medication errors ${ }^{136}$ and the other reduce adverse side effects and nonstandard long-term use of antipsychotic medications. ${ }^{39}$ Policymakers might consider potential differential impact by using APU prevalence as a quality indicator. Given the heterogeneous structure of $\mathrm{AL} / \mathrm{RC}$ regulations, organizational resource capacity, and care needs among resident populations, a pre-set threshold of APU does not explicitly address appropriate vs. inappropriate use. At the population level, this measure may represent a setting's resource decision making and capacity to respond to the needs of people living with ADRD, as evidenced by settings with higher proportions of Medicaid residents associated with higher APU. State agencies can address the association of APU with organizational resources and (in)appropriate medication use as two different, though related, concerns.

$\mathrm{AL} / \mathrm{RC}$, as conceptualized, is a residential setting meant to support residents with health-related services and social engagement. ${ }^{116}$ Qualitative study and intentional collaboration with $\mathrm{AL} / \mathrm{RC}$ residents, care staff, operators, and care coordinators (external or onsite) is needed to contextualize how $\mathrm{AL} / \mathrm{RC}$ settings adapt to both the dynamic needs of their resident populations and resources required to meet those needs. Additional inter- and intrastate comparison of characteristics associated with APU, such as accounting for organizations that operate within and across state lines (ie, chains), could further elucidate how regulatory classifications and organizational characteristics impact delivery of quality care to $\mathrm{AL} / \mathrm{RC}$ resident populations. 


\subsubsection{Limitations \& Future Directions}

Though we examined data from 2017-2019 waves of the Oregon CBC study in this analysis, the associations reported necessitate additional data points for more precise estimates. The restricted focus of relevant structural characteristics related to care processes and outcomes on material and organizational conditions, and the lack of attention to the power structures that fundamentally dictate organizational characteristics, care processes, and outcomes of interest presents another limitation. ${ }^{269,270}$ Power structures take many forms, including interpersonal (eg, residents and care staff, care staff and supervisors, facility staff and corporate management), organizational (eg, $\mathrm{AL} / \mathrm{RC}$ settings, profit status, interaction with state agencies), socioeconomic (eg, access to additional services and primary care, ability to pay staff a living wage, pharmaceutical production and distribution), and structural (eg, capitalism, ableism, ageism, racism). For example, recent evidence also suggests increases in the prevalence of serious mental illness (eg, schizophrenia) in long-term care settings, ${ }^{271}$ conditions indicated for treatment with antipsychotic medications. However, a troubling trend is emerging where incident schizophrenia diagnoses in long-term care residents living with ADRD are increasing overall, with disproportionately higher rates among Black nursing home residents living with ADRD. ${ }^{272}$

Despite existing frameworks and best intentions, care quality conceptualization and measurement remains a social construct dependent on the social and political capital of those who decide upon the assessment criteria. ${ }^{273,274}$ Investigating how power dynamics influence decision making at interpersonal and systemic levels related to 
AL/RC residents' care would greatly strengthen future studies of long-term care populations.

The outcome measure-as operationalized in this study-does not fully capture the theoretical construct of APU, which encompasses several phenomena, including prescription, administration, production, and distribution. Prescription is the act of a prescriber (eg, medical doctor, nurse practitioner) writing an order for a medication as treatment for a condition. Prescriptions can be ordered on a scheduled or pro re nata (PRN; as needed) basis depending on the type of medication and the condition being treated. Administration describes the act of taking or being given medication. For staff to administer medications to residents, a prescription order is necessary. The presence of a prescription order, especially for PRN medications, does not necessarily equate to the administration of a medication. ${ }^{141}$ We only know the number of residents who received antipsychotic medication in the prior 90 days, not how many times an antipsychotic medication was administered nor the reasons for administration. Further upstream, the popularity of APU, particularly off-label use, is partially rooted in the costs and revenue associated with the production, distribution, and profit of these medications. ${ }^{17,275,276}$ External societal and economic environments frame the context of prescription and administration patterns, and warrant further study in the older adult population. While prevalence of APU is considered a quality metric in both nursing homes and assisted living, additional perspectives are needed to more holistically understand AL/RC settings' abilities to respond to residents' behavioral expressions, in addition to reducing the use of potentially inappropriate medications. 


\subsubsection{Conclusions \& Implications}

In Oregon, the 90-day prevalence of APU across licensed AL/RC settings is notably higher than what quality metrics would suggest is appropriate. Given state variation in $\mathrm{AL} / \mathrm{RC}$ regulation and oversight, understanding whether certain characteristics of $\mathrm{AL} / \mathrm{RC}$ settings are associated with higher or lower prevalence of APU can inform policy and practice development related to treating APU as a quality indicator. Policymakers, long-term care advocates, and $\mathrm{AL} / \mathrm{RC}$ organizations might consider how organizational resource capacity and meeting the needs of resident populations influence APU in AL/RC settings, especially if APU prevalence is treated as a quality indicator. Further understanding of medication administration and prescription decision making is necessary to contextualize reported rates of APU. 


\section{Appendix A. Supplemental Tables}

Table 2. Bivariate random intercepts regressions of organizational, process, and resident population characteristics associated with prevalence of antipsychotic use by setting type, 2017-2019

\begin{tabular}{|c|c|c|c|c|}
\hline \multirow[b]{2}{*}{ Survey Wave (ref. 2015-2016) } & \multicolumn{2}{|c|}{$\begin{array}{c}\text { AL/RC } \\
\beta[95 \% \mathrm{CI}]\end{array}$} & \multicolumn{2}{|c|}{$\begin{array}{l}\text { Any MCa } \\
\beta[95 \% \mathrm{CI}]\end{array}$} \\
\hline & & & & \\
\hline $2017-2018$ & -1.6 & {$[-4.8,1]$.} & -4.1 & {$[-10.0,1.8]$} \\
\hline 2018-2019 & -0.8 & {$[-2.6,-4.1]$} & -2.4 & {$[-9.0,4.1]$} \\
\hline \multicolumn{5}{|l|}{ Size (ref. $<25$ beds) } \\
\hline $25-49$ beds & $18.3^{-}$ & {$[-27.5,-9.1]$} & 0.4 & {$[-6.9,7.6]$} \\
\hline $50-74$ beds & $21.1^{* * *}$ & $\begin{array}{c}{[-29.5,-} \\
12.6]\end{array}$ & -0.4 & {$[-7.3,6.5]$} \\
\hline$\geq 75$ beds & $25.0^{* * *}$ & $\begin{array}{c}{[-33.2,-} \\
16.7]\end{array}$ & -2.8 & {$[-15.4,9.7]$} \\
\hline Rural/Frontier (ref. Urban) & -0.6 & {$[-5.6,4.3]$} & -0.3 & {$[-6.1,5.6]$} \\
\hline Nonprofit (ref. For profit) & -4.9 & {$[-11.3,1.5]$} & $-16.4^{* *}$ & {$[-25.9,-7.0]$} \\
\hline \multicolumn{5}{|l|}{ Medicaid census (ref. Low) } \\
\hline Moderate & 0.0 & {$[-5.1,5.2]$} & $11.9^{* *}$ & {$[4.8,19.0]$} \\
\hline High & $6.4^{*}$ & {$[1.5,11.2]$} & $13.6^{* * *}$ & {$[7.2,20.0]$} \\
\hline Very high & $13.1^{* * * *}$ & {$[6.6,19.7]$} & $13.4^{* *}$ & {$[5.6,21.2]$} \\
\hline Average age (years) & $-1.0^{* * *}$ & {$[-1.3,-0.6]$} & -0.5 & {$[-1.1,0.2]$} \\
\hline \multicolumn{5}{|l|}{ Proportion of residents: } \\
\hline Female & $-0.3^{* *}$ & {$[-0.5,-0.1]$} & -0.2 & {$[-0.4,0.0]$} \\
\hline Hispanic/Latino and/or POC ${ }^{b}$ & -0.0 & {$[-0.1,0.1]$} & 0.1 & {$[-0.1,0.3]$} \\
\hline Diagnosed with ADRD ${ }^{\mathrm{c}}$ & $0.2^{* * *}$ & {$[0.1,0.3]$} & - & - \\
\hline Diagnosed with SMI ${ }^{\mathrm{c}}$ & $0.7^{* * * *}$ & {$[0.5,0.8]$} & 0.2 & {$[-0.1,0.5]$} \\
\hline Assist w/ oral medications & 0.0 & {$[-0.0,0.1]$} & $0.2^{* * *}$ & {$[0.1,0.3]$} \\
\hline $\begin{array}{l}\text { Use hospice services (prior } 90 \\
\text { days) }\end{array}$ & $0.3^{*}$ & {$[0.1,0.6]$} & 0.3 & {$[-0.0,0.7]$} \\
\hline Behavioral needs index ${ }^{\mathrm{d}}$ & $0.6^{* * *}$ & {$[0.5,0.8]$} & 0.1 & {$[-0.0,0.3]$} \\
\hline$N$ (cluster) & \multicolumn{2}{|c|}{$598(297)$} & \multicolumn{2}{|c|}{$334(167)$} \\
\hline $\begin{array}{l}p<0.05,{ }^{* * *} p<0.01,{ }^{* * *} p<0.001 \\
\text { "Any MC" includes memory care units th } \\
\text { ettings. Because residents living in memo } \\
\text { le any MC model excludes ADRD due to } \\
\text { "POC" = people of color; Includes Ameri } \\
\text { ative Hawaiian/Pacific Islander, and mul } \\
\text { ADRD= Alzheimer's and related dement } \\
\text { Behavioral needs index }=[(\% \text { residents re } \\
\text { andering + \% danger to self/others }) / 3] \times\end{array}$ & $\begin{array}{l}\text { o-located } \\
\text { must have } \\
\text { arity. } \\
\text { an/Alaska } \\
\text { I-= seriou } \\
\text { assistanc }\end{array}$ & $\begin{array}{l}\text { ithin assisted liv } \\
\text { n Alzheimer's o } \\
\text { Jative, Asian, Bl } \\
\text { nental illness } \\
\text { for lack of awar }\end{array}$ & $\begin{array}{l}\text { g or resi } \\
\text { related d } \\
\text { k/Africa }\end{array}$ & $\begin{array}{l}\text { ntial care } \\
\text { entia diagnosis, } \\
\text { American, } \\
y+\%\end{array}$ \\
\hline
\end{tabular}




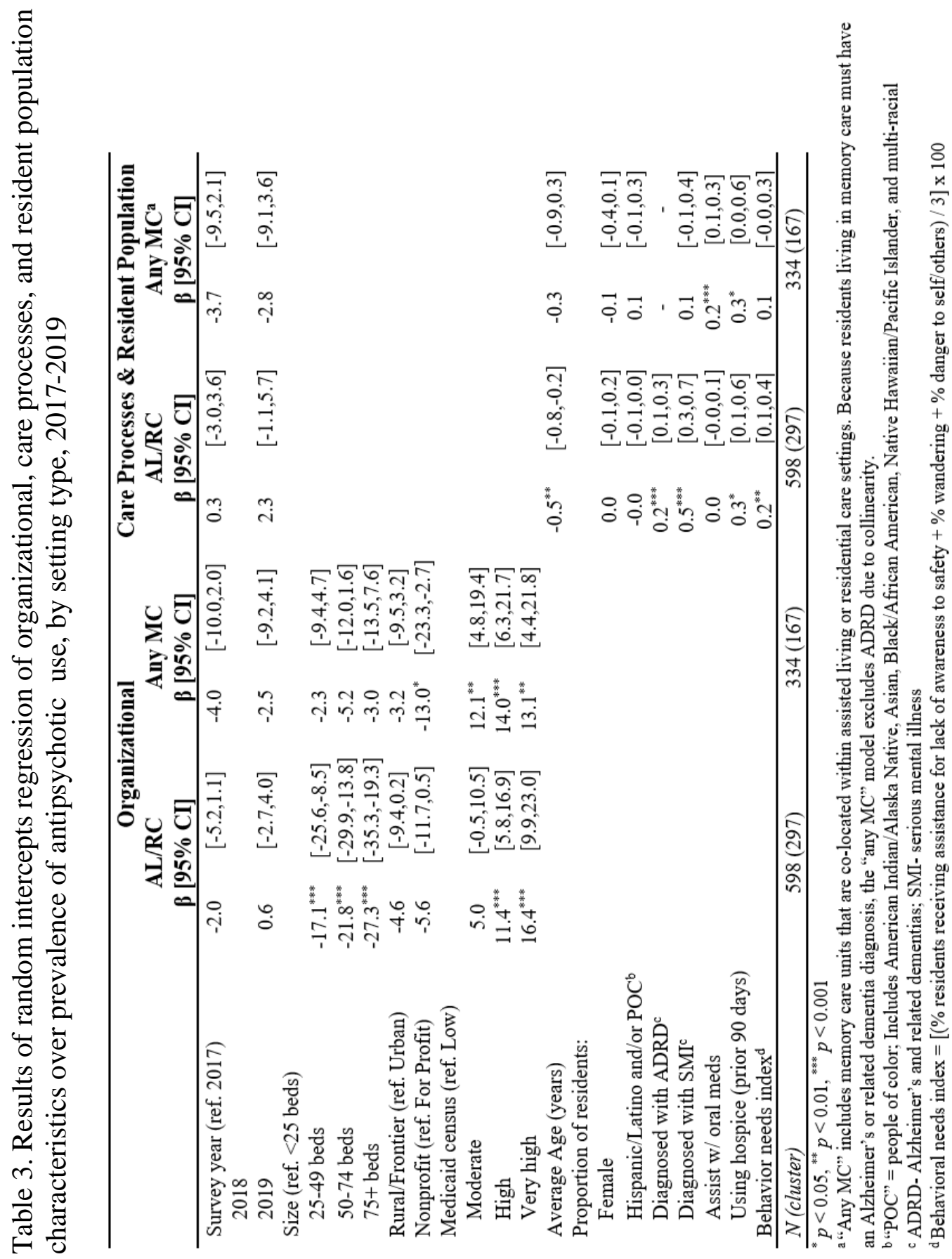


Table 4. Results of fully adjusted random intercepts regression models of organizational, care processes, and resident population characteristics over prevalence of antipsychotic use by setting type, 2017-2019.

\begin{tabular}{|c|c|c|c|c|}
\hline \multirow[b]{2}{*}{ Survey year (ref. 2017) } & \multicolumn{2}{|c|}{$\begin{array}{c}\text { AL/RC } \\
\beta[95 \% \text { CI }]\end{array}$} & \multicolumn{2}{|c|}{$\begin{array}{c}\text { Any MC } \\
\beta[95 \% \text { CI] }\end{array}$} \\
\hline & & & & \\
\hline 2018 & -0.2 & {$[-3.4,3.0]$} & -3.4 & {$[-9.2,2.4]$} \\
\hline 2019 & 2.0 & {$[-1.4,5.3]$} & -2.7 & {$[-9.2,3.7]$} \\
\hline \multicolumn{5}{|l|}{ Size (ref. $<25$ beds) } \\
\hline $25-49$ beds & -5.8 & {$[-11.9,0.4]$} & -2.4 & {$[-9.3,4.4]$} \\
\hline $50-74$ beds & -6.0 & {$[-12.2,0.2]$} & -6.5 & {$[-13.3,0.2]$} \\
\hline $75+$ beds & $-9.3^{* *}$ & {$[-15.8,-2.7]$} & 0.1 & {$[-10.3,10.4]$} \\
\hline Rural/Frontier (ref. Urban) & -1.6 & {$[-5.6,2.3]$} & -4.0 & {$[-9.8,1.9]$} \\
\hline Nonprofit (ref. For Profit) & $-4.4^{*}$ & {$[-8.4,-0.4]$} & $-12.4^{* *}$ & {$[-21.2,-3.6]$} \\
\hline \multicolumn{5}{|l|}{ Medicaid census (ref. Low) } \\
\hline Moderate & 1.8 & {$[-2.7,6.3]$} & $11.4^{* * *}$ & {$[4.6,18.2]$} \\
\hline High & $5.4^{*}$ & {$[0.4,10.5]$} & $12.8^{* * *}$ & {$[5.9,19.7]$} \\
\hline Very high & $8.9^{*}$ & {$[1.7,16.1]$} & $11.0^{*}$ & {$[2.3,19.8]$} \\
\hline Average Age (years) & -0.3 & {$[-0.6,0.0]$} & -0.1 & {$[-0.7,0.4]$} \\
\hline \multicolumn{5}{|l|}{ Proportion of residents: } \\
\hline Female & 0.0 & {$[-0.1,0.2]$} & -0.2 & {$[-0.4,0.0]$} \\
\hline Hispanic/Latino and/or POC ${ }^{b}$ & -0.1 & {$[-0.1,0.0]$} & 0.0 & {$[-0.2,0.2]$} \\
\hline Diagnosed with $\mathrm{ADRD}^{\mathrm{c}}$ & $0.2^{* * *}$ & {$[0.1,0.3]$} & - & - \\
\hline Diagnosed with $\mathrm{SMI}^{\mathrm{c}}$ & $0.5^{* * *}$ & {$[0.3,0.6]$} & 0.1 & {$[-0.2,0.4]$} \\
\hline Assist w/ oral meds & 0.0 & {$[-0.0,0.0]$} & $0.2^{* * *}$ & {$[0.1,0.3]$} \\
\hline Using hospice (prior 90 days) & $0.3^{*}$ & {$[0.1,0.6]$} & $0.3^{*}$ & {$[0.0,0.6]$} \\
\hline Behavior needs index ${ }^{\mathrm{d}}$ & $0.2^{*}$ & {$[0.0,0.4]$} & 0.1 & {$[-0.0,0.3]$} \\
\hline$N$ (cluster) & \multicolumn{2}{|c|}{$598(297)$} & \multicolumn{2}{|c|}{$334(167)$} \\
\hline \multicolumn{5}{|c|}{$\begin{array}{l}<<0.05,{ }^{* *} p<0.01,{ }^{* * *} p<0.001 \\
\text { "Any MC" includes memory care units that are co-located within assisted living or residential care settings. } \\
\text { Because residents living in memory care must have an Alzheimer's or related dementia diagnosis, the any MC model } \\
\text { xcludes ADRD due to collinearity. } \\
\text { "POC" = people of color; Includes American Indian/Alaska Native, Asian, Black/African American, Native } \\
\text { Hawaiian/Pacific Islander, and multi-racial } \\
\text { ADRD- Alzheimer's and related dementias; SMI- serious mental illness } \\
\text { Behavioral needs index = [(\% residents receiving assistance for lack of awareness to safety }+\% \text { wandering }+\% \\
\text { langer to self/others) / 3] x } 100\end{array}$} \\
\hline
\end{tabular}


Chapter 5: Beyond the Medication Pass: Attitudes, Ethics, Agency, and Antipsychotic Medications in Assisted Living/Residential Care

\subsection{Abstract}

Background: Pro re nata antipsychotic medication use (PRN APU) among assisted living/residential care $(\mathrm{AL} / \mathrm{RC})$ residents is a controversial health policy issue. AL/RC care providers, families, clinicians, researchers, and policymakers disagree about using these medications on an as-needed basis to manage behavioral expressions associated with residents' dementia or cognitive impairment.

Methods: Semi-structured interviews among 11 caregivers, medication technicians, nurses, administrators/executive directors, and consultant pharmacists currently working in Oregon AL/RC settings. I use situational analysis, an extension of grounded theory methods, to identify and describe positions and ideologies related to APU.

Findings: Situational analysis facilitated identification of three main themes. First, attitudes (ie, positive, neutral, and negative) inform whether to use PRN antipsychotic medications. Second, an underlying morality and positioning of PRN antipsychotics or nonpharmacologic interventions as "right" or "wrong" drive approaches to behavioral response. Finally, I found an inverse relationship between the perceived level of agency within and proximity to the situation of APU.

Conclusions: Participants described costs/benefits associated with PRN APU and nonpharmacologic interventions when responding to $\mathrm{AL} / \mathrm{RC}$ residents' behavioral expressions Understanding the scope and context of APU within AL/RC settings necessitates a broader systems-level approach to this issue. 


\subsection{Introduction}

Assisted living and residential care (AL/RC) is a fast-growing segment of the long-term services and supports sector in the United States (U.S.). ${ }^{52-54}$ Of the estimated $812,000 \mathrm{AL} / \mathrm{RC}$ residents in the U.S., 42\% have been diagnosed with Alzheimer's disease or related dementias (ADRD). ${ }^{1}$ Over $70 \%$ of AL/RC residents living with ADRD or cognitive impairment experience behavioral expressions. ${ }^{2}$ Behavioral expressions can include aggression, agitation, anxiety, delusions, hallucinations, and sleeplessness. ${ }^{5,8,57,59}$ Chronic or severe behavioral expressions have implications for residents' quality of life, family and caregiver burden, and care transitions. ${ }^{67,69,70,229,277}$

Nonpharmacologic and pharmaceutical interventions describe the two overarching approaches to responding to behavioral expressions. Existing guidelines and practices encourage psychosocial or environmental interventions as the first line of treatment in managing behavioral expressions as a person-centered, safe, alternative to medication. ${ }^{15,44,72,77,131,278}$ When nonpharmacologic interventions fail or a resident is in considerable, persistent distress, pharmaceutical management of behaviors using psychotropic medication may be appropriate ${ }^{77}$ Psychotropic medications interact with the central nervous system and include the following drug classes: antipsychotics, antidepressants, anxiolytics, hypnotics, and mood stabilizers. ${ }^{79,279}$

Though every psychotropic medication class is associated with significant risks in older adult populations, ${ }^{16,81,88,108,109,234,280,281}$ antipsychotic medications have received explicit empirical and regulatory attention. In the early 2000s, studies demonstrated that off-label use of antipsychotic medications in older adults with dementia was associated 
with a higher risk of early mortality, ${ }^{19,20}$ leading the U.S. Food and Drug Administration to issue a "black box" warning on antipsychotic medication use (APU) in older adults. ${ }^{93-}$ ${ }^{95}$ Given the prevalence of older adults living with dementia in long-term residential care settings, the Centers for Medicaid and Medicare Services developed quality improvement efforts related to the use of these medications, forming the National Partnership for Quality Dementia Care, ${ }^{25,104}$ which has been associated with reductions in antipsychotic medication use in $\mathrm{NH}$ populations over time. ${ }^{102,103,182,282}$ There is still lack of evidence regarding how antipsychotic medications are used, staff training and implementation of nonpharmacologic interventions, uptake of other psychotropic medications beyond antipsychotics, or potential discrimination against older adults whose behaviors are deemed "challenging" within the AL/RC context.

There are many reasons antipsychotic medications are prescribed and administered to older adults living with ADRD, primarily due to behavioral expressions labelled as agitation and aggression. ${ }^{10,283}$ Clinicians, caregivers, and family members reportedly view APU as positive, safe, and effective, ${ }^{29,30,101,284}$ though much of the existing literature only focuses on $\mathrm{NH}$ residents and staff. One qualitative study detailed how primary care providers primarily view pharmaceutical response to behavioral expressions associated with ADRD as less risky than empirical evidence suggests and that while policies do successfully decrease APU, they unintentionally promote other, riskier medications. ${ }^{30}$ The same study team found direct care staff and family caregivers in nursing homes described significant systemic and interpersonal barriers to performing nonpharmacologic interventions compared to the effectiveness of medications. ${ }^{29}$ Other 
literature has shown the paradox faced by caregivers regarding APU, recognizing that these medications are prescribed too frequently but can be the best of not ideal responses when working with older adults living with ADRD. ${ }^{99}$

Key factors that influence decision making around APU in nursing homes include organizational capacity, individual professional capability, communication and collaboration, attitudes, and regulations/guidelines ${ }^{285}$ In comparison to routine/scheduled use of these medications, pro re nata (PRN; as needed) medication orders to respond to behavioral expressions presents an additional layer of complexity and decision making, especially for direct care staff who are not permitted to formally assess residents' needs. ${ }^{78,141,199,286,287}$

Direct care staff (eg, caregivers, certified nursing assistants, and medication aides) build and maintain relationships with their residents, learning from and basing their care decisions on residents' cognitive, physical, and emotional cues on a daily basis. ${ }^{141,142,202,203}$ For those staff that assist with medications administered "as-needed," knowing individual residents' behaviors and nuances is critical to decision making around administration. ${ }^{139,142,143}$ Direct care staff may view behavioral and psychological expressions of dementia negatively, ${ }^{12}$ and have several strategies to address these behaviors, such redirection, isolation, seeking other coworkers' assistance, or communicated with external care providers. ${ }^{14,148,288} \mathrm{AL} / \mathrm{RC}$ care models that prioritize autonomy, choice, dignity, independence, individuality, and privacy introduce another component to the equation of medication management and APU. ${ }^{171,172,289}$ Balancing these espoused values (eg, autonomy) with the constraints of the care situation (eg, safety) can 
result in multiple sources and levels of conflict regarding an "appropriate response" to residents' behavioral expressions and medication needs. ${ }^{172,203,290-293}$

Antipsychotic medication prescription and administration in AL/RC settings is an under-studied and controversial community health and policy issue. Little is known about direct care staff and nurse's decision making and task-related responses to these behaviors. In this study, I explore decision making related to administering PRN antipsychotic medications among frontline $\mathrm{AL} / \mathrm{RC}$ staff: caregivers, nurses, administrators, and consultant pharmacists.

\subsection{Methods}

\subsubsection{Sampling and Recruitment}

A complete list of currently licensed AL/RC providers is publicly available through the state Department of Human Services website. ${ }^{204}$ Using this list, I selected facilities to contact using a random number generator until 25 administrators were identified. I then individually emailed a flyer explaining the purpose of my study and asking administrators to both participate in interviews and disseminate the flyer to their staff and coworkers (Appendix B). This flyer contained a link and QR code where individuals were provided with a digital consent form and could schedule a 30-minute interview with me on their own schedule. In addition to scheduling the interview, I asked participants to provide demographic information including their age, gender, race/ethnicity, and job title (Appendix C). Every seven to ten days I again emailed those who had not responded and repeated this process until enough participants responded to capture diverse experiences related to PRN APU. I offered participants a \$20 Amazon 
gift card for participating, with financial support from a private donor to the Portland State University Foundation for students studying gerontology. This study was approved by Portland State's Institutional Review Board (protocol \#: 206858-18).

\subsubsection{Data Collection}

Participants scheduled an interview time on their own providing me with their preferred method of contact, interviews took place over the phone and recorded and transcribed over Zoom. Participants provided additional, verbal consent to recording and then proceeded with the interview. I used a semi-structured interview approach, where I had an initial question bank (Appendix D). I began every interview with an introduction of the scope of the conversation and began with the following questions:

1) Please describe your job and what your responsibilities are.

2) Thinking about residents who have lived in this community who express behaviors, can you tell me about a time when one of those residents was helped through successful management by you and other staff?

3) How about a resident whose behavioral expressions were so severe that you or your staff were not able to respond, can you tell me that story?

I based the scenario questions (2 and 3) on a recent study examining staff reports of residents' behavioral expressions in AL/RC settings. ${ }^{10}$ How a participant described their roles and responsibilities guided which questions I followed up with from the available question bank. For example, a medication aide described medication passing as one of their roles, which led me to ask about their experiencing administering PRN antipsychotic medications. However, if an unlicensed caregiver indicated they did not 
have a role in medication administration, I did not ask about administering medications. The experiences participants chose to share also facilitated additional probes and follow up questions that arose within the interview.

Before closing the interview, I also asked every participant to share how a hypothetical policy would potentially affect their work. Specifically, "Hypothetically, if the state were to introduce a requirement that no more than $20 \%$ of assisted living, residential care, and memory care residents could have a PRN antipsychotic medication order, how would that affect your work?" I derived this question from existing guidelines for nursing home settings, ${ }^{104}$ and proposed quality goals for AL/RC settings. ${ }^{26}$ After downloading the recordings and transcripts to a secure cloud-based server, I listened to each recorded interview to ensure transcription accuracy.

\subsubsection{Data Analysis}

I used grounded theory methods for analyzing qualitative data. ${ }^{160,163-165,174}$ I read through each transcript and took notes on topics and patterns I noticed arising in each conversation. These formed the initial foundation of codes to describe PRN antipsychotic medication administration. I developed a preliminary set of a priori codes based on the interviewing experience and elements of situational analysis to orient myself to the data. General categories for codes included roles (eg, people, positions, jobs identified as involved), attitudes (eg, emotions, positions, thoughts related to antipsychotic medication and/or behavioral expressions), process (eg, descriptions of what leads to PRN antipsychotic medication administration), and proximity (eg, in relation to AL/RC residents and receiving antipsychotic medication, where are roles, attitudes, and process 
situated?). As an extension of coding practices aligned with a grounded theory approach, ${ }^{160,165}$ I implemented Adele Clarke's situational analysis, ${ }^{163,174-176}$ an approach that extends earlier theorists' (eg, Strauss, Corbin, Charmaz) work by incorporating pragmatic, constructivist and post-modern theories as well as cartographic mapping concepts. One purpose of situational analysis is to identify many possible interacting elements and characteristics related to a phenomenon of interest through a systematic series of visualization exercises, or mapping. ${ }^{163,174}$ The initial codes facilitated the visual analytic exercises described below and in the Findings section.

To investigate decision making related to as needed APU from the perspective of various caregivers within AL/RC settings, I used mapping exercises outlined by Clarke and her colleagues to extend and describe patterns among the initial codes I identified. ${ }^{173,175-177}$ Using analytic memos to reflect on the emergence of topics within the interviews and potential relationships among these topics, I began with "messy maps"163 for each interview (Figure 1). I used these messy maps to capture the breadth of topics, elements, and perspectives raised during interviews. Through an iterative process of analytic memoing I continued to integrate analytic and theoretical relationships, resulting in numerous maps.

The next phase of Clarke's situational analysis approach involves developing an ordered situational map to organize elements identified, during messy mapping, including those that might be unstated, or "silent." 
Figure 1. An example of a messy map constructed of interviewee \#9

Messy Map: Interview \#9, Residential Care Administrator

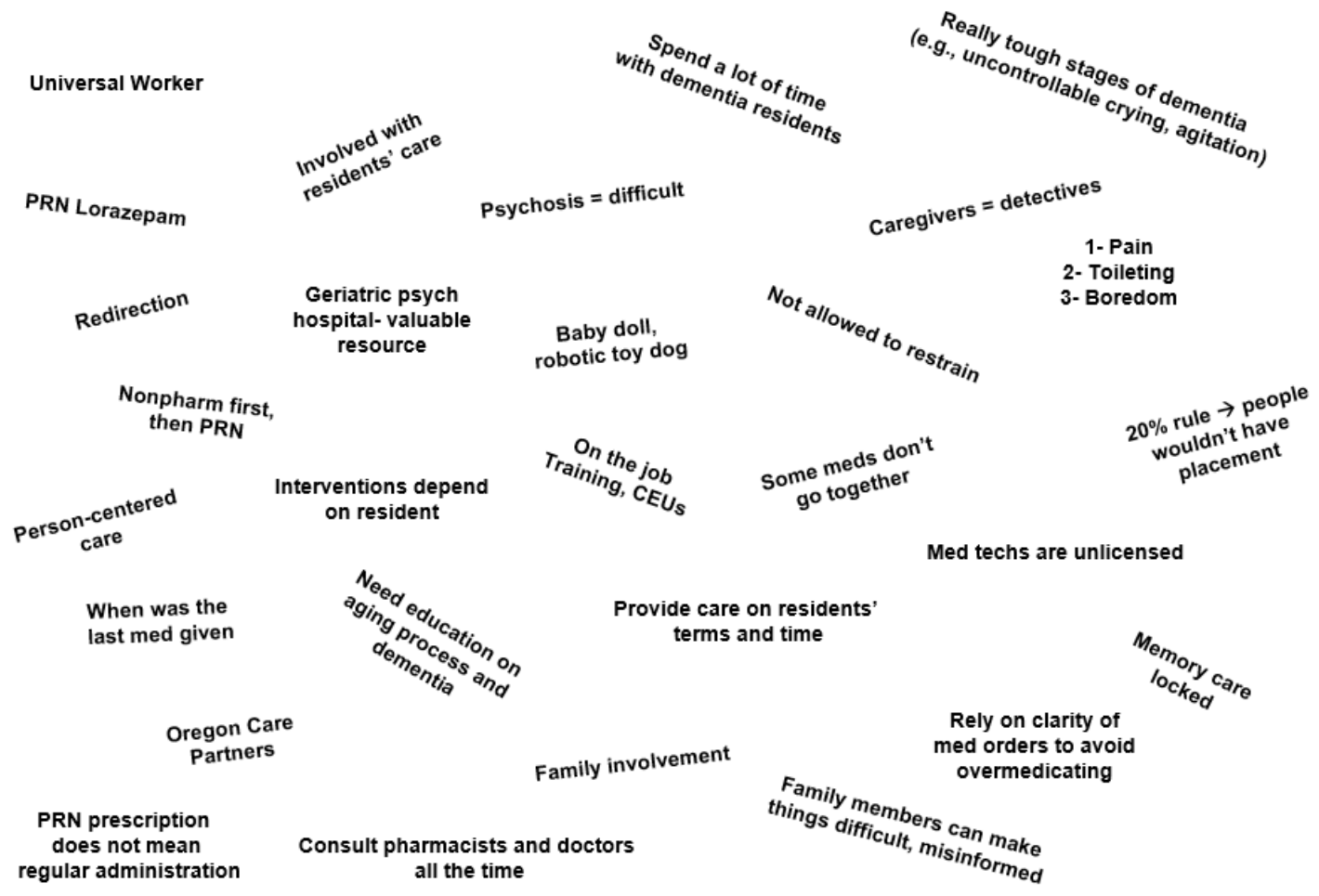

In my case, the goal was to identify elements that illustrate and contextualize the situation of PRN antipsychotic medication administration within assisted living based not only on interviews, but also the primary human (eg, residents, staff) and nonhuman (eg, PRN medications, medication records, order parameters, behaviors) elements involved in the situation, collective groups or organizations, discourses constructing human and nonhuman actors, political, sociocultural, temporal, historical, and other pertinent elements to the situation. Examples and definitions of these components are detailed in Appendix E. ${ }^{163,174}$ 
Participants described numerous human and nonhuman elements, identified positions and relationships among these actors and external political and social beliefs, and expressed varying positions and decision making related to PRN antipsychotic medication use. I use this ordered map in combination with line-by-line interview coding to develop themes illustrated in the Findings section. I developed these themes through intensive analytic memoing and positional maps. ${ }^{163,172,174}$ Using positions and relationships among human and nonhuman elements identified by participants, I plotted these descriptions along axes, using codes and quotes to visualize positions. These positional maps anchored thematic development and are supported with participants' quotes.

\subsection{Findings}

\subsubsection{Interview Participants}

I sent recruitment emails to 130 facilities from April to June 2021. Nineteen individuals indicated that they would participate in an interview, 16 consented and scheduled an interview, and 11 completed an interview. Table 1 describes the demographic characteristics of the interviewees, who included administrators/executive directors $(n=4)$, unlicensed direct care workers $(n=3)$, consultant pharmacists $(n=2)$, a licensed professional nurse $(n=1)$, and a registered nurse/resident care coordinator $(n=1)$.

Table 1. Descriptive characteristics of interview participants.

\begin{tabular}{|l|c|}
\hline Characteristics & Mean (range) or \% (n) \\
\hline Age in years & $44(27-64)$ \\
\hline Race/ethnicity & \\
\hline
\end{tabular}




\begin{tabular}{|r|c|}
\hline Non-Hispanic White & $64 \%(7)$ \\
\hline Asian American or Pacific Islander & $18 \%(2)$ \\
\hline Multiracial & $9 \%(1)$ \\
\hline Not disclosed & $9 \%(1)$ \\
\hline Woman & $64 \%(7)$ \\
\hline Man & $27 \%(3)$ \\
\hline Gender nonconforming & $9 \%(1)$ \\
\hline
\end{tabular}

5.4.2 Theme 1: Positive, negative, and neutral beliefs and attitudes justify as-needed antipsychotic medication utilization.

Interview participants described the use of PRN antipsychotic medications, types of related training, and how a hypothetical utilization threshold (ie, 20\% resident population) at the policy level would affect their work. When answering, participants conveyed different attitudes and beliefs regarding the use of as-needed antipsychotic medications in people with dementia living in AL/RC settings. One participant stated that "medication is an emotional, heavy topic" to discuss. Figure 2 depicts the range of attitudes expressed by interviews and their relationship with whether to use or not use antipsychotic medications.

Figure 2. Positional map of expressed ideologies on PRN antipsychotic medication use in assisted living/residential care residents with dementia. 


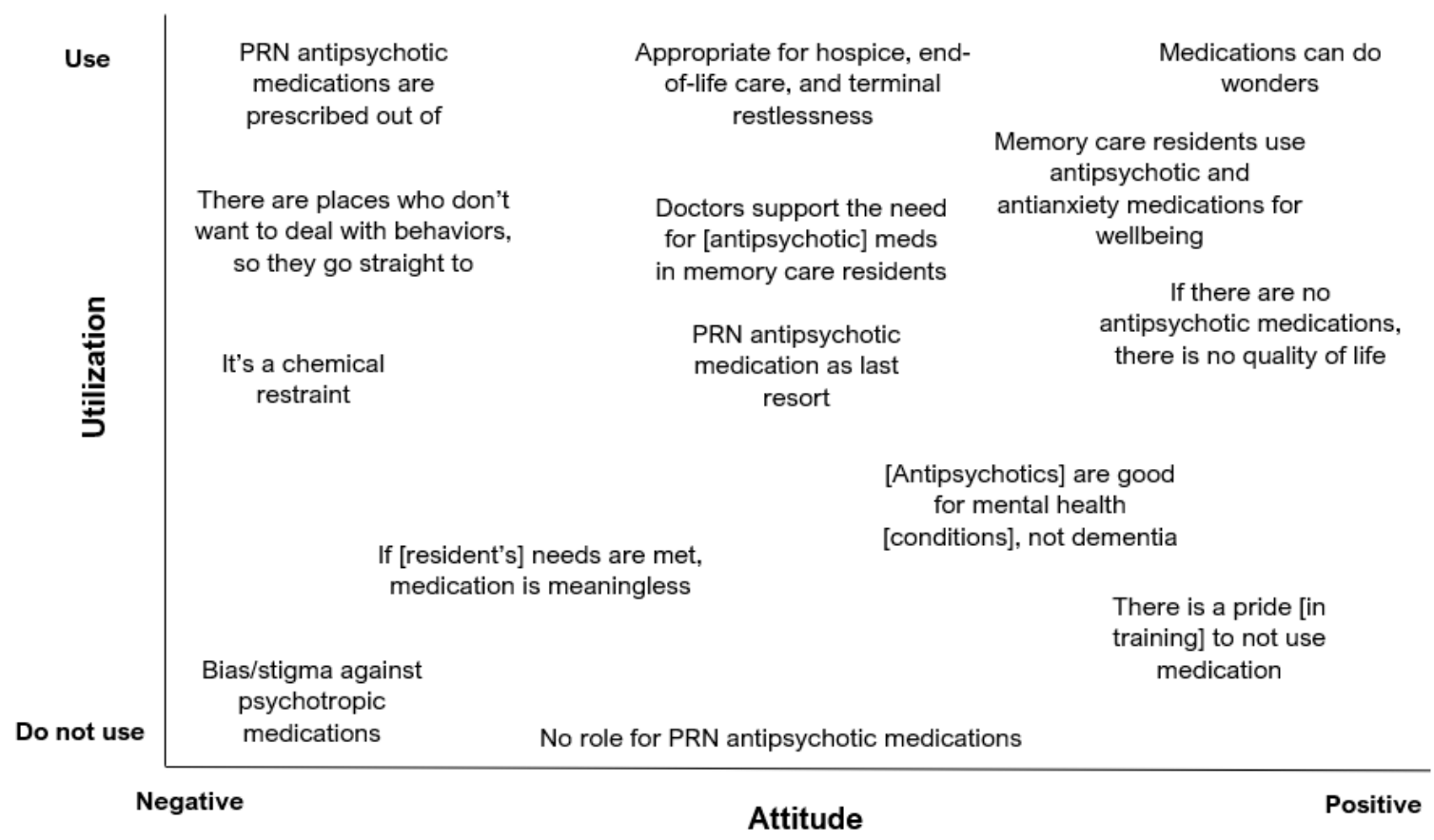

When describing examples of behavioral management and reflecting over the course their experiences working with residents, participants tended to describe whether it was appropriate to use or not use PRN antipsychotic medications. Participants underscored their comments and stories with positive, negative, and/or neutral attitudes regarding the use of antipsychotic medications.

Nearly every participant iterated in some capacity that medications are effective when residents exhibit behavioral expressions, pain, and/or other discomfort. Despite all efforts to address residents' needs and respond to behavioral expressions, residents may not respond to nonpharmacologic interventions. Several caregivers shared protocols and processes that guide behavioral response in the communities where they work. Often, after a number of unsuccessful nonpharmacologic interventions, they are guided by the 
residents' care plan and facility policy to escalate to medications as one medication technician described,

"[We use] at least three interventions usually laid out, like, planned interventions things that might work: snacks, toileting, repositioning. And then talking to the family about their [the resident's] past and getting ideas to redirect them. [...] But if at least three attempts don't work usually for the course of at least half an hour that's when we would consider giving them something stronger, some medication."

Though interview participants with direct care roles were asked to describe situations where they administered PRN antipsychotic medications to residents, few shared stories about antipsychotic medications, specifically. In their descriptions of the effectiveness of medications when working with assisted living, residential care, and memory care residents, interviewees were inclined to discuss PRN medications overall, which included other types of medications, "Yeah PRN, so you're talking about Hydrocodone, Oxycodone, Lorazepam, Morphine, you know?" When asked to describe a situation that necessitated administration of a PRN antipsychotic medication, nearly every participant (outside of consultant pharmacists) chose to tell a story about an antianxiety medication administration (eg, lorazepam).

Unlicensed caregivers, registered nurses, and administrators all shared the utility of medications when working with residents to promote comfort and quality of life. Two interviewees with nursing credentials (licensed professional nurse and registered nurse) shared that assisted living and residential care residents with PRN medication orders are able to self-direct and ask for those medications. However, for residents in memory care settings, 
"[...] a lot of the residents use antipsychotics and antianxiety [medications] because it's for their wellbeing and they're up, they're functional, they're going to activities, they have a good appetite, they're socializing, they're interested in things, you know? They're communicating to the best of their ability, they're definitely having a good quality of life, but if they didn't have those medicines they wouldn't be having a good quality of life."

Alternatively, some caregiving staff and those with pharmaceutical backgrounds stated antipsychotic (and other psychotropic) medications for as-needed use among residents living with dementia is not appropriate whatsoever. Rather, using these types of medications functions as a restraint and indicates residents' needs are not being met. A consultant pharmacist who works with communities to conduct resident medication review said,

"These drugs are all indicated to treat schizophrenia and various psychiatric disorders, but when we're using them to treat dementia, they don't do anything in dementia, they don't slow its progression. We're essentially using a chemical restraint. And sometimes that's necessary somebody that has exacerbating behaviors and is explosive and hitting everybody, kicking scratching basically the neuropsychiatric symptoms of dementia, we don't really have anything to treat those symptoms, so we default to antipsychotics. You use them basically tranquilizers."

Overall, despite the job role, interviewees shared that PRN APU has appropriate and inappropriate uses for assisted living residents. A combination of clinical decision making, resident-driven care planning, and appropriate staffing were all cited as facilitators to making an appropriate decision whether to administer PRN antipsychotic medications or not.

5.4.3 Theme 2: Responding to a resident's behavioral expression with or without medication is partially driven by an underlying morality/ethic. 
When participants were asked questions about responding to resident behaviors, either successfully or unsuccessfully, several individuals raised the premise of "responding right" or "responding wrong." Regarding the use of antipsychotic medications or nonpharmaceutical approaches, participants acknowledged that responses should ideally center individual residents and their needs. However, respondents described ethical toggle when deciding to use nonpharmaceutical intervention versus a PRN antipsychotic medication. This toggle was framed in several ways (Figure 3). Generally, respondents oriented their perspectives to "medication as a last resort" through broadly applied nonpharmaceutical interventions or individually designed interventions. In one community, a medication technician described typical nonpharmaceutical responses residents' behavioral expressions:

"We have some residents with dementia and they yell, they scream, so sometimes they are hungry, we want to offer them snacks, you know? Maybe their briefs are wet, so we need to change [their briefs]. Or sometimes there's some resident like to watch TV, so there's loud music, you know, some people doesn't want to hear it, so we need to change the, you know, place. Like, we need to bring [them to] their room, or we need to bring some things in and turn down the music, you know, like that."

Some respondents detailed specific interventions that were developed for individual residents based on specific behaviors. One respondent described a memory care resident who would often attempt to elope from the secured unit:

"One woman, she ran dog shows, and so, she would travel to different parts of the country and put on dog shows, and there would be awards, and all of that, and so, she would believe oftentimes in the afternoons that she had to get out of here so that she could go catch a plane for a dog show somewhere. So her care plan listed specific things to say to her, because she was worried she was going to miss her plane, and so we would say, 'Oh, my gosh I forgot to tell you it's been rescheduled until tomorrow. You're going to get your flight in the morning." 
Figure 3. Positional maps of perceived morality associated with response to assisted living/residential care residents' behavioral expressions.

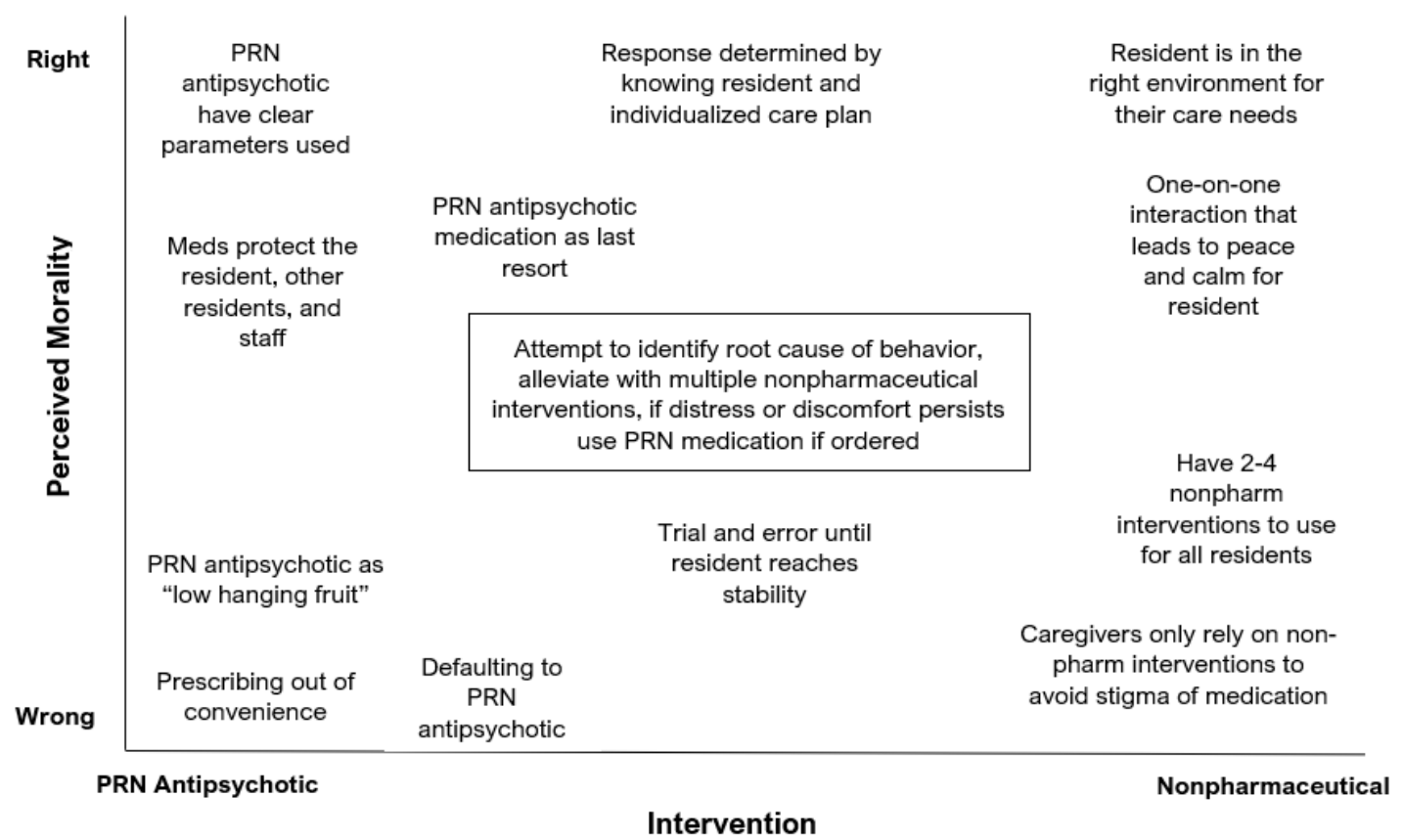

Notes. Text in the center box describes the prescribed rule via regulations and recommended practice.

Other respondents shared that despite all best efforts, sometimes "medications just work" and one can "try everything" and the resident will remain in distress. The same respondent above followed the description of the resident who ran dog shows in a previous life with a caveat and concern for the personal and professional preference towards nonpharmaceutical interventions, associating too many with negative consequences,

"So lots of times we use, most times we use, nonpharmaceuticals. My personal concern is that, having been in long term care since '93, I see a lot of non-licensed staff who are taught to believe that there's a concern that they're overmedicating people or using those psychotropics for their convenience. And that bias that is built into them results in them trying too many non-pharmaceuticals, resulting in poor outcomes for our folks." 
Interview participants tended to describe multiple perspectives over the course of their interviews. Nearly every participant started by describing an overarching position regarding the right or wrong of medications vs. nonpharmacologic interventions. Concurrently, participants also justified decisions or situations that may be opposite or contradictory to their primary view.

For example, a consultant pharmacist stating there is no role for PRN antipsychotic medications and following with a caveat that hospice or end-of-life care presents an exception to this rule,

"Honestly there's really no role for PRN antipsychotics, there are very rare circumstances. You know, there's instances, maybe end-of-life care terminal restlessness. In general, for [PRN] antipsychotics, we call them low hanging fruit, we need to get rid of those. There's very rare situations that we should use [them]."

\section{Subtheme 2a: Appropriate vs. inappropriate use of as needed antipsychotic} medications framed by "good guy" vs. "bad guy" mentality. Several participants indicated they had either worked at settings or heard of settings who abuse PRN medications. One executive director of a memory care setting shared the complexity around the issue of as-needed antipsychotic medication use among residents. They said,

"It's a really touchy subject because I am sure there are some places out there who overmedicate and they will do that because they don't want to deal with the behaviors, which is a huge disservice to our people. So finding that balance is super important, you know you always hear the bad guys ruin it for the good guys. That's true, because a lot of these policies out there, they don't allow you to use them as they should be, in the correct manner, because people abuse it. But we are not all bad I promise you." 
Perceptions of goodness also extend to trust in and beliefs about clinical providers and prescribers responsible for overseeing medication management. A caregiver speculated on the reasoning behind using medication to respond to behaviors, questioning whether it is appropriate,

"Since the pill almost seems like a restraint, or I don't want to say a punishment, but it's like 'we can't handle you [resident] anymore, so we need you to take this pill, so we don't have to, you know, deal with you' is kind of what it feels like. But it can't be that way right? Because there's all these caring people, the administrator is really nice, the doctor is really nice, the nurse is really nice."

One nurse described their perceptions of prescriber's intentions and knowledge related to prescribing as-needed antipsychotic medications to residents, suggesting this treatment modality is both well-intentioned and informed,

"The people who are ordering the medications, I'm assuming they're all good people and they wouldn't just order things negligently, but I can say that they are aware enough of the pros and cons of antipsychotics."

\subsubsection{Theme 3: Proximity to medication administration is inversely related to perceived} agency/authority.

Participants discussed the complexity surrounding PRN antipsychotic medication administration. By the time direct care staff administer a PRN antipsychotic medication to an assisted living resident, several actors with varying credentials, familiarity, and proximity to the situation and the resident have made a number of decisions. The multilayered nature of PRN antipsychotic medication administrations suggests different power dynamics and ability to participate in the situation (Figure 4). As one participant shared, 
'It [antipsychotic medication use] is really a prescribers' issue. The facility doesn't write the prescription. So the onus is on the facility to try to get gradual dose reduction or at least get a response and there's a lot of physicians that just won't respond. If you ever really wanted to change, you'd have to mandate some sort of physician training like they had to do with opioids or something like that. Yeah and probably get the state Board of Medical Examiner's involved in mandating some sort of specialized CME [continuing medical education] or something."

Within the situation of medication administration typically exists the resident receiving the medication and the staff member administering the medication. Participants described different levels of agency depending on a) residents' level of cognitive impairment and ability to communicate and b) staff's roles within the settings. One caregiver described their observations and resulting questions when giving residents with behaviors medications,

"The pill almost seems like a restraint, or I don't want to say a punishment, but it's like where we can't handle you anymore, so we need you to take this pill, so we don't have to deal with you. [...] They give me a pill after [a resident] does these behaviors and then this is the outcome after [the resident] takes the pill, just there in [their] chair, you know? So like all the stuff that's in my mind as a caregiver, and I don't have the authority to say anything, or if I did have questions how would I address them or take them to my administrator or my nurse?"

This participant expressed feeling disempowered to voice their concerns and ask questions by virtue of their position as an unlicensed direct caregiver. Participants with medication technician roles tended to focus on the responsibility of medication administration,

"At night I'm basically the acting supervisor. I make sure that all of the tasks are done and the caregivers are getting their jobs done as well as doing whatever scheduled medications there are and PRN medications, often helping with end of life comfort." 
Participants also raised the importance of knowing and building relationships with residents to facilitate quality care and implement best practices. Staff turnover, low staffing ratios, and low perceived agency among staff within assisted living facilities compared to external providers were commonly expressed obstacles related to meeting residents' needs and responding to behavioral expressions.

Many participants expressed both appreciation and frustration with existing and proposed regulations and policies. While appreciating the purpose of regulations to protect the safety and wellbeing of residents as assisted living consumers, participants feel those in the position to enact rules and requirements are disconnected from the practice of providing care to residents,

"It is something that you wish, you know, the rule makers in the legislature and up at DHS [Department of Human Services] knew about the work that you do when they're setting regulations. They know they can't know every building in the whole state of Oregon, but it would be nice if they would ask for more information about the populations we deal with and what kind of difficulties we have."

State regulations set the minimum standards expected of facilities when providing care and services to residents. Though, rule makers and those in positions to create and amend these regulations are removed from the daily rhythm within facilities. Though not explicitly raised in interviews, other service providers external to medication administration within AL/RC settings (eg, social workers, psychiatric providers) might have input and agency related to APU. 
Figure 4. Positional map of perceived agency and proximity to PRN antipsychotic administration in assisted living/residential care settings.

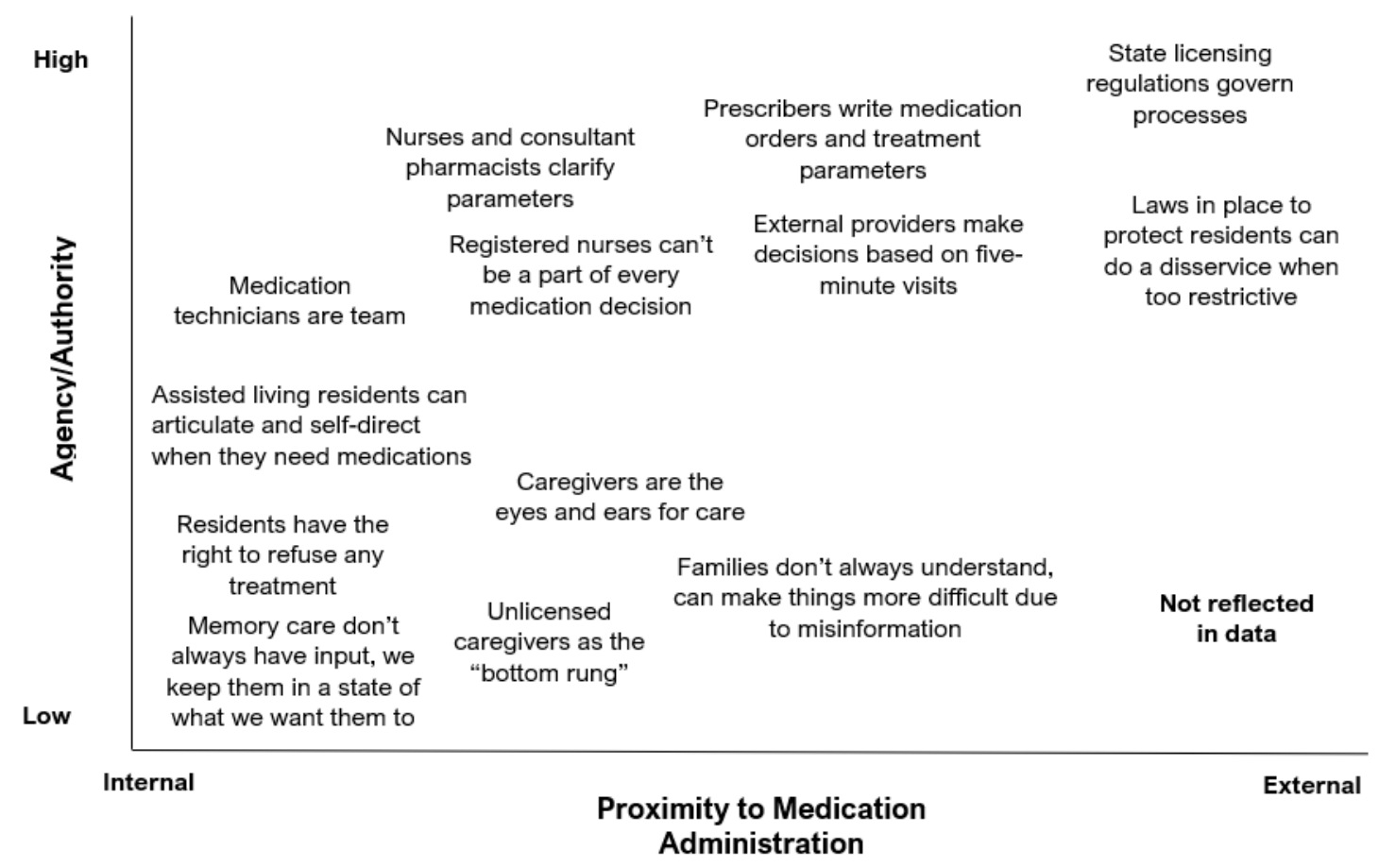

\subsection{Discussion}

In this study, I explored how those within the AL/RC setting with direct care connection and medication oversight of $\mathrm{AL} / \mathrm{RC}$ residents, including direct care staff, medication aides, administrators, and consultant pharmacists, make decisions regarding PRN APU. By extending grounded theory practice with a situational analytic approach, I found that attitudes (ie, positive, negative, and neutral), an underlying morality guiding nonpharmacologic/pharmaceutical interventions, and perceived agency within the context of medication administration influence study participants' ideologies around PRN APU. These findings highlight the complexity underlying the issue of APU in AL/RC settings 
and build upon narratives of medication management phenomena in the assisted living context. $^{139,172,200,203}$

The finding that positive, negative, and even neutral attitudes frame participants' beliefs around whether to use antipsychotic medications to respond to assisted living residents' behaviors is largely confirmatory. Like Kerns and colleagues found in their studies, ${ }^{29,30,101,283}$ participants with positive attitudes cited antipsychotic medication use as largely effective and promote wellbeing, especially for residents living with dementia. Related to the findings presented by Gill and colleagues, ${ }^{99}$ participants' views related to PRN APU situated along a positive/negative binary. A pattern emerged between job roles and whether participants were more likely to express positive or negative attitudes towards APU. At one end of the spectrum, unlicensed caregivers and consultant pharmacists described how using PRN antipsychotic medication to respond to residents' behaviors is effectively a chemical restraint for settings with staff that "don't want to deal with them [residents].” On the opposite end of the spectrum, medication technicians, nurses, and administrators were more likely to frame APU as promoting resident quality of life and wellbeing. Evaluations of the Halting Antipsychotic use in Long-Term care (HALT) study suggest that staff type plays a role in influencing success or failure of antipsychotic medication deprescription. ${ }^{286,294}$ Registered nurses and family members were described as drivers of represcribing of antipsychotic medications, especially in response to behaviors labelled as agitation and aggression. ${ }^{286}$ Combined with the context of one's job or role within $\mathrm{AL} / \mathrm{RC}$, how participants conceptualize and perceive dementia and dementia care needs can influence care decisions. ${ }^{295-297}$ 
Dementia care involves systems of thought and belief that guide decisions about what is "good" and "bad" (ie, morals) and what might be "right" or "wrong" to do (ie, ethics). ${ }^{298,299}$ Further, implications of and ethical issues with off-label use of antipsychotic medications to manage behavioral expressions have been discussed across the life course and globe. ${ }^{199,300-306}$ This conversation is further complicated by whether actors' motivations align or conflict. An oversimplified example might manifest as clinicians pursuing therapeutic goals (eg, symptom management), families concern with safety goals, direct care staff prioritizing resident-centered goals, and administrative staff might prioritize compliance goals. ${ }^{100,287,298,303,305}$

Participants' experiences highlighted an underlying morality that partially drove whether to approach residents' behavioral expressions with PRN antipsychotic medication or nonpharmacologic interventions. Participants detailed examples of when it is "right" or "wrong" to use either PRN antipsychotic medications or nonpharmacologic interventions. Based on Oregon Administrative Rules Chapter 411, Division 54, Section 6, when responding to resident behaviors, staff must first attempt nonpharmacologic interventions and document whether they are effective. If ineffective, and the resident remains in distress, PRN psychotropic medications may be considered..$^{77,92,122}$ Major sources of moral distress for formal caregiving staff include understaffing, perceiving residents with dementia in pain, not having enough time to provide adequate care. ${ }^{307}$

Some participants raised concerns with providing care that might be "regulationcentered" as opposed to resident-centered, resulting in unintended consequences. ${ }^{308}$ Fear of regulatory noncompliance might lead staff to attempt too many behavioral 
interventions, prolonging a resident's pain or distress or putting staff or other resident's in harm's way. ${ }^{31}$ Participants shared that doing what is best for the resident should drive decision making, even if what is perceived as "best" includes elements of deception (eg, white lies). ${ }^{309,310}$ However, resource constraints and organizational obstacles present significant barriers to the one-on-one person centered approach that is often required, and recommended as best practice. ${ }^{13,72,311,312}$ Other studies have reported similar experiences across licensed and unlicensed care staff, where organizational and systemic barriers deprioritize implementation of nonpharmacologic interventions. ${ }^{14,29,313,314}$

Lastly, participants situated their decision-making within perceived agency and authority. The human actors that participate in the situation of medication administration within AL/RC settings vary in proximity (ie, internal vs external) and power within the situation. In this study, I found an inverse relationship between the perceived authority of an entity or individual and their proximity to medication administration with AL/RC settings. At the heart of medication administration within AL/RC are the resident receiving the medication and the staff person administering the medication. Prior studies have focused in on the intersection of resident and staff autonomy with regard to balancing safety, wellbeing, and choice regarding medication management in AL/RC. ${ }^{139,141,172,203}$ AL residents have the right to refuse any treatment per resident rights recognized in regulatory practice, ${ }^{122}$ but the ability to assert this autonomy is largely influenced by cognitive capacity, care needs, staff perceptions of residents' abilities, and AL/RC setting culture. ${ }^{172,203,290,315,316}$ Participants remarked on the difference in 
articulating needs and requesting medication by residents living in memory care settings compared to those in assisted living and residential care.

Long before a medication aide administers a PRN antipsychotic medication to a resident, other entities outside of the $\mathrm{AL} / \mathrm{RC}$ setting context have made numerous decisions, and direct care staff must work within the parameters presented to them. ${ }^{312}$ In this study, I found that entities or individuals assigned with the most authority (ie Department of Human Services rule makers, physicians). Nurses and administrators oversee the writing of medication order parameters, ensuring direct care staff can administer medications and treatments without making assessment decisions. Prescribers write the original orders and generate access to the medications within AL/RC settings. Pharmacists or nurses review medication orders, recommending changes within the context of clinical decisions and regulatory compliance. Additionally, residents' families present another human element with varying degrees of power over their loved one's care, depending on their level of involvement and legal authority (eg, legal guardian, health proxy). Understanding the scope and context of APU within AL/RC settings necessitates a broader systems-level approach to this issue - beyond the medication pass.

\subsubsection{Limitations \& Future Directions}

This study has several limitations worth considering for future research efforts. First, the ongoing COVID-19 pandemic presented significant barriers to recruitment and sampling for this study, affecting participant selection into the study. Long-term care settings, including assisted living, have been disproportionately impacted by both resident 
morbidity and mortality and staffing shortage burdens as a direct result of COVID19. ${ }^{317,318}$ In addition to this trauma, pandemic-related restrictions limited recruitment and data collection efforts to fully remote, which adversely affected my ability to build the trust and relationships necessary with frontline care staff and administrators to gain buyin for this research. Losing the ability to recruit participants onsite required me to rely on administrators to disseminate information during a period of heightened stress and burnout. ${ }^{319}$ Future research teams interested in conducting interviews or focus groups with the $\mathrm{AL} / \mathrm{RC}$ workforce should consider investing in strategies that prioritize building relationships over the long term with administrators and staff and collaborate on data collection designs that simultaneously mitigate burden and offer an opportunity for participants to share their experiences.

There were only 11 participants in this study, spread across different facilities and the state of Oregon. These participants delivered rich, deep interviews, but it is possible key experiences involving PRN APU are missing based on who had the capacity to participate and restricting the sample to those with connection to care and medication oversight within the AL/RC setting. This study would have been greatly strengthened by interviewing more staff who worked in the same facility and with the same resident population to aid contextualization of decision making around PRN antipsychotic medication use. Relatedly, this study only captures the views of direct care staff, nurses, administrators, and consultant pharmacists. Though participants illustrated connections and importance of other actors involved in medication administration, resident, family member, and prescriber perspectives are missing from the narrative presented in this 
study. Future research studies could move upstream and attempt to map out external mechanisms that influence PRN antipsychotic medication administration, particularly the relationships and situations that arise among residents, their families, and prescribers.

\subsubsection{Conclusions \& Implications}

This study raises practice and policy implications regarding APU in AL/RC settings. Roles related to caregiving, ethical considerations, and perceived agency inform decision making on whether to use antipsychotic medications. Participants described costs and benefits associated with both PRN APU and nonpharmacologic interventions when responding to AL/RC residents' behavioral expressions. Participants' experiences emphasize the interactions across multiple levels of care (eg, interpersonal to policy level). Balancing regulatory goals and norms with resident-centered practices underscores the need for a systems-level perspective, extending beyond direct care staff passing antipsychotic medications to residents. 
Appendix B. Recruitment Flyer

\section{DIRECT CARE STAFF! \\ Do you help residents with their medications in this community? \\ We want to learn from you!}

My name is Sarah Dys, I am a Community Health student at Oregon Health \& Science University-Portland State University School of Public Health. I am interested in learning how and why antipsychotic medications are used in assisted living, residential care, and memory care settings. I am looking for administrators, nurses, care staff, and pharmacists who work in this community to participate in an anonymous 15-30-minute interview because you have direct, daily experience caring for residents. This research study is not an evaluation of you or this community.

\section{You will receive a \$20 Amazon gift card for participating!}

If you are interested in participating in an anonymous interview, please use your phone to scan the following QR code or visit this link:

\section{https://tinyurl.com/antipsychstudy}

Thank you for your time! If you have questions about this

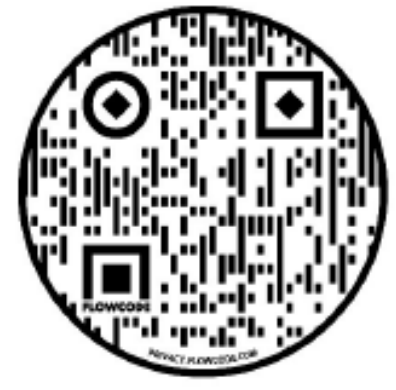
study, please contact:

Sarah Dys (978) 606-1033 sdys@pdx.edu

Paula Carder (503) 725-5411 carderp@pdx.edu 


\section{Appendix C. Text from Recruitment Questionnaire and Consent Form for Potential Interview Participants}

Welcome! Thank you for your interest in participating in a research study.

\section{Why is this research being done?}

Antipsychotic medication use and administration in AL/RC settings is a public policy priority in Oregon from a safety, oversight, and quality perspective. Quality Metrics Council members have raised concerns that the goal of reducing antipsychotic medication use will result in unintended consequences that may harm individuals with serious mental illness, or result in residents with behavioral expressions being transferred out of AL/RC settings.

We are asking you to participate in a research study because of your experience working with assisted living, residential care, and/or memory care residents. It is important to learn about your perspectives and experiences. We are looking to interview administrators, nurses, consultant pharmacists and direct care staff to learn about your experiences, decision making, and training regarding antipsychotic medication use. This is not an evaluation of yourself, your staff, or your community.

Please continue to the next page to review additional information about the study and read the consent form.

\section{Consent to Participate in Research}

Download a copy of the following consent form:

https://drive.google.com/file/d/1pHjny1n5nIvdwIbrxviYkrZPipwdmiK/view?usp=sharing

You are being asked to take part in a research study. The bullet points below highlight the main information about this research for you to consider when deciding whether or not to join in the study. Please carefully look over the information given to you on this form. Please ask questions about any of the information you do not understand before you decide to agree to take part. 


\section{Key Information for You to Consider}

- Voluntary Consent. You are being asked to volunteer for a research study. It is up to you whether you choose to take part or not. There is no penalty if you choose not to join in or decide to stop your involvement.

- Why is the study being done? The reason for this research is to learn how and why antipsychotic medications are used in assisted living, residential care, and memory care populations in Oregon.

- How long will it take? Your participation will take a total of 15 to 30 minutes over the course of one interview. However, the length of the interview is dependent on how much information you are willing to share.

- What will I be expected to do? You will be asked to participate in a virtual or phone interview scheduled at your convenience. The researcher will ask you to respond to a scenario you might see at work and follow up with a few questions about how you make decisions and what you do in certain situations. With your permission, the researcher will record your responses on a recording device.

- Risks. Some of the possible risks or discomforts of taking part in this study include breach of confidentiality, becoming upset, tired, frustrated, or distracted.

- Benefits. You will receive a \$20 gift certificate for your participation in this study. Additionally, the study may help to increase knowledge which may help others in the future.

- Options. Participation is voluntary and the only alternative is to not participate.

What happens to the information collected? Information collected for this research will be summarized in a report that will be shared to stakeholders such as advocacy organizations, state policymakers, assisted living provider groups as well as published in academic journals. Any information collected during this study and that can be linked to you or identify you will be kept private and confidential. No identifiable information, such as your name or the name of where you work, will be used in any papers or publications resulting from this study. No information about you will be shared with the administrator or management here; this study is not an evaluation of your work.

How will I and my information be protected? We will take measures to protect your privacy including using a codename when referring to you in study materials, and keeping any materials with identifying information (such as this form) in a password protected folder that can only be accessed with two-step verification on a virtual private network at Portland State University. Despite taking steps to protect your privacy, we can never fully guarantee that your privacy will be protected. 
To protect all your personal information, we will not use your real name when recording interviews or share any information regarding your participation with the administrator, supervisors, other staff, or residents. Despite these precautions, we can never fully guarantee that all your study information will not be revealed.

Individuals and organizations that conduct or monitor this research may be permitted access to inspect research records. This may include private information. These individuals and organizations include the Institutional Review Board that reviewed this research and Dr. Paula Carder, the director of the Institute on Aging at Portland State University.

What if I want to stop my part in this research? Your part in this study is voluntary. You do not have to take part in this study, but if you do, you may stop at any time. You have the right to choose not to take part in any study activity or completely stop at any point without penalty or loss of benefits to which you are otherwise entitled. Your decision whether or not to join in will not affect receiving the gift card, or your relationship with the researchers or Portland State University.

Will it cost me money to take part in this research? There is no cost to taking part in this research, beyond your time.

Will I be paid for being in this research? You will receive a $\$ 20$ gift card for participating in this research.

Who can answer my questions about this research? If you have questions, concerns, contact the research team at:

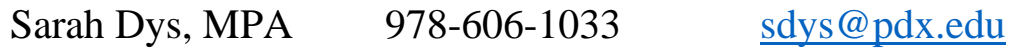

Paula Carder, PhD 503-725-5144 arderp@pdx.edu

Who can I speak to about my rights as a part of research? The Portland State University Institutional Review Board ("IRB") is overseeing this research. The IRB is a group of people who independently review research studies to ensure the rights and welfare of participants are protected. The Office of Research Integrity is the office at Portland State University that supports the IRB. If you have questions about your rights, or wish to speak with someone other than the research team, you may contact:

Office of Research Integrity

PO Box 751

Portland, OR 97207-0751
Phone: (503) 725-5484

Toll Free: 1 (877) 480-4400

Email: psuirb@pdx.edu 


\section{Consent Statement}

I have had the chance to read and think about the information in this form. I have asked any questions I have, and I can make a decision about my participation. I understand that I can ask additional questions anytime while I take part in the research.

Please state one of the following:

$\square \quad$ I agree to take part in this study

- I do not agree to take part in this study

- If participant consents, move to next section.

- If participant does not consent, move to end of form.

1. What is the name of the assisted living, residential care, or memory care community where you work? If you work at more than one community, please indicate how many communities you work for.

2. How many hours do you work on a typical week?

3. What is your current position job title?

a. Direct care staff- No health license or certification

b. Direct care staff- Certified Medication Aide/Technician

c. Direct care staff- Certified Nursing Assistant

d. Licensed vocational/professional nurse (LPN/LVN)

e. Registered nurse (RN)

f. Resident Care Coordinator/Health and Wellness Director

g. Pharmacist/Consultant Pharmacist

h. Administrator/Executive Director

i. Other:

4. What is your age?

5. Which race/ethnicity/ities do you identify as? Please select all that apply.

a. African American/Black

b. American Indian/Native American/Alaska Native

c. Asian/Asian American/South Asian

d. Hispanic/Latinx

e. Native Hawaiian or Pacific Islander

f. White

g. Other:

h. Prefer not to say

6. How do you identify?
a. Woman
b. Man
c. Transgender woman
d. Transgender man
e. Nonbinary 


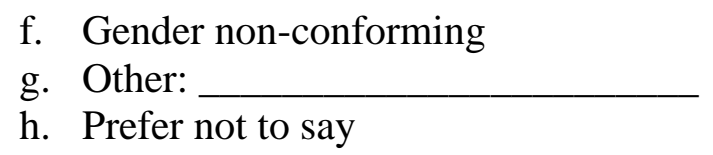

Please use the following link to schedule your interview.

https://calendly.com/sdys/antipsychotic-use-in-oregon-assisted-living

We will use the email address you provide here to send you your \$20 Amazon gift certificate.

End of Form.

Thank you so much for your time and participation! I look forward to speaking with and learning from you! 
Appendix D. Interview guide

Introduction: Older adults living with Alzheimer's disease and related dementias are more likely to express certain behaviors. When living in an assisted living or residential care environment, it is often up to caregiving staff to respond to resident's behaviors. You may be aware that antipsychotic medication use and administration in AL/RC settings is a public policy priority in Oregon from a safety, oversight, and quality perspective.

There is a lot of talk about antipsychotic medications, but we would like to know more about your experiences working with older adults in assisted living.

Can you start by telling me a little bit about your job and what your responsibilities are?

1. Thinking about residents who have lived in this community who express behaviors, can you tell me about a time when one of those residents was helped through successful management by you and other staff?

2. How about a resident whose behavioral expressions were so severe that you or your staff were not able to respond, can you tell me that story?

\section{FOLLOW UP QUESTION BANK}

- How do you know if an intervention works or does not work when responding to residents' agitation, anxiety, or distress?

- What types of training have you had on medication administration in older adults?

- Please describe a situation when you administered a PRN antipsychotic medication.

- How did you decide to administer this antipsychotic medication? Was there anything you would have done differently?

- What procedures or protocols does your facility/community/company have on record for the use of antipsychotic medications?

- If a resident has an order for an antipsychotic medication, what are you looking for when reviewing their Medication Administration Record (MAR)?

- How has the COVID-19 pandemic affected medication administration at this community?

- Let's imagine the state proposes a rule that no more than $20 \%$ of residents can have an as needed antipsychotic medication. How would that affect your work? 


\section{Appendix E. Ordered situational map of participant interviews}

\begin{tabular}{|c|c|c|c|c|c|}
\hline $\begin{array}{l}\text { Individual Human } \\
\text { Elements/Actors } \\
\text { e.g., key individuals and } \\
\text { significant (unorganized) people } \\
\text { in the situation }\end{array}$ & \multicolumn{4}{|c|}{$\begin{array}{l}\text { Nonhuman Elements/Actants } \\
\text { e.g., technologies; material infrastructures; specialized information and/or knowledges; material } \\
\text { "things" }\end{array}$} & \multirow[t]{2}{*}{$\begin{array}{l}\text { Collective Human } \\
\text { Elements/Actors } \\
\text { e.g., particular groups; specific } \\
\text { organizations }\end{array}$} \\
\hline Medication technician & Challenging behavior & \multicolumn{3}{|c|}{ Staff: resident ratios $\quad$ Creativity } & \\
\hline Psych provider & Scheduled medications & \multicolumn{3}{|c|}{ Clinical decision making $\quad$ Teamwork } & Staff (generally) \\
\hline Administrator & PRN medications & \multicolumn{2}{|c|}{ Chronic mental illness } & Communication & Health providers/clinicians \\
\hline Resident(s) & Dosage & \multicolumn{2}{|c|}{$\begin{array}{l}\text { Teepa Snow's Positive } \\
\text { Approach to Care }\end{array}$} & $\begin{array}{l}\text { Bias/Stigma around } \\
\text { medication use }\end{array}$ & non-licensed staff \\
\hline Primary care provider & Peace & \multicolumn{2}{|c|}{ Regulations } & Comfort & Board of Directors \\
\hline Prescriber & Calm & \multicolumn{2}{|c|}{ Social model of care } & Distress & Relias \\
\hline Consultant pharmacist & $\begin{array}{l}\text { Nonpharmacologic } \\
\text { intervention }\end{array}$ & \multicolumn{2}{|c|}{ Quality measures } & Progress notes & Consonus Pharmacy \\
\hline $\begin{array}{l}\text { Caregiver/universal } \\
\text { worker }\end{array}$ & Urinary tract infection & \multicolumn{2}{|l|}{ Citations } & $\begin{array}{l}\text { Family involvement } \\
\text { (or lack thereof) }\end{array}$ & Oregon Health Authority \\
\hline Surveyor & stability & \multicolumn{2}{|l|}{ Indications } & Ethics & Oregon Health Care Association \\
\hline Nurse & person-centeredness & \multicolumn{2}{|l|}{ Dementia } & $\begin{array}{l}\text { Relationship } \\
\text { building }\end{array}$ & $\begin{array}{l}\text { Oregon Partnership for Dementia } \\
\text { Care }\end{array}$ \\
\hline $\begin{array}{l}\text { Licensed professional } \\
\text { nurse }\end{array}$ & staff turnover & \multicolumn{2}{|c|}{$\begin{array}{l}\text { Neuropsychiatric } \\
\text { symptoms }\end{array}$} & $\begin{array}{l}\text { Managerial } \\
\text { constraints }\end{array}$ & CMS \\
\hline Social worker & $\begin{array}{l}\text { Medication administration } \\
\text { policy }\end{array}$ & \multicolumn{2}{|c|}{ Gradual dose reduction } & Antipsychotic & Corporate long-term care \\
\hline Mental health worker & PRN parameters & \multicolumn{2}{|c|}{$\begin{array}{l}\text { Medication } \\
\text { administration record }\end{array}$} & Benzodiazepine & FDA \\
\hline Police & Care/service planning & \multirow{4}{*}{\multicolumn{2}{|c|}{$\begin{array}{l}\text { Chemical restraint } \\
\text { FDA black box warning } \\
\text { Alcohol/substance use } \\
\text { Fear } \\
\text { Worry }\end{array}$}} & Antidepressant & American Medical Association \\
\hline Medic/EMTs & Facility/community culture & & & Psychotropics & Oregon Care Partners \\
\hline Resident's Spouse/Child & Documentation/charting & & & Narcotics & Crisis teams \\
\hline & $\begin{array}{l}\text { Care transitions } \\
\text { Training/Education }\end{array}$ & & & $\begin{array}{l}\text { Opioids } \\
\text { Pain }\end{array}$ & Department of Human Services \\
\hline \multicolumn{6}{|c|}{$\begin{array}{c}\text { Discursive Construction of Human Actors } \\
\text { As found in the situation }\end{array}$} \\
\hline \multicolumn{3}{|c|}{ Need to know residents as people } & \multicolumn{3}{|c|}{$\begin{array}{l}\text { So used to resident behaviors we become complacent, charting does } \\
\text { not always reflect experience }\end{array}$} \\
\hline \multicolumn{3}{|c|}{ Trying to make it person-centered } & \multicolumn{3}{|c|}{$\begin{array}{l}\text { Providers give multiple PRNs for the same issue, how are unlicensed } \\
\text { staff supposed to distinguish }\end{array}$} \\
\hline \multicolumn{3}{|c|}{$\begin{array}{l}\text { Pharmacist role to tease out when medicine is really necessary, goal } \\
\text { is to reduce }\end{array}$} & \multicolumn{3}{|c|}{ Medication techs as team leaders/acting supervisors } \\
\hline \multicolumn{3}{|c|}{ PCPs and prescribers generally lack geriatric knowledge } & \multicolumn{3}{|c|}{ Giving medications is a big responsibility } \\
\hline \multicolumn{3}{|c|}{$\begin{array}{l}\text { In dementia, behaviors wax and wane, prescribers don't want to } \\
\text { reduce these medications because of perceived stability }\end{array}$} & \multicolumn{3}{|c|}{$\begin{array}{l}\text { Doctors are knowledgeable, know what they are doing and acting in } \\
\text { best interests of residents }\end{array}$} \\
\hline \multicolumn{3}{|c|}{ A lot of staff don't have the background to be a med aide } & \multicolumn{3}{|c|}{ Caregivers as the "eyes and ears" / "detectives" } \\
\hline Staff have a tough job to do & & & $\begin{array}{l}\text { AL reside } \\
\text { and requ }\end{array}$ & $\begin{array}{l}\text { s can vocalize, tell you } \\
\text { t medications. Memory }\end{array}$ & $\begin{array}{l}\text { their needs, when needs are met, } \\
\text { care is different }\end{array}$ \\
\hline $\begin{array}{l}\text { Assumption that if resident } \\
\text { out of control }\end{array}$ & did not have antipsychotic, th & ey would be & Residents & feelings are valid, neec & to be listened to \\
\hline Who can self-direct and wh & o cannot? & & $\begin{array}{l}\text { Resident } \\
\text { other way }\end{array}$ & exhibit behaviors becat & use they can't express emotions in \\
\hline nurse responsible for medic & ation administration training & & RN can't & a part of every decisic & \\
\hline $\begin{array}{l}\text { "There are people who wou } \\
\text { residents" }\end{array}$ & Id rather give a pill than deal & & Caregiver & as family & \\
\hline $\begin{array}{l}\text { Doctors order medication } \mathrm{s} \\
\text { ensuring residents are in th }\end{array}$ & $\begin{array}{l}\text { the person fits the setting ra } \\
\text { e right setting for their needs }\end{array}$ & ther than & Unlicense & caregivers as the "bot & tom rung" \\
\hline Need to know residents as & people & & "Keep res & lents in a state of what & we want them to be" \\
\hline Trying to make it person-ce & ntered & & Are peop & with ADRD allowed to & display their disease? \\
\hline $\begin{array}{l}\text { Pharmacist role to tease ou } \\
\text { is to reduce }\end{array}$ & t when medicine is really nec & essary, goal & Residents & espond to "vibes of ca & regivers and other residents" \\
\hline Some nurses go immediate & Iy to medication, others wait & & & & \\
\hline
\end{tabular}




\begin{tabular}{|c|c|c|c|}
\hline \multicolumn{4}{|c|}{$\begin{array}{l}\text { Discursive Construction of Nonhuman Actants } \\
\text { As found in the situation }\end{array}$} \\
\hline \multicolumn{2}{|l|}{ Facility as home } & \multicolumn{2}{|c|}{ Medication record software in $\mathrm{AL}$ is not as robust } \\
\hline \multicolumn{2}{|l|}{ Care transitions as triggering } & \multicolumn{2}{|c|}{ No medications to effectively treat NPS } \\
\hline \multicolumn{2}{|c|}{ Overmedication vs. undermedication } & \multicolumn{2}{|c|}{$\begin{array}{l}\text { Facilities that do it right have people wandering, engaged, and not } \\
\text { falling. }\end{array}$} \\
\hline \multicolumn{2}{|l|}{ Finding a balance } & \multicolumn{2}{|c|}{$\begin{array}{l}\text { Antipsychotics are used so commonly in memory care that you would } \\
\text { think they are indicated to treat dementia and they are not }\end{array}$} \\
\hline \multicolumn{2}{|c|}{ Medications should be used correctly } & \multicolumn{2}{|c|}{ Meeting resident needs increases care quality } \\
\hline \multicolumn{2}{|l|}{ Medication as last resort } & \multicolumn{2}{|c|}{ Clear medication orders prevent overmedicating } \\
\hline \multicolumn{2}{|c|}{$\begin{array}{l}\text { Regulations have unintended consequences; skilled settings are } \\
\text { seeing unintended consequences from regulations that were } \\
\text { supposed to help }\end{array}$} & \multicolumn{2}{|c|}{ Laws prevent staff from abusing residents } \\
\hline \multicolumn{2}{|c|}{$\begin{array}{l}\text { Lot of work to determine why medications are started and determine } \\
\text { root causes of behavior }\end{array}$} & \multicolumn{2}{|l|}{ Can of worms } \\
\hline \multicolumn{2}{|c|}{ Assisted living lacks regulations and data } & \multicolumn{2}{|c|}{ Some places abuse meds and residents by zonking them out } \\
\hline \multicolumn{2}{|c|}{ Really clear parameters help non-nurses } & \multicolumn{2}{|l|}{ Assisted living is a gray area } \\
\hline \multicolumn{2}{|c|}{$\begin{array}{l}\text { Staffing is a primary barrier to person-centered care because one-on- } \\
\text { one interventions take time. }\end{array}$} & \multicolumn{2}{|c|}{$\begin{array}{l}\text { Antipsychotics are indicated for psychiatric disorders such as } \\
\text { schizophrenia, they don't do anything for dementia and operate as a } \\
\text { chemical restraint }\end{array}$} \\
\hline \multicolumn{4}{|c|}{ Interdisciplinary team approach to CBC } \\
\hline \multicolumn{4}{|c|}{ Medication as weighted, emotional topic } \\
\hline \multicolumn{4}{|l|}{ Every situation is different } \\
\hline \multicolumn{2}{|c|}{$\begin{array}{c}\text { Political/Economic Elements } \\
\text { e.g., the state; particular industry/ies; local/regional/global orders; political } \\
\text { parties; NGOs; politicized issues }\end{array}$} & \multicolumn{2}{|c|}{$\begin{array}{c}\text { Sociocultural/Symbolic Elements } \\
\text { e.g., religion; race; sexuality; gender; ethnicity; nationality; logos; icons; other } \\
\text { visual and/or aural symbols }\end{array}$} \\
\hline $\begin{array}{l}\text { One on one interventions take } \\
\text { time and money }\end{array}$ & $\begin{array}{l}\text { Desire for minimum state requirement } \\
\text { for staffing }\end{array}$ & $\begin{array}{l}\text { Who gets included in care } \\
\text { planning decisions? }\end{array}$ & $\begin{array}{l}\text { Positioning of the self- what would I } \\
\text { want }\end{array}$ \\
\hline $\begin{array}{l}\text { Generic Haldol or Ativan is } \\
\text { pennies per tablet, much } \\
\text { cheaper than hiring more } \\
\text { staff, maximize returns }\end{array}$ & $\begin{array}{l}\text { Not allowed to restrain residents, } \\
\text { meds seem like a restraint }\end{array}$ & Clinician approach & $\begin{array}{l}\text { Whose comfort and distress are at } \\
\text { the center of decision making? }\end{array}$ \\
\hline $\begin{array}{l}\text { Facilities are penalized but } \\
\text { facilities do not write } \\
\text { prescriptions. }\end{array}$ & $\begin{array}{l}\text { Can't force someone to take } \\
\text { something they don't want to }\end{array}$ & Medical toolkit & Avoid giving PRN to avoid stigma \\
\hline $\begin{array}{l}\text { AstraZeneca fined } \$ 600 \\
\text { million for pushing Seroquel } \\
\text { for sleep }\end{array}$ & $\begin{array}{l}\text { Laws in places to protect elders can } \\
\text { do a disservice when they are too } \\
\text { restrictive }\end{array}$ & $\begin{array}{l}\text { Comparison to nursing } \\
\text { facilities }\end{array}$ & $\begin{array}{l}\text { Exhaust all options before giving } \\
\text { med }\end{array}$ \\
\hline $\begin{array}{l}\text { Can't have one person caring } \\
\text { for } 25 \text { people at } \$ 12 / \text { hour }\end{array}$ & $\begin{array}{l}\text { If there was a regulation tying } \% \text { use } \\
\text { of antipsychotics to quality, who is in }\end{array}$ & $\begin{array}{l}\text { Memory care should not be } \\
\text { "nice and quiet" }\end{array}$ & What does behavior mean? \\
\hline $\begin{array}{l}\text { CMS guidelines do not apply } \\
\text { to physicians }\end{array}$ & $\begin{array}{l}\text { charge of the extra scrutiny, } \\
\text { paperwork, and heartache? }\end{array}$ & Consistent, right care team & $\begin{array}{l}\text { Strong medications are not good for } \\
\text { health }\end{array}$ \\
\hline $\begin{array}{l}\text { Regulations/rules come from } \\
\text { a safety point of view, rules } \\
\text { can be hard to follow in } \\
\text { practice }\end{array}$ & $\begin{array}{l}\text { If there was a regulation tying \% use } \\
\text { of antipsychotics to quality, who is in } \\
\text { charge of the extra scrutiny, } \\
\text { paperwork, and heartache? }\end{array}$ & $\begin{array}{l}\text { Using more medications than } \\
\text { normal is a sign that resident } \\
\text { needs are not being met. If } \\
\text { needs are met, behaviors go } \\
\text { away }\end{array}$ & $\begin{array}{l}\text { the care conference: who is involved, } \\
\text { who is not involved, identify goals of } \\
\text { care, how to accomplish, and identify } \\
\text { risks/benefits }\end{array}$ \\
\hline $\begin{array}{l}\text { Implementing a \% threshold } \\
\text { would impact }\end{array}$ & & $\begin{array}{l}\text { Trust between care team- } \\
\text { resident-health provider }\end{array}$ & Meds as protection \\
\hline $\begin{array}{l}\text { admissions/intake decisions; } \\
\text { change the definition of ability } \\
\text { to meet care needs. Go from }\end{array}$ & & $\begin{array}{l}\text { Root causes, why are } \\
\text { residents acting the way they } \\
\text { do? }\end{array}$ & $\begin{array}{l}\text { Handle situation by treating } \\
\text { residents as human beings }\end{array}$ \\
\hline regulation centered & & $\begin{array}{l}\text { Bias/stigma related to } \\
\text { psychotropic medication use }\end{array}$ & $\begin{array}{l}\text { Qualification of unlicensed caregivers } \\
\text { to have an opinion on this issue }\end{array}$ \\
\hline & & & $\begin{array}{l}\text { "Different and bad sometimes mean } \\
\text { the same thing" }\end{array}$ \\
\hline
\end{tabular}







Chapter 6: Synthesis of Research

This dissertation investigated how and why antipsychotic medications are used in AL/RC settings, focusing in on the Oregon policy and practice landscape. Through specific aims, I explored the following research questions:

1) What is the scope of psychotropic medication-related survey deficiency citations in Oregon AL/RC settings;

2) Are organizational characteristics of $\mathrm{AL} / \mathrm{RC}$ settings associated with antipsychotic use prevalence?; and

3) How do care AL/RC staff make decisions about antipsychotic medication administration in Oregon AL/RC settings?

I designed this study with the intention of bringing together multiple data sources, perspectives, and methods to comprehensively describe the situation of APU within Oregon's AL/RC population. The perspectives reflected in this study in regulatory oversight, $\mathrm{AL} / \mathrm{RC}$ resident population within the state, and experiences of a selection of care staff and providers who have a role in providing care or overseeing medication management in AL/RC.

\subsection{Main Takeaways}

Across the three studies undertaken in this dissertation, I found a combination of confirmatory evidence as well as novel contributions to the empirical discussion of APU in older adults living with ADRD and in AL/RC settings. In the section, I summarize the main findings across the three research studies. 
In Chapter 3, I analyzed narrative descriptions of deficiency citations issued to licensed AL/RC settings in Oregon from 2015 to 2019. Specifically, I analyzed citations issued for noncompliance with Oregon Administrative Rule 411-054-055-06, Psychotropic Medication. Similar to other studies, ${ }^{194,196}$ I found that most deficiencies were issued because of errors with documentation. The difference in severity between recordkeeping errors and abuse (ie, chemically restraining residents) is important to consider in combination with the relatively low prevalence of psychotropic medications issued (170 over a four-year period). However, documentation discrepancies or missing information does have implications for care provision. Lack of documenting implementation and effectiveness of nonpharmacologic interventions and how individual residents express their behaviors was compounded by lack of role clarity and specificity of medication administration parameters. Additionally, it is unclear based on the data included for this study how AL/RC settings implement plans for correction and the types of assistance provided by the DHS/APD to facilitate improvement. These findings raise questions regarding the balance, or lack thereof, between regulatory expectations and the practicalities of caregiving and operating an AL/RC setting.

Chapter 4 offers a state-level perspective on APU within Oregon's AL/RC population. Other studies examining factors associated with rates of APU are conducted at the individual-level with access to residents' medication administration records and/or electronic health records. ${ }^{16,127,132,133,231,280,281}$ In prior work, we found that AL/RC residents living in MC units, who had a serious mental illness or diagnosed with ADRD were more likely to receive an antipsychotic medication in the prior 7-day period. ${ }^{134}$ 
However, patterns of association seen among individual $\mathrm{AL} / \mathrm{RC}$ residents might not be replicated when comparing settings of AL/RC residents. ${ }^{320,321}$ Assuming that higher proportions of residents with ADRD or serious mental illness will show a similar magnitude of effect on the rate of APU within AL/RC settings is an individualistic fallacy. ${ }^{322,323}$ One conclusion in line with this fallacy might look like the following: to lower the rate of APU in AL/RC settings in Oregon, we must reduce the proportion of residents with ADRD or serious mental illness in any given AL/RC setting.

The findings demonstrated in Chapter 4 indicate that at the setting-level organizational characteristics are associated with larger effects on higher or lower APU rates among $\mathrm{AL} / \mathrm{RC}$ settings. In particular, $\mathrm{AL} / \mathrm{RC}$ settings with a very high prevalence of Medicaid users (67.8\%-100\%) reported average APU rates nine percentage points higher than AL/RC settings with low Medicaid use (0\%-15\%). Our analysis of individual residents did not find a significant relationship among individual residents' Medicaid status and APU. ${ }^{134}$ This suggests that APU at the setting-level is partially affected by an organization's resource capacity—having a higher proportion of residents using Medicaid reimbursement to pay for services compared to private pay has been associated with higher rates of APU. ${ }^{256,259,265}$

Lastly, Chapter 5 takes dives into the experiences and positions of those providing care to and overseeing medications for $\mathrm{AL} / \mathrm{RC}$ residents, including unlicensed caregivers and medication aides, nurses, administrators/executive directors, and consultant pharmacists. Using in-depth, semi-structured interviews, I explored participants’ experiences with responding to residents' behavioral expressions, administering PRN 
antipsychotic medications, and thoughts on a hypothetical policy related to limiting PRN APU. Through situational analysis, I used mapping techniques to elicit patterns and themes within the narrative data. ${ }^{176}$ I found that individual perceptions and attitudes, ethics of care, and perceived agency related to APU influence participants' ideologies and decisions to use pharmaceutical or nonpharmacologic approaches to behavior response.

For interpretive ease, the various positions described in Chapter 5 are visualized along two-dimensional axes. This oversimplification might suggest participants' positionality, experiences, and approaches fall explicitly along binary spectrums. In reality, participants shared multiple perspectives over the course of their interviews. Every participant expressed how deciding whether to use a PRN antipsychotic medication relied on numerous factors, situations, and contexts for each individual resident. This individualization is one of the values underpinning the AL/RC model, ${ }^{324}$ and has been demonstrated in the context of health care needs. ${ }^{153,154}$ However, AL/RC values, regulatory oversight, and resident-centeredness might not align cohesively in practice. ${ }^{325}$ Regarding PRN APU in Oregon AL/RC residents, providers and caregivers are forced to simultaneously balance and prioritize regulatory goals, organizational constraints, and complex care provision resulting in a multilayered, difficult, and unique situation.

\subsection{Social World of Antipsychotic Medication Use in AL/RC}

I operationalize elements of the Institutional Analysis and Development framework $^{36,150}$ by using situational analysis ${ }^{163}$ to contextualize the findings of the three 
studies undertaken in this dissertation. The elements of the framework described in section 2.7 informed the initial study design and approach to this dissertation study. These included exogenous variables such as material conditions (eg, organizational and environmental characteristics), community characteristics (eg, Oregon AL/RC resident population characteristics), and rules (eg, Oregon Administrative Rules Chapter 411, Division 54, training requirements, compliance guidelines).

I focused on the operational level of analysis, or the action situation, ${ }^{150}$ in this dissertation. Figure 1 presents the social world of APU in Oregon AL/RC settings through the lens of the dissertation findings. I recognize it is not possible to comprehensively cover every possible action and outcome that may occur. Social world maps embrace the inherent "messiness" of complex situations, highlighting the possibilities for actions and decisions across relevant elements, such as human actors, nonhuman elements, discourses, and sociopolitical elements). ${ }^{163,174,177,326}$

Across the entire situation of APU in Oregon AL/RC settings, I present major actors' subworlds, ${ }^{169,170}$ in interconnected circles: residents, families, AL/RC staff, consultant pharmacists, primary care providers, regulatory entities (state and federal), pharmaceutical industry, and long-term care research. APU in AL/RC populations is the intersection of health policy, practice, and research. The focused attention on off-label antipsychotic medications in older adults living with ADRD was birthed in the realm of research and manifested through policy decisions (ie black box warning, quality metrics). ${ }^{19,20,93-95}$ 


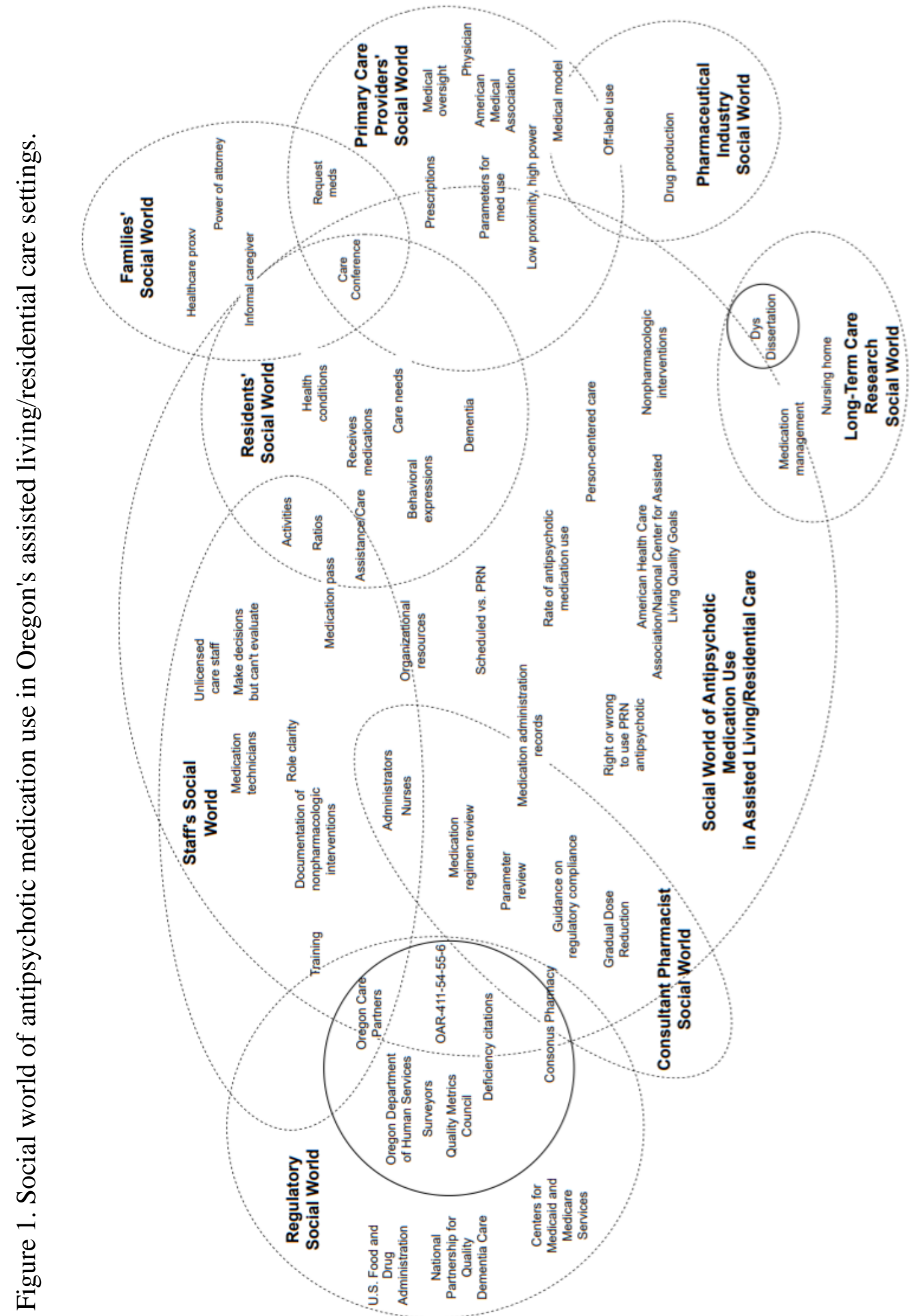


Actors within these different worlds occupy different positions within and external to the $\mathrm{AL} / \mathrm{RC}$ setting. These positions are associated with different levels of power and agency as it relates to access to antipsychotic medications (through prescriptions), parameters for use (through medication orders), and interpretations of scenarios that might result in the use of an antipsychotic medication (eg, residents' persistent agitation).

Reflecting on APU described across this dissertation, the regulatory elements associated with antipsychotics use in AL/RC settings in Oregon are reflected in administrative rules, which govern licensing of AL/RC settings. These regulations intervene on the organizational level, the AL/RC setting. These rules specify actions that staff must take regarding psychotropic medication use generally (encompassing antipsychotic medications in addition to other classes) as well as documentation requirements and a stipulation that prioritizes nonpharmacologic attempts at intervention when responding to residents' behavioral expressions or working with PRN psychotropic medication orders.

Oregon Administrative Rules intervene on staff actions, understandably because these rules govern what happens within the AL/RC setting. However, as demonstrated in this dissertation, there are factors upstream of direct care staff passing medications directly related to the use of antipsychotic medications. These study findings indicate an imbalance between power and proximity to the situation of medication situation, where those with the power to facilitate access to antipsychotic medications (ie, prescribers) are 
not the ones penalized through fines and citations for administrations that occur by those with less agency in the situation (ie, unlicensed caregivers). Creative solutions that reflect the practical reality of providing care in $\mathrm{AL} / \mathrm{RC}$ settings are sorely needed, given the extant literature regarding the substantial risks associated with the use of antipsychotic medications in older adults living with dementia ${ }^{16,22,232,281,327}$ the ethics of care in assisted living, ${ }^{298,328}$ and implications for $\mathrm{AL} / \mathrm{RC}$ resident quality of life..$^{68,230,236,237,329-332}$

\subsection{Strengths}

One of the main strengths of this dissertation study is that it embraces the complexity and contextual nature of antipsychotic medication administration and decision making in AL/RC settings. This study used three sources of data to inform the current narrative around APU in Oregon's AL/RC populations: administrative records from Oregon DHS/APD, secondary data from a state-wide survey of AL/RC settings, and primary data collected using qualitative methods and incorporating multiple perspectives. Most research of APU in older adults living with dementia is conducted in NH populations. To inform policy and practice improvements, having population and context-specific evidence is critical. This study contextualizes the situation of APU in AL/RC settings within Oregon's licensing and practice context.

Another strength of this study was the formation of a stakeholder advisory board. Especially during study design, AL/RC providers, business owners, clinical practitioners, and those with experience working and making a career out of AL/RC practice provided guidance, recommendations, and feedback. Over the course of the study period, I was in touch with stakeholders over email, offering periodic updates and opportunities to 
provide written and verbal reactions to my initial findings. These Oregon-based practitioners provided invaluable insights and reactions that helped this study take shape.

Another strength of this dissertation study is the opportunity to contribute to a small but growing literature base that centers on the minutiae of $\mathrm{AL} / \mathrm{RC}$ populations, policies, and practices. Assisted living researchers are placed in a paradox: advocating that AL/RC settings and $\mathrm{NH}$ settings are not the same, yet heavily relying on $\mathrm{NH}$ literature to frame and justify research questions and study design. In this dissertation, I lean a robust array of studies taking place in NH contexts, while a main argument of this study is that the $\mathrm{AL} / \mathrm{RC}$ context is specific and unique, particularly in Oregon. Given the growing share of $\mathrm{AL} / \mathrm{RC}$ settings as a long-term care option, ${ }^{54,119}$ it is increasingly crucial to collect data and design studies that account for AL/RC populations and settings.

Further, this study adds nuance to the empirical and practical discussion of APU in AL/RC settings. Much of the existing literature documents the prevalence of APU, associations with individual and organizational factors, and perceptions of using these medications in older adults, but in silo. Through situational analysis, I draw together and contextualize diverse data sources and perspectives that attempts to conceptualize the situation of APU in Oregon's AL/RC settings holistically.

\subsection{Limitations and Future Directions}

There are also several limitations of this study to consider. A significant limitation is that this study misses a central perspective related to AL/RC settings and APU: residents. Though each of the three studies addresses AL/RC residents tangentially, not one centers their voices or perspectives. This dissertation would be greatly strengthened by including 
residents' perspectives on medication management and the experiences of residents living in memory care and their relationships with staff and external care providers, similar to the work undertaken by Kemp and colleagues, ${ }^{153}$ Another limitation was the remote nature of performing this study. Lacking observational elements and fully utilizing myself as a research instrument in the field impacted my ability to fully conduct a situational analysis. Further, conducting this dissertation during COVID-19 pandemic that disproportionately affected congregate care settings has ethical implications. Though an important area of study, it is possible that pursuing primary data collection in the midst of a global pandemic introduced additional stress and burden to AL/RC providers and staff.

Though the in-depth interviews conducted in Chapter 5 portrayed rich narratives from the perspective of AL/RC staff, quantitative measures of staffing levels are missing. Having a sufficient number of staff to meet residents' needs is at the crux of upstream intervention to reduce the use of antipsychotic use among AL/RC residents living with ADRD. ${ }^{297,312,314}$ Oregon does not currently mandate minimum staffing ratios in AL/RC settings, though instituting minimum staffing standards in MC settings is under consideration in the state legislature. ${ }^{333}$ Recent $\mathrm{AL} / \mathrm{RC}$ findings suggest that direct care staffing policies are associated with positive health outcomes and health services utilization. ${ }^{334}$ A future study could build on this work to identify staffing models and practices associated with appropriate APU.

Regarding data used in this study, it is worth considering whether the measures and analytic design adequately contextualize APU. The deficiency citation data did not 
contain the level of detail I had expected regarding the scope of the deficiency. I was limited to what the surveyor chose to document in their report and what was publicly available. Future research could incorporate interviews with surveyors, facility staff, and residents to contextualize and improve understanding of quantitative and qualitative findings related to deficiency citations and conceptualizations of quality and safety in long-term care. Additionally, if feasible, an extension of this situational analysis could include a shadowing study of the survey process where a researcher follows along a survey team, observes the licensing survey, and sits on the debrief and issuing of citations to capture how regulatory expectations and practical realities are weighed against each other.

Finally, the purpose of this study was to contribute to the AL/RC-specific evidence base, specifically in Oregon. Though it is possible to glean many lessons from the studies presented in this dissertation, the state-specific nature of the work has implications for generalizability to other states or AL/RC contexts around the globe. The findings presented in this study should be considered within their context. It is promising that many of the findings across each of the studies reflect similar findings reported in other $\mathrm{NH}$ and $\mathrm{AL} / \mathrm{RC}$ literature. However, the findings here are discussed with the explicit purpose of informing the situation of APU within Oregon AL/RC contexts and should be interpreted as such.

\subsection{Conclusion: Public Health Significance}

This dissertation study focuses in depth on one particular situation: APU among older adults who happen to live in AL/RC settings. The specific situation of antipsychotic 
medication use in AL/RC settings has broader, public health implications to consider. Broadly, this study has public health implications related to demography, systems thinking, population health, and the future of our society.

The oldest-old in the United States population (85 years and older) are the fastest growing segment of the population. ${ }^{42}$ Most older adults will live at home in their communities until end-of-life. However, an increasing number of adults will need longterm services and supports or some form of paid caregiving at some point in their lives. ${ }^{50}$ Additionally, Alzheimer's disease, the sixth leading cause of death in the U.S. is increasing in prevalence. By 2050, an estimated 13 million adults over the age of 65 will be living with Alzheimer's disease. ${ }^{44}$ Alzheimer's disease and other dementias are terminal conditions, with no cure. Dementia symptoms largely include behavioral expressions, which can be a form of communication, articulation of pain or unmet needs, and range in severity. ${ }^{3,130}$ The increasing prevalence of older adults, and older adults living with ADRD suggest an increase for long-term care settings, including AL/RC.

If the population of people living with dementia and expressing behaviors continues to increase, with no current curative measures, it is past time to re-think what caring for and supporting those with these conditions means. This shift in population demography, increase in dementia prevalence, and nature of social and health service provision necessitates a systems level framing. Systems thinking reflects a public health vision that impacts everyone living in the U.S. AL/RC settings present the intersection of healthcare, housing, social services, and quality of life. 
The appropriateness of pharmaceutical or nonpharmacologic response to behavioral expressions in dementia is, in part, a sociocultural question. APU is often discussed in the realm of individual residents and their needs, but policy solutions target population level interventions. Understanding the appropriate characteristics associated with higher or lower rates of APU can provide insight into policy development and design of appropriate intervening mechanisms. A multilevel perspective offers opportunities for a multipronged approach to improving rates of potentially inappropriate medication use and overall quality of life for older adults living in AL/RC settings. Public health, specifically population health, offers a path forward to promote and work towards a more equitable world across the life course and for those with varying physician and cognitive abilities. 


\section{References}

1. Harris-Kojetin L, Sengupta M, Lendon J, Rome V, Valverde R, Caffrey C. Long-Term Care Providers and Services Users in the United States, 2015-2016. U.S. Department of Health and Human Services, Centers for Disease Control and Prevention, National Center for Health Statistics; 2019. https://www.cdc.gov/nchs/data/series/sr_03/sr03_43-508.pdf

2. Zimmerman S, Sloane PD, Reed D. Dementia Prevalence And Care In Assisted Living. Health Aff (Millwood). 2014;33(4):658-666. doi:10.1377/hlthaff.2013.1255

3. Cohen-Mansfield J, Dakheel-Ali M, Marx MS, Thein K, Regier NG. Which unmet needs contribute to behavior problems in persons with advanced dementia? Psychiatry Res. 2015;228(1):59-64. doi:10.1016/j.psychres.2015.03.043

4. Kales HC, Gitlin LN, Lyketsos CG. Assessment and management of behavioral and psychological symptoms of dementia. BMJ. 2015;350:h369. doi:10.1136/bmj.h369

5. Lanctôt KL, Amatniek J, Ancoli-Israel S, et al. Neuropsychiatric signs and symptoms of Alzheimer's disease: New treatment paradigms. Alzheimers Dement Transl Res Clin Interv. 2017;3(3):440-449. doi:10.1016/j.trci.2017.07.001

6. Lyketsos CG, Lopez O, Jones B, Fitzpatrick AL, Breitner J, DeKosky S. Prevalence of Neuropsychiatric Symptoms in Dementia and Mild Cognitive Impairment: Results From the Cardiovascular Health Study. JAMA. 2002;288(12):1475. doi:10.1001/jama.288.12.1475

7. Lyketsos CG. Neuropsychiatric symptoms (behavioral and psychological symptoms of dementia) and the development of dementia treatments. Int Psychogeriatr. 2007;19(3):409-420. doi:10.1017/S104161020700484X

8. Ismail Z, Smith EE, Geda Y, et al. Neuropsychiatric symptoms as early manifestations of emergent dementia: Provisional diagnostic criteria for mild behavioral impairment. Alzheimers Dement. 2016;12(2):195-202. doi:https://doi.org/10.1016/j.jalz.2015.05.017

9. Mead LC, Eckert JK, Zimmerman S, Schumacher JG. Sociocultural Aspects of Transitions From Assisted Living for Residents With Dementia. The Gerontologist. 2005;45(suppl_1):115-123. doi:10.1093/geront/45.suppl_1.115

10. Dobbs D, Zimmerman S, Carder P, Beeber AS, Palmertree SJ. Staff Reports of Behavioral Expressions of Persons with Dementia in 250 Assisted Living Communities. The Gerontologist. 2021;(gnab059). doi:10.1093/geront/gnab059

11. Aud MA. Residents with dementia in Assisted Living Facilities: The Role of Behavior in Discharge Decisions. J Gerontol Nurs. 2004;30(6):16-26. doi:10.3928/0098-9134-20040601-06

12. McKenzie G, Teri L, Pike K, LaFazia D, van Leynseele J. Reactions of Assisted Living Staff to Behavioral and Psychological Symptoms of Dementia. Geriatr Nur (Lond). 2012;33(2):96-104. doi:10.1016/j.gerinurse.2011.12.004

13. Fazio S, Zimmerman S, Doyle P, et al. What Is Really Needed to Provide Effective, Person-Centered Care for Behavioral Expressions of Dementia? Guidance from The Alzheimer's Association Dementia Care Provider Roundtable. J Am Med Dir Assoc. 2020;21(11):1582-1586.E1. doi:10.1016/j.jamda.2020.05.017 
14. Janzen S, Zecevic AA, Kloseck M, Orange JB. Managing Agitation Using Nonpharmacological Interventions for Seniors With Dementia. Am J Alzheimers Dis Dementias ${ }^{\circledR}$. 2013;28(5):524-532. doi:10.1177/1533317513494444

15. Scales K, Zimmerman S, Miller SJ. Evidence-Based Nonpharmacological Practices to Address Behavioral and Psychological Symptoms of Dementia. The Gerontologist. 2018;58(suppl_1):S88-S102. doi:10.1093/geront/gnx167

16. Maust DT, Langa KM, Blow FC, Kales HC. Psychotropic use and associated neuropsychiatric symptoms among patients with dementia in the USA. Int J Geriatr Psychiatry. 2017;32(2):164-174. doi:https://doi.org/10.1002/gps.4452

17. McKean A, Monasterio E. Off-Label Use of Atypical Antipsychotics. CNS Drugs. 2012;26(5):383-390. doi:10.2165/11632030-000000000-00000

18. O'Brien PL, Cummings N, Mark TL. Off-Label Prescribing of Psychotropic Medication, 2005-2013: An Examination of Potential Influences. Psychiatr Serv. 2017;68(6):549-558. doi:10.1176/appi.ps.201500482

19. Schneider LS, Dagerman KS, Insel P. Risk of Death With Atypical Antipsychotic Drug Treatment for Dementia: Meta-analysis of Randomized Placebo-Controlled Trials. JAMA. 2005;294(15):1934. doi:10.1001/jama.294.15.1934

20. Schneider LS, Dagerman K, Insel PS. Efficacy and Adverse Effects of Atypical Antipsychotics for Dementia: Meta-analysis of Randomized, Placebo-Controlled Trials. Am J Geriatr Psychiatry. 2006;14(3):191-210. doi:10.1097/01.JGP.0000200589.01396.6d

21. Kales HC, Kim HM, Zivin K, et al. Risk of Mortality Among Individual Antipsychotics in Patients With Dementia. Am J Psychiatry. 2012;169(1):71-79. doi:10.1176/appi.ajp.2011.11030347

22. Maust DT, Kim HM, Seyfried LS, et al. Antipsychotics, Other Psychotropics, and the Risk of Death in Patients With Dementia: Number Needed to Harm. JAMA Psychiatry. 2015;72(5):438. doi:10.1001/jamapsychiatry.2014.3018

23. Stock KJ, Hogan DB, Lapane K, et al. Antipsychotic Use and Hospitalization Among Older Assisted Living Residents: Does Risk Vary by Frailty Status? Am J Geriatr Psychiatry. 2017;25(7):779-790. doi:10.1016/j.jagp.2017.02.013

24. Lucas JA, Chakravarty S, Bowblis JR, et al. Antipsychotic medication use in nursing homes: a proposed measure of quality: Quality measure of APM use in NHS. Int J Geriatr Psychiatry. 2014;29(10):1049-1061. doi:10.1002/gps.4098

25. Crystal S, Jarrín OF, Rosenthal M, Hermida R, Angell B. National Partnership to Improve Dementia Care in Nursing Homes Campaign: State and Facility Strategies, Impact, and Antipsychotic Reduction Outcomes. Innov Aging. 2020;4(igaa018). doi:10.1093/geroni/igaa018

26. American Health Care Association/National Center for Assisted Living Quality Initiative. Assisted Living Goals. AHCA/NCAL. Published 2020. Accessed January 12, 2020. https://www.ahcancal.org/Quality/Quality-Initiative/Pages/AL-goals.aspx

27. Lapane KL. Reducing Off-Label Antipsychotic Use in Older Adults: Time to Look Beyond the Doors of Nursing Homes. J Am Geriatr Soc. 2018;66(6):1055-1057. doi:https://doi.org/10.1111/jgs.15262 
28. Zimmerman S, Scales K, Wiggins B, Cohen LW, Sloane PD. Addressing Antipsychotic Use in Assisted Living Residents with Dementia. J Am Geriatr Soc. 2015;63(9):1970-1971. doi:https://doi.org/10.1111/jgs.13625

29. Kerns JW, Winter JD, Winter KM, Kerns CC, Etz RS. Caregiver Perspectives About Using Antipsychotics and Other Medications for Symptoms of Dementia. The Gerontologist. 2018;58(2):e35-e45. doi:10.1093/geront/gnx042

30. Kerns JW, Winter JD, Winter KM, Boyd T, Etz RS. Primary Care Physician Perspectives about Antipsychotics and Other Medications for Symptoms of Dementia. J Am Board Fam Med. 2018;31(1):9-21. doi:10.3122/jabfm.2018.01.170230

31. Ryder PT, Joseph M, Zuckerman M, Zuckerman IH. Administrators' Perceptions of Medication Management in Assisted Living Facilities: Results from Focus Groups. Consult Pharm J Am Soc Consult Pharm. 2009;24(7):513-523.

32. Kirkham J, Sherman C, Velkers C, et al. Antipsychotic Use in Dementia: Is There a Problem and Are There Solutions? Can J Psychiatry. 2017;62(3):170-181. doi: $10.1177 / 0706743716673321$

33. Ostrom E. An agenda for the study of institutions. Public Choice. 1986;48(1). doi:10.1007/BF00239556

34. Crawford SES, Ostrom E. A Grammar of Institutions. Am Polit Sci Rev. 1995;89(3):582-600. doi:10.2307/2082975

35. Ostrom E. Institutional Rational Choice: An Assessment of the Institutional Analysis and Development Framework. In: Theories of the Policy Process. Routledge; 2007.

36. Ostrom E. Institutional Analysis and Development: Elements of the framework in historical perspective. In: Crothers C, ed. Historical Developments and Theoretical Approaches in Sociology. Vol 2. EOLSS Publications; 2010:261-288.

37. McGinnis MD. An Introduction to IAD and the Language of the Ostrom Workshop: A Simple Guide to a Complex Framework. Policy Stud J. 2011;39(1):169-183. doi:10.1111/j.1541-0072.2010.00401.x

38. Oregon Quality Measurement Council. Quality Measurement Program Report for Oregon Community-Based Care. Oregon Department of Human Services, Office of Aging and People with Disabilities; 2020:71. Accessed October 10, 2021. https://www.oregon.gov/dhs/PROVIDERSPARTNERS/LICENSING/CBC/QMCReports/Quality-Measurement-Report2020.pdf

39. Oregon State Legislature. Relating to Psychotropic Medication.; 2017. https://olis.leg.state.or.us/liz/2017R1/Downloads/MeasureDocument/HB3262.

40. Office of the Governor State of Oregon. Declaration of Emergency Due to Coronavirus (COVID-19) Outbreak in Oregon. Vol 20-03.; 2020. Accessed October 11, 2021. https://www.oregon.gov/gov/Documents/executive_orders/eo_20-03.pdf

41. Kaiser Family Foundation. State COVID-19 Data and Policy Actions. KFF. Published October 5, 2021. Accessed October 11, 2021. https://www.kff.org/coronavirus-covid-19/issue-brief/state-covid-19-data-andpolicy-actions/ 
42. Vespa J, Armstrong DM, Medina L. Demographic Turning Points for the United States: Population Projections for 2020 to 2060. U.S. Census Bureau; 2020. Accessed October 2, 2021. https://www.census.gov/library/publications/2020/demo/p25-1144.html

43. Kramarow EA, Tejada-Vera B. Dementia Mortality in the United States, 2000-2017. Natl Vital Stat Rep. 2019;68(2). https://www.cdc.gov/nchs/data/nvsr/nvsr68/nvsr68_02-508.pdf

44. 2021 Alzheimer's disease facts and figures. Alzheimers Dement J Alzheimers Assoc. 2021;17(3):327-406. doi:10.1002/alz.12328

45. Hugo J, Ganguli M. Dementia and Cognitive Impairment: Epidemiology, Diagnosis, and Treatment. Clin Geriatr Med. 2014;30(3):421-442. doi:10.1016/j.cger.2014.04.001

46. Borson S, Frank L, Bayley PJ, et al. Improving dementia care: The role of screening and detection of cognitive impairment. Alzheimers Dement. 2013;9(2):151-159. doi:10.1016/j.jalz.2012.08.008

47. Kasper JD, Freedman VA, Spillman BC, Wolff JL. The Disproportionate Impact Of Dementia On Family And Unpaid Caregiving To Older Adults. Health Aff (Millwood). 2015;34(10):1642-1649. doi:10.1377/hlthaff.2015.0536

48. Jutkowitz E, Kane RL, Gaugler JE, MacLehose RF, Dowd B, Kuntz KM. Societal and family lifetime cost of dementia: Implications for policy. J Am Geriatr Soc. 2017;65(10):2169-2175. doi:10.1111/jgs.15043

49. Chang CH, Ming Y, Chang TH, Yen YY, Lan SJ. The Needs and Utilization of LongTerm Care Service Resources by Dementia Family Caregivers and the Affecting Factors. Int J Environ Res Public Health. 2020;17(16):6009. doi:10.3390/ijerph17166009

50. Johnson RW. What Is the Lifetime Risk of Needing and Receiving Long-Term Services and Supports? Department of Health and Human Services Office of the Assistant Secretary for Planning and Evaluation; 2019. Accessed October 2, 2021. https://aspe.hhs.gov/reports/what-lifetime-risk-needing-receiving-long-termservices-supports-0

51. Thomas KS, Applebaum R. Long-term Services and Supports (LTSS): A Growing Challenge for an Aging America. Public Policy Aging Rep. 2015;25(2):56-62. doi:10.1093/ppar/prv003

52. Stevenson DG, Grabowski DC. Sizing Up The Market For Assisted Living. Health Aff (Millwood). 2010;29(1):35-43. doi:10.1377/hlthaff.2009.0527

53. Grabowski DC, Stevenson DG, Cornell PY. Assisted Living Expansion and the Market for Nursing Home Care. Health Serv Res. 2012;47(6):2296-2315. doi:10.1111/j.1475-6773.2012.01425.x

54. Cornell PY, Zhang W, Thomas KS. Changes in Long-Term Care Markets: Assisted Living Supply and the Prevalence of Low-Care Residents in Nursing Homes. J Am Med Dir Assoc. 2020;21(8):1161-1165.e4. doi:10.1016/j.jamda.2020.01.006

55. Magsi H, Malloy T. Underrecognition of Cognitive Impairment in Assisted Living Facilities. J Am Geriatr Soc. 2005;53(2):295-298. doi:https://doi.org/10.1111/j.15325415.2005.53117.x 
56. Lang L, Clifford A, Wei L, et al. Prevalence and determinants of undetected dementia in the community: a systematic literature review and a meta-analysis. BMJ Open. 2017;7(2):e011146. doi:10.1136/bmjopen-2016-011146

57. Cipriani G, Lucetti C, Danti S, Nuti A. Sleep disturbances and dementia. Psychogeriatrics. 2015;15(1):65-74. doi:10.1111/psyg.12069

58. Cipriani G, Lucetti C, Carlesi C, Danti S, Nuti A. Sundown syndrome and dementia. Eur Geriatr Med. 2015;6(4):375-380. doi:10.1016/j.eurger.2015.03.006

59. Lyketsos CG, Carrillo MC, Ryan JM, et al. Neuropsychiatric symptoms in Alzheimer's disease. Alzheimers Dement. 2011;7(5):532-539. doi:https://doi.org/10.1016/j.jalz.2011.05.2410

60. Steinberg M, Shao H, Zandi P, et al. Point and 5-year period prevalence of neuropsychiatric symptoms in dementia: the Cache County Study. Int J Geriatr Psychiatry. 2008;23(2):170-177. doi:https://doi.org/10.1002/gps.1858

61. Gitlin LN, Marx KA, Stanley IH, Hansen BR, Van Haitsma KS. Assessing neuropsychiatric symptoms in people with dementia: a systematic review of measures. Int Psychogeriatr. 2014;26(11):1805-1848. doi:10.1017/S1041610214001537

62. Soril LJJ, Leggett LE, Lorenzetti DL, et al. Effective Use of the Built Environment to Manage Behavioural and Psychological Symptoms of Dementia: A Systematic Review. PLOS ONE. 2014;9(12):e115425. doi:10.1371/journal.pone.0115425

63. Kolanowski A, Boltz M, Galik E, et al. Determinants of behavioral and psychological symptoms of dementia: A scoping review of the evidence. Nurs Outlook. 2017;65(5):515-529. doi:10.1016/j.outlook.2017.06.006

64. Gaugler JE, Kane RL, Kane RA, Newcomer R. Unmet care needs and key outcomes in dementia. J Am Geriatr Soc. 2005;53(12):2098-2105. doi:10.1111/j.15325415.2005.00495.x

65. Mitty E, Flores S. Assisted living nursing practice: the language of dementia: theories and interventions. Geriatr Nurs N Y N. 2007;28(5):283-288. doi:10.1016/j.gerinurse.2007.08.009

66. Gruber-Baldini AL, Boustani M, Sloane PD, Zimmerman S. Behavioral symptoms in residential care/assisted living facilities: prevalence, risk factors, and medication management. J Am Geriatr Soc. 2004;52(10):1610-1617. doi:10.1111/j.15325415.2004.52451.x

67. Angeles RC, Berge LI, Gedde MH, et al. Which factors increase informal care hours and societal costs among caregivers of people with dementia? A systematic review of Resource Utilization in Dementia (RUD). Health Econ Rev. 2021;11(1):37. doi:10.1186/s13561-021-00333-z

68. Karttunen K, Karppi P, Hiltunen A, et al. Neuropsychiatric symptoms and Quality of Life in patients with very mild and mild Alzheimer's disease. Int J Geriatr Psychiatry. 2011;26(5):473-482. doi:https://doi.org/10.1002/gps.2550

69. Branger C, Enright J, O'Connell ME, Morgan DG. Variance in caregiver burden predicted by patient behaviors versus neuropsychological profile. Appl Neuropsychol Adult. 2018;25(5):441-447. doi:10.1080/23279095.2017.1323754 
70. Zwijsen SA, Kabboord A, Eefsting JA, et al. Nurses in distress? An explorative study into the relation between distress and individual neuropsychiatric symptoms of people with dementia in nursing homes. Int J Geriatr Psychiatry. 2014;29(4):384391. doi:10.1002/gps.4014

71. Huang D, Kleiman S, Wasserman J. 207 National Trends in Geriatric Emergency Department Visits: Demographics, Morbidities, and Hospital Admission Factors, 2011-2015. Ann Emerg Med. 2018;72(4):S83. doi:10.1016/j.annemergmed.2018.08.212

72. Fazio S, Pace D, Flinner J, Kallmyer B. The Fundamentals of Person-Centered Care for Individuals With Dementia. The Gerontologist. 2018;58(suppl_1):S10-S19. doi:10.1093/geront/gnx122

73. Kim SK, Park M. Effectiveness of person-centered care on people with dementia: a systematic review and meta-analysis. Clin Interv Aging. 2017;12:381-397. doi:10.2147/CIA.S117637

74. Cohen-Mansfield J, Thein K, Marx MS, Dakheel-Ali M, Freedman L. Efficacy of nonpharmacologic interventions for agitation in advanced dementia: a randomized, placebo-controlled trial. J Clin Psychiatry. 2012;73(9):1255-1261. doi:10.4088/JCP.12m07918

75. Barbosa A, Sousa L, Nolan M, Figueiredo D. Effects of Person-Centered Care Approaches to Dementia Care on Staff: A Systematic Review. Am J Alzheimers Dis Other Demen. 2015;30(8):713-722. doi:10.1177/1533317513520213

76. Chenoweth L, Stein-Parbury J, Lapkin S, Wang A, Liu Z, Williams A. Effects of person-centered care at the organisational-level for people with dementia. A systematic review. PLOS ONE. 2019;14(2):e0212686. doi:10.1371/journal.pone.0212686

77. Reus VI, Fochtmann LJ, Eyler AE, et al. The American Psychiatric Association Practice Guideline on the Use of Antipsychotics to Treat Agitation or Psychosis in Patients With Dementia. Am J Psychiatry. 2016;173(5):543-546. doi:10.1176/appi.ajp.2015.173501

78. Vaismoradi M, Vizcaya Moreno F, Sletvold H, Jordan S. PRN Medicines Management for Psychotropic Medicines in Long-Term Care Settings: A Systematic Review. Pharmacy. 2019;7(4):157. doi:10.3390/pharmacy7040157

79. Caraci F, Enna SJ, Zohar J, et al. A new nomenclature for classifying psychotropic drugs. Br J Clin Pharmacol. 2017;83(8):1614-1616. doi:10.1111/bcp.13302

80. Seifert R, Schirmer B. A simple mechanistic terminology of psychoactive drugs: a proposal. Naunyn Schmiedebergs Arch Pharmacol. 2020;393(8):1331-1339. doi:10.1007/s00210-020-01918-x

81. Panel B the 2019 AGSBCUE. American Geriatrics Society 2019 Updated AGS Beers Criteria ${ }^{\circledR}$ for Potentially Inappropriate Medication Use in Older Adults. $J$ Am Geriatr Soc. 2019;67(4):674-694. doi:10.1111/jgs.15767

82. Ma H, Huang Y, Cong Z, et al. The efficacy and safety of atypical antipsychotics for the treatment of dementia: a meta-analysis of randomized placebo-controlled trials. $J$ Alzheimers Dis JAD. 2014;42(3):915-937. doi:10.3233/JAD-140579 
83. Salzman C, Jeste DV, Meyer RE, et al. Elderly patients with dementia-related symptoms of severe agitation and aggression: consensus statement on treatment options, clinical trials methodology, and policy. J Clin Psychiatry. 2008;69(6):889898. doi:10.4088/jcp.v69n0602

84. Sturm AS, Trinkley KE, Porter K, Nahata MC. Efficacy and safety of atypical antipsychotics for behavioral symptoms of dementia among patients residing in long-term care. Int J Clin Pharm. 2018;40(1):135-142. doi:10.1007/s11096-017$0555-y$

85. Yunusa I, Alsumali A, Garba AE, Regestein QR, Eguale T. Assessment of Reported Comparative Effectiveness and Safety of Atypical Antipsychotics in the Treatment of Behavioral and Psychological Symptoms of Dementia: A Network Meta-analysis. JAMA Netw Open. 2019;2(3):e190828. doi:10.1001/jamanetworkopen.2019.0828

86. Beeber AS, Zimmerman S, Wretman CJ, Palmertree S, Patel K, Sloane PD. Potential Side Effects and Adverse Events of Antipsychotic Use for Residents With Dementia in Assisted Living: Implications for Prescribers, Staff, and Families. J Appl Gerontol. Published online June 23, 2021:07334648211023678. doi:10.1177/07334648211023678

87. Huang AR, Mallet L, Rochefort CM, Eguale T, Buckeridge DL, Tamblyn R. Medication-related falls in the elderly: causative factors and preventive strategies. Drugs Aging. 2012;29(5):359-376. doi:10.2165/11599460-000000000-00000

88. Maust DT, Kim HM, Chiang C, Langa KM, Kales HC. Predicting Risk of Potentially Preventable Hospitalization in Older Adults with Dementia. J Am Geriatr Soc. 2019;67(10):2077-2084. doi:10.1111/jgs.16030

89. Schneeweiss S, Setoguchi S, Brookhart A, Dormuth C, Wang PS. Risk of death associated with the use of conventional versus atypical antipsychotic drugs among elderly patients. Can Med Assoc J. 2007;176(5):627-632. doi:10.1503/cmaj.061250

90. Huybrechts KF, Gerhard T, Crystal S, et al. Differential risk of death in older residents in nursing homes prescribed specific antipsychotic drugs: population based cohort study. BMJ. 2012;344(feb23 2):e977-e977. doi:10.1136/bmj.e977

91. Wang PS, Schneeweiss S, Avorn J, et al. Risk of Death in Elderly Users of Conventional vs. Atypical Antipsychotic Medications. N Engl J Med. 2005;353(22):2335-2341. doi:10.1056/NEJMoa052827

92. Alzheimer's Association, Centers for Disease Control and Prevention. Healthy Brain Initiative, State and Local Public Health Partnerships to Address Dementia: The 2018-2023 Road Map. Alzheimer's Association; 2018. https://www.alz.org/media/Documents/healthy-brain-initiative-road-map-20182023.pdf

93. United States Food and Drug Administration. Public Health Advisory: Deaths with Antipsychotics in Elderly Patients with Behavioral Disturbances. U.S. Food and Drug Administration. Published April 11, 2005. Accessed February 7, 2020. https://wayback.archiveit.org/7993/20170113112252/http://www.fda.gov/Drugs/DrugSafety/PostmarketDru gSafetyInformationforPatientsandProviders/ucm053171.htm 
94. Kuehn BM. FDA warns antipsychotic drugs may be risky for elderly. JAMA. 2005;293(20):2462. doi:10.1001/jama.293.20.2462

95. Dorsey ER, Rabbani A, Gallagher SA, Conti RM, Alexander GC. Impact of FDA Black Box Advisory on Antipsychotic Medication Use. Arch Intern Med. 2010;170(1):96-103. doi:10.1001/archinternmed.2009.456

96. Austrom MG, Boustani M, LaMantia MA. Ongoing Medical Management to Maximize Health and Well-being for Persons Living With Dementia. The Gerontologist. 2018;58(suppl_1):S48-S57. doi:10.1093/geront/gnx147

97. Rattinger GB, Burcu M, Dutcher SK, et al. Pharmacotherapeutic Management of Dementia Across Settings of Care. J Am Geriatr Soc. 2013;61(5):723-733. doi: $10.1111 /$ jgs. 12210

98. McDermott CL, Gruenewald DA. Pharmacologic Management of Agitation in Patients with Dementia. Curr Geriatr Rep. 2019;8(1):1-11. doi:10.1007/s13670-0190269-1

99. Gill D, Almutairi S, Donyai P. "The Lesser of Two Evils" Versus "Medicines not Smarties": Constructing Antipsychotics in Dementia. The Gerontologist. 2019;59(3):570-579. doi:10.1093/geront/gnx178

100. Chen J, Bell S, Brain C. Healthcare providers' perceptions of the efficacy of antipsychotics in dementia-related psychosis. Alzheimers Dement. 2020;16(S8):e047139. doi:https://doi.org/10.1002/alz.047139

101. Langford AV, Ngo GT, Chen TF, Roberts C, Schneider CR. Nurses', Pharmacists' and Family Physicians' Perceptions of Psychotropic Medication Monitoring in Australian Long-Term Care Facilities: A Qualitative Framework Analysis. Drugs Aging. 2021;38(2):169-179. doi:10.1007/s40266-020-00825-3

102. Lucas JA, Bowblis JR. CMS Strategies To Reduce Antipsychotic Drug Use In Nursing Home Patients With Dementia Show Some Progress. Health Aff (Millwood). 2017;36(7):1299-1308. doi:10.1377/hlthaff.2016.1439

103. Maust DT, Kim HM, Chiang C, Kales HC. Association of the Centers for Medicare \& Medicaid Services' National Partnership to Improve Dementia Care With the Use of Antipsychotics and Other Psychotropics in Long-term Care in the United States From 2009 to 2014. JAMA Intern Med. 2018;178(5):640-647. doi:10.1001/jamainternmed.2018.0379

104. Centers for Medicare and Medicaid Services. National Partnership to Improve Dementia Care in Nursing Homes: Antipsychotic Medication Use Data Report. Centers for Medicare and Medicaid Services; 2019. Accessed October 12, 2021. https://www.cms.gov/Medicare/Provider-Enrollment-andCertification/SurveyCertificationGenInfo/Downloads/Antipsychotic-MedicationUse-Data-Report.pdf

105. Cepoiu-Martin M, Tam-Tham H, Patten S, Maxwell CJ, Hogan DB. Predictors of long-term care placement in persons with dementia: a systematic review and metaanalysis. Int J Geriatr Psychiatry. 2016;31(11):1151-1171. doi:10.1002/gps.4449

106. Martin A, Tyrrell S, Honey E, Rodgers J, Ingham B. What Promotes Transfer of Staff Training Related to Challenging Behavior Displayed by Adults With 
Intellectual Disabilities? J Policy Pract Intellect Disabil. n/a(n/a).

doi:10.1111/jppi.12393

107. Berry B, Apesoa-Varano EC. Medication takeovers: Covert druggings and behavioral control in Alzheimer's. Soc Sci Med. 2017;188:51-59. doi:10.1016/j.socscimed.2017.07.003

108. Ralph SJ, Espinet AJ. Use of antipsychotics and benzodiazepines for dementia: Time for action? What will be required before global de-prescribing? Dementia. 2019;18(6):2322-2339. doi:10.1177/1471301217746769

109. Ferreira P, Ferreira AR, Barreto B, Fernandes L. Is there a link between the use of benzodiazepines and related drugs and dementia? A systematic review of reviews. Eur Geriatr Med. Published online August 17, 2021. doi:10.1007/s41999-02100553-w

110. Kales HC, Gitlin LN, Lyketsos CG. When Less is More, but Still Not Enough: Why Focusing on Limiting Antipsychotics in People With Dementia Is the Wrong Policy Imperative. J Am Med Dir Assoc. 2019;20(9):1074-1079. doi:10.1016/j.jamda.2019.05.022

111. Carder PC, O’Keeffe J, O'Keeffee C. Compendium of Residential Care and Assisted Living Regulations and Policy: 2015 Edition Executive Summary. RTI International; 2015. https://aspe.hhs.gov/execsum/compendium-residential-care-and-assistedliving-regulations-and-policy-2015-edition-executive-summary

112. Carder PC. State Regulatory Approaches for Dementia Care in Residential Care and Assisted Living. The Gerontologist. 2017;57(4):776-786. doi:10.1093/geront/gnw197

113. National Center for Assisted Living. 2019 Assisted Living State Regulatory Review. American Health Care Association/National Center for Assisted Living; 2019. Accessed October 12, 2021. https://www.ahcancal.org/AssistedLiving/Policy/Documents/2019_reg_review.pdf

114. Trinkoff AM, Yoon JM, Storr CL, Lerner NB, Yang BK, Han K. Comparing residential long-term care regulations between nursing homes and assisted living facilities. Nurs Outlook. 2020;68(1):114-122. doi:10.1016/j.outlook.2019.06.015

115. Regnier V, Hamilton J, Yatabe S. Assisted Living for the Aged and Frail: Innovations in Design, Management, and Financing. Columbia University Press; 1995.

116. Brown Wilson K. Historical Evolution of Assisted Living in the United States, 1979 to the Present. The Gerontologist. 2007;47(suppl_1):8-22. doi:10.1093/geront/47.Supplement_1.8

117. U.S. Census Bureau QuickFacts: Oregon. Accessed October 12, 2021. https://www.census.gov/quickfacts/fact/table/OR/AGE775219\#AGE775218

118. Vaidya KL. Oregon's Demographic Trends. Office of Economic Analysis, Department of Administrative Services; 2019. Accessed October 12, 2021. https://www.oregon.gov/das/OEA/Documents/OR_pop_trend2019.pdf

119. Cornell PY, Zhang W, Smith L, Fashaw S, Thomas KS. Developments in the Market for Assisted Living: Residential Care Availability in 2017. J Am Med Dir Assoc. 2020;21(11):1718-1723. doi:10.1016/j.jamda.2020.08.011 
120. Carder P, Kohon J, Limburg A, West M, Zimam A, Margaret B. Neal. Oregon Community-Based Care: Resident and Community Characteristics Report. Institute on Aging, Portland State University; 2014. Accessed October 12, 2021. https://www.pdx.edu/institute-on-aging/sites/g/files/znldhr3046/files/202007/2014_CBC_AnnualReport_AL-RC-MC.pdf

121. Carder PC, Elliott S, Tunalilar O, Dys S. 2019 Community-Based Care: Resident and Community Characteristics Report on Assisted Living, Residential Care, Memory Care. Institute on Aging, Portland State University; 2019.

122. Oregon Department of Human Services, Aging and People with Disabilities. Residential Care and Assisted Living Facilities.; 2020. https://www.dhs.state.or.us/policy/spd/rules/411_054.pd

123. Oregon Department of Human Services, Aging and People with Disabilities. Memory Care Communities.; 2020. https://www.dhs.state.or.us/policy/spd/rules/411_057.pdf

124. Mendez-Luck C, Luck J, Larson A, Dyer G. The State of Nursing Facilities in Oregon, 2017. Oregon State University; 2018.

https://www.oregon.gov/DHS/SENIORS-DISABILITIES/Documents/2017-OregonNursing-Facilities-Report.pdf

125. Hughes CM, Lapane KL, Mor V. Influence of facility characteristics on use of antipsychotic medications in nursing homes. Med Care. 2000;38(12):1164-1173. doi:10.1097/00005650-200012000-00003

126. Kamble P, Chen H, Sherer J, Aparasu RR. Antipsychotic drug use among elderly nursing home residents in the United States. Am J Geriatr Pharmacother. 2008;6(4):187-197. doi:10.1016/j.amjopharm.2008.10.002

127. Stevenson DG, Decker SL, Dwyer LL, et al. Antipsychotic and benzodiazepine use among nursing home residents: findings from the 2004 National Nursing Home Survey. Am J Geriatr Psychiatry Off J Am Assoc Geriatr Psychiatry. 2010;18(12):1078-1092. doi:10.1097/JGP.0b013e3181d6c0c6

128. Cioltan H, Alshehri S, Howe C, et al. Variation in use of antipsychotic medications in nursing homes in the United States: A systematic review. BMC Geriatr. 2017;17(1):1-12. doi:10.1186/s12877-017-0428-1

129. Sloane PD, Gruber-Baldini AL, Zimmerman S, et al. Medication Undertreatment in Assisted Living Settings. Arch Intern Med. 2004;164(18):2031-2037. doi:10.1001/archinte.164.18.2031

130. Brecher DB, West TL. Underrecognition and Undertreatment of Pain and Behavioral Symptoms in End-Stage Dementia. Am J Hosp Palliat Med. 2016;33(3):276-280. doi:10.1177/1049909114559069

131. Reese TR, Thiel DJ, Cocker K. Behavioral Disorders in Dementia: Appropriate Nondrug Interventions and Antipsychotic Use. Am Fam Physician. 2016;94(4):276282.

132. Stock KJ, Amuah JE, Lapane KL, Hogan DB, Maxwell CJ. Prevalence of, and Resident and Facility Characteristics Associated With Antipsychotic Use in Assisted Living vs. Long-Term Care Facilities: A Cross-Sectional Analysis from Alberta, Canada. Drugs Aging. 2017;34(1):39-53. doi:10.1007/s40266-016-0411-0 
133. Thomas KS, Wretman CJ, Sloane PD, et al. To What Extent Do Local Nursing Home Prescribing Patterns Relate to Psychotropic Prescribing in Assisted Living? J Am Med Dir Assoc. 2021;22(9):1813-1818.e3. doi:10.1016/j.jamda.2020.11.037

134. Dys S, Tunalilar O, Carder P. Cognition-Enhancing, Antipsychotic, and Opioid Medication Use Among Assisted Living and Residential Care Residents in Oregon. $J$ Am Med Dir Assoc. Published online January 28, 2021. doi:10.1016/j.jamda.2020.12.030

135. Oregon Health Care Association. CBC Survey Data. Oregon Health Care Association. Published 2019. Accessed October 15, 2021. https://www.ohca.com/members/research-and-data/cbc-survey-data/

136. Oregon State Legislature. Relating to Long-Term Care.; 2017. https://olis.leg.state.or.us/liz/2017R1/Downloads/MeasureDocument/HB3359.

137. Oregon Quality Metrics Council. Quality Metrics Council Meeting Minutes. Published online April 25, 2019. Accessed October 15, 2021. https://www.oregon.gov/DHS/PROVIDERSPARTNERS/LICENSING/CBC/QMPMtgDocs/4-2519\%20QMC\%20Meeting\%20Minutes\%205-15-19.pdf

138. Oregon Department of Human Services, Aging and People with Disabilities. Quality Measurement Program Report for Oregon Community-Based Care Facilities. Published online 2020:71.

139. Kemp CL, Luo S, Ball MM. "Meds are a Real Tricky Area": Examining Medication Management and Regulation in Assisted Living. J Appl Gerontol. 2012;31(1):126149. doi: $10.1177 / 0733464810381986$

140. Carder PC, O'Keeffe J. State Regulation of Medication Administration by Unlicensed Assistive Personnel in Residential Care and Adult Day Services Settings. Res Gerontol Nurs. 2016;9(5):209-222. doi:10.3928/19404921-20160404-03

141. Carder PC. "Learning About Your Residents": How Assisted Living Residence Medication Aides Decide to Administer Pro Re Nata Medications to Persons With Dementia. The Gerontologist. 2012;52(1):46-55. doi:10.1093/geront/gnr099

142. Sikma SK, Young HM, Reinhard SC, Munroe DJ, Cartwright J, McKenzie G. Medication management roles in assisted living. J Gerontol Nurs. 2014;40(6):42-53. doi:10.3928/00989134-20140211-02

143. Young HM, Sikma SK, Reinhard S, McCormick WC, Cartwright JC. Strategies to Promote Safe Medication Administration in Assisted Living Settings. Res Gerontol Nurs. 2013;6(3):161-170. doi:10.3928/19404921-20130122-01

144. Coulson E, Blaszczyk AT. Assisted Living Facilities: The Next Frontier for Consultant Pharmacists. Consult Pharm. 2016;31(2):107-111. doi:10.4140/TCP.n.2016.107

145. Ball MM, Lepore ML, Perkins MM, Hollingsworth C, Sweatman M. "They Are the Reason I Come to Work": The Meaning of Resident-Staff Relationships in Assisted Living. J Aging Stud. 2009;23(1):37-47. doi:10.1016/j.jaging.2007.09.006

146. Bowman SE, Rogers WA. Understanding Decision Making Among Direct Care Workers in Assisted Living. J Cogn Eng Decis Mak. 2016;10(4):369-390. doi:10.1177/1555343416656952 
147. Oregon Board of Nursing. Standards for Community-Based Care Registered Nurse Delegation Process.; 2004.

https://secure.sos.state.or.us/oard/displayDivisionRules.action?selectedDivision=393 1

148. Torres-Pomales A. Responding To Behavioral Expressions Of Residents Living With Dementia In Assisted Living. Published online May 10, 2019. Accessed February 15, 2020. https://scholarworks.gsu.edu/gerontology_theses/45

149. Crawford SES, Ostrom E. A grammar of institutions. Am Polit Sci Rev. 1995;89(3). Accessed October 13, 2021. https://go-galecom.proxy.lib.pdx.edu/ps/i.do?p=PROF\&u=s1185784\&id=GALE\%7CA17510084\& $\mathrm{v}=2.1 \& \mathrm{it}=\mathrm{r}$

150. Kiser LL, Ostrom E. The Three Worlds of Action: A Metatheoretical Synthesis of Institutional Approaches. In: Ostrom E, ed. Strategies of Political Inquiry. Sage; 1982:179-222.

151. Ostrom E. Background on the Institutional Analysis and Development Framework. Policy Stud J. 2011;39(1):7-27. doi:10.1111/j.1541-0072.2010.00394.x

152. Kemp CL, Ball MM, Perkins MM. Convoys of care: Theorizing intersections of formal and informal care. J Aging Stud. 2013;27(1):15-29. doi:10.1016/j.jaging.2012.10.002

153. Kemp CL, Ball MM, Morgan JC, Doyle PJ, Burgess EO, Perkins MM. Maneuvering Together, Apart, and at Odds: Residents' Care Convoys in Assisted Living. $J$ Gerontol Ser B. 2018;73(4):e13-e23. doi:10.1093/geronb/gbx184

154. Kemp CL, Ball MM, Perkins MM. Individualization and the Health Care Mosaic in Assisted Living. The Gerontologist. 2019;59(4):644-654. doi:10.1093/geront/gny065

155. Clement F. Analysing decentralised natural resource governance: proposition for a "politicised" institutional analysis and development framework. Policy Sci. 2010;43(2):129-156. doi:10.1007/s11077-009-9100-8

156. Carter DP, Weible CM, Siddiki SN, Basurto X. Integrating core concepts from the institutional analysis and development framework for the systematic analysis of policy designs: An illustration from the US National Organic Program regulation. $J$ Theor Polit. 2016;28(1):159-185. doi:10.1177/0951629815603494

157. Barton DN, Benavides K, Chacon-Cascante A, et al. Payments for Ecosystem Services as a Policy Mix: Demonstrating the institutional analysis and development framework on conservation policy instruments. Environ Policy Gov. 2017;27(5):404-421. doi:10.1002/eet.1769

158. Bryant A. Grounded Theory and Pragmatism: The Curious Case of Anselm Strauss. Forum Qual Sozialforschung Forum Qual Soc Res. 2009;10(3). doi:10.17169/fqs10.3.1358

159. Morgan DL. Pragmatism as a Paradigm for Social Research. Qual Inq. 2014;20(8):1045-1053. doi:10.1177/1077800413513733

160. Charmaz K. Constructing Grounded Theory. SAGE; 2014.

161. Kelly LM, Cordeiro M. Three principles of pragmatism for research on organizational processes. Methodol Innov. 2020;13(2):2059799120937242. doi: $10.1177 / 2059799120937242$ 
162. Morgan DL. Pragmatism as a Basis for Grounded Theory. :11.

163. Clarke AE. Situational Analyses: Grounded Theory Mapping After the Postmodern Turn. Symb Interact. 2003;26(4):553-576. doi:10.1525/si.2003.26.4.553

164. Strauss A, Corbin JM. Grounded Theory in Practice. SAGE; 1997.

165. Strauss A, Corbin J. Basics of Qualitative Research: Techniques and Procedures for Developing Grounded Theory. 2nd ed. SAGE Publications, Inc.; 1998.

166. Morse JM, Bowers BJ, Charmaz K, Corbin J, Clarke AE, Stern PN. Developing Grounded Theory: The Second Generation. Routledge; 2016.

167. Strauss A. A Social World Perspective. Stud Symb Interact. 1978;1:119-128.

168. Strauss A. Social worlds and legitimation processes. Stud Symb Interact. 1982;4:171-190.

169. Strauss A. Interorganizational Negotiation. Urban Life. 1982;11(3):350-367. doi:10.1177/089124168201100306

170. Strauss A. Social worlds and their segmentation processes. Stud Symb Interact. 1984;5:123-139.

171. Carder PC. The social world of assisted living. J Aging Stud. 2002;16(1):1-18. doi:10.1016/S0890-4065(01)00031-7

172. Carder PC. Managing medication management in assisted living: A situational analysis. J Ethnogr Qual Res. 2008;3:1-12.

173. Clarke AE. Situational Analysis. In: The Blackwell Encyclopedia of Sociology. American Cancer Society; 2016:1-2. doi:10.1002/9781405165518.wbeos0777

174. Clarke AE, Friese C, Washburn R, eds. Situational Analysis in Practice: Mapping Research with Grounded Theory. Routledge; 2016. doi:10.4324/9781315420134

175. Clarke AE, Friese C, Washburn RS. Situational Analysis: Grounded Theory After the Interpretive Turn. SAGE Publications; 2017.

176. Clarke AE, Charmaz K. Grounded Theory and Situational Analysis. In: Atkinson P, Delamont S, Cernat A, Sakshaug JW, Williams RiA, eds. SAGE Research Methods Foundations. Qualitative Analysis. SAGE Publications Ltd; 2019. doi: $10.4135 / 9781526421036825838$

177. Clarke AE. From Grounded Theory to Situational Analysis: What's New? Why? How? In: Morse JM, Bowers BJ, Charmaz K, et al., eds. Developing Grounded Theory: The Second Generation Revisited. 2nd ed. Routledge; 2021.

178. Pitkala KH, Juola AL, Soini H, et al. Reducing inappropriate, anticholinergic and psychotropic drugs among older residents in assisted living facilities: study protocol for a randomized controlled trial. Trials. 2012;13(1):85. doi:10.1186/1745-6215-1385

179. Gerlach LB, Kales HC. Pharmacological Management of Neuropsychiatric Symptoms of Dementia. Curr Treat Options Psychiatry. 2020;7(4):489-507. doi:10.1007/s40501-020-00233-9

180. Ijaopo EO. Dementia-related agitation: a review of non-pharmacological interventions and analysis of risks and benefits of pharmacotherapy. Transl Psychiatry. 2017;7(10):e1250. doi:10.1038/tp.2017.199 
181. Parsons C. Polypharmacy and inappropriate medication use in patients with dementia: an underresearched problem. Ther Adv Drug Saf. 2017;8(1):31-46. doi: $10.1177 / 2042098616670798$

182. Mangin D, Bahat G, Golomb BA, et al. International Group for Reducing Inappropriate Medication Use \& Polypharmacy (IGRIMUP): Position Statement and 10 Recommendations for Action. Drugs Aging. 2018;35(7):575-587. doi:10.1007/s40266-018-0554-2

183. Ramsey CM, Gnjidic D, Agogo GO, Allore H, Moga D. Longitudinal patterns of potentially inappropriate medication use following incident dementia diagnosis. Alzheimers Dement Transl Res Clin Interv. 2018;4(1):1-10. doi:10.1016/j.trci.2017.10.008

184. Werner RM, Konetzka RT, Kim M. Quality Improvement Under Nursing Home Compare: The Association Between Changes in Process and Outcome Measures. Med Care. 2013;51(7):582-588. doi:10.1097/MLR.0b013e31828dbae4

185. Tamara Konetzka R, Yan K, Werner RM. Two Decades of Nursing Home Compare: What Have We Learned? Med Care Res Rev. Published online June 13, 2020:1077558720931652. doi:10.1177/1077558720931652

186. Castle NG, Ferguson JC. What Is Nursing Home Quality and How Is It Measured? The Gerontologist. 2010;50(4):426-442. doi:10.1093/geront/gnq052

187. Temkin-Greener H, Zheng NT, Cai S, Zhao H, Mukamel DB. Nursing Home Environment and Organizational Performance: Association With Deficiency Citations. Med Care. 2010;48(4):357-364. doi:10.1097/MLR.0b013e3181ca3d70

188. June JW, Meng H, Dobbs D, Hyer K. Using Deficiency Data to Measure Quality in Assisted Living Communities: A Florida Statewide Study. J Aging Soc Policy. 2020;32(2):125-140. doi:10.1080/08959420.2018.1563471

189. Castle NG, Wagner LM, Ferguson JC, Handler SM. Nursing Home Deficiency Citations for Safety. J Aging Soc Policy. 2010;23(1):34-57. doi:10.1080/08959420.2011.532011

190. Lerner NB, Johantgen M, Trinkoff AM, Storr CL, Han K. Are Nursing Home Survey Deficiencies Higher in Facilities With Greater Staff Turnover. J Am Med Dir Assoc. 2014;15(2):102-107. doi:10.1016/j.jamda.2013.09.003

191. Lepore MJ, Lima JC, Miller SC. Nursing Home Culture Change Practices and Survey Deficiencies: A National Longitudinal Panel Study. The Gerontologist. 2020;60(8):1411-1423. doi:10.1093/geront/gnaa063

192. Castle NG, Engberg JB. Nursing Home Deficiency Citations for Medication Use. J Appl Gerontol. 2007;26(2):208-232. doi:10.1177/0733464807300223

193. Vogelsmeier A. Medication Administration in Nursing Homes: RN Delegation to Unlicensed Assistive Personnel. J Nurs Regul. 2011;2(3):49-55. doi:10.1016/S21558256(15)30273-8

194. Young HM, Gray SL, McCormick WC, et al. Types, Prevalence, and Potential Clinical Significance of Medication Administration Errors in Assisted Living. J Am Geriatr Soc. 2008;56(7):1199-1205. doi:https://doi.org/10.1111/j.15325415.2008.01754.x 
195. Trinkoff AM, Lerner NM, Storr CL, Yoon JM, Yang BK, Han K. Nursing Staff Availability and Other Facility Characteristics in Relation to Assisted Living Care Deficiencies. J Nurs Regul. 2019;10(1):21-27. doi:10.1016/S2155-8256(19)30079-1

196. Zimmerman S, Love K, Sloane PD, Cohen LW, Reed D, Carder PC. Medication Administration Errors in Assisted Living: Scope, Characteristics, and the Importance of Staff Training. J Am Geriatr Soc. 2011;59(6):1060-1068. doi:https://doi.org/10.1111/j.1532-5415.2011.03430.x

197. Smith L, Carder P, Bucy T, et al. Connecting policy to licensed assisted living communities, introducing health services regulatory analysis. Health Serv Res. in press;n/a(n/a). doi:https://doi.org/10.1111/1475-6773.13616

198. Safety, Oversight, and Quality Unit. Compliance Framework Guide (Residential Care and Assisted Living. Oregon Department of Human Services, Office of Aging and People with Disabilities; 2019. https://www.oregon.gov/dhs/PROVIDERSPARTNERS/LICENSING/CBC/GuidesPubs/CBC\%20Regulatory\%20Compliance\% 20Framework\%20Guide.pdf

199. Vaismoradi M, Fredriksen Moe C, Vizcaya-Moreno MF, Paal P. Administration of pro re nata medications by the nurse to incapacitated patients: An ethical perspective. Clin Ethics. Published online July 23, 2021:14777509211034146. doi:10.1177/14777509211034146

200. Mitty E. Medication Management in Assisted Living: A National Survey of Policies and Practices. J Am Med Dir Assoc. 2009;10(2):107-114. doi:10.1016/j.jamda.2008.08.006

201. Kelly C, Craft Morgan J, Kemp CL, Deichert J. A Profile of the Assisted Living Direct Care Workforce in the United States. J Appl Gerontol. 2020;39(1):16-27. doi: $10.1177 / 0733464818757000$

202. Hrybyk RL, Frankowski AC, Nemec M, Peeples AD. "It's a lot!" the universal worker model and dementia care in assisted living. Geriatr Nur (Lond). 2021;42(1):233-239. doi:10.1016/j.gerinurse.2020.08.006

203. Carder PC, Zimmerman S, Schumacher JG. Understanding the Intersection of Individual Needs and Choices With Organizational Practices: The Case of Medication Management in Assisted Living. The Gerontologist. 2009;49(4):463473. doi:10.1093/geront/gnp063

204. Oregon Department of Human Services, Aging and People with Disabilities. Licensed Long-term Care Settings Search. Oregon.gov. Published 2019. Accessed October 16, 2021. https://ttclicensing.oregon.gov/

205. ATLAS.ti Scientific Software Development GmbH. ATLAS.Ti.; 2018.

206. Oregon Office of Rural Health. ORH Service Areas. Published 2020. www.ohsu.edu/oregon-office-of-rural-health/orh-service-areas

207. Oregon Secretary of State. Find a Business. Published 2020. https://sos.oregon.gov/business/Pages/find.aspx

208. Guest G, MacQueen K, Namey E. Applied Thematic Analysis. SAGE Publications, Inc.; 2012. doi:10.4135/9781483384436

209. King N, Brooks J. Thematic Analysis in Organisational Research. In: The SAGE Handbook of Qualitative Business and Management Research Methods: Methods 
and Challenges. SAGE Publications Ltd; 2018:219-236.

doi:10.4135/9781526430236.n14

210. Fereday J, Muir-Cochrane E. Demonstrating Rigor Using Thematic Analysis: A Hybrid Approach of Inductive and Deductive Coding and Theme Development. Int J Qual Methods. 2006;5(1):80-92. doi:10.1177/160940690600500107

211. Neuendorf KA. Content analysis and thematic analysis. In: Brough P, ed. Advanced Research Methods for APplied Psychology: Design, Analysis, and Reporting. Routledge Taloyr and Francis Groups; 2019:211-223.

212. Calsolaro V, Antognoli R, Okoye C, Monzani F. The Use of Antipsychotic Drugs for Treating Behavioral Symptoms in Alzheimer's Disease. Front Pharmacol. 2019;10(1465):1-8. doi:10.3389/fphar.2019.01465

213. Gray SL, Odegard PS, Sales AE, Young HM, Sullivan JH, Hedrick SC. Quality of Medication Records and Use of Pharmacy Resources in Community Residential Care Facilities. Ann Pharmacother. 2006;40(5):894-899. doi:10.1345/aph.1G585

214. Procter R, Wherton J, Greenhalgh T. Hidden Work and the Challenges of Scalability and Sustainability in Ambulatory Assisted Living. ACM Trans Comput-Hum Interact. 2018;25(2):11:1-11:26. doi:10.1145/3185591

215. Spetz J, Stone RI, Chapman SA, Bryant N. Home And Community-Based Workforce For Patients With Serious Illness Requires Support To Meet Growing Needs. Health Aff (Millwood). 2019;38(6):902-909. doi:10.1377/hlthaff.2019.00021

216. Stone R, Harahan MF. Improving The Long-Term Care Workforce Serving Older Adults. Health Aff (Millwood). 2010;29(1):109-115. doi:10.1377/hlthaff.2009.0554

217. Spellbring AM, Ryan JW. Medication Administration by Unlicensed Caregivers: A Model Program. J Gerontol Nurs. 2003;29(6):48-54. doi:10.3928/0098-913420030601-10

218. Reinhard SC, Young HM, Kane RA, Quinn WV. Nurse delegation of medication administration for older adults in assisted living. Nurs Outlook. 2006;54(2):74-80. doi:10.1016/j.outlook.2005.05.008

219. Beeber AS, Zimmerman S, Mitchell CM, Reed D. Staffing and Service Availability in Assisted Living: The Importance of Nurse Delegation Policies. J Am Geriatr Soc. 2018;66(11):2158-2166. doi:https://doi.org/10.1111/jgs.15580

220. Witry MJ, Doucette WR. Community pharmacists, medication monitoring, and the routine nature of refills: A qualitative study. J Am Pharm Assoc. 2014;54(6):594603. doi:10.1331/JAPhA.2014.14065

221. Cross AJ, Le VJ, George J, Woodward MC, Elliott RA. Stakeholder perspectives on pharmacist involvement in a memory clinic to review patients' medication management and assist with deprescribing. Res Soc Adm Pharm. 2020;16(5):681688. doi:10.1016/j.sapharm.2019.08.024

222. Alabama Department of Public Health. Assisted Living Facilities.; 2019. https://www.alabamapublichealth.gov/providerstandards/assets/alfruleseff04062019. pdf

223. Idaho Department of Health and Welfare. Residential Assisted Living Facilities. https://adminrules.idaho.gov/rules/current/16/160322.pdf 
224. Bowblis JR, Crystal S, Intrator O, Lucas JA. Response to Regulatory Stringency: The Case of Antipsychotic Medication Use in Nursing Homes. Health Econ. 2012;21(8):977-993. doi:https://doi.org/10.1002/hec.1775

225. Bowblis JR, Lucas JA, Brunt C. The Effects of Antipsychotic Quality Reporting on Antipsychotic and Psychoactive Medication Use. Health Serv Res. 2015;50(4):10691087. doi:10.1111/1475-6773.12281

226. Davila H, Shippee TP, Park YS, Brauner D, Werner RM, Konetzka RT. Inside the Black Box of Improving on Nursing Home Quality Measures. Med Care Res Rev. Published online September 28, 2020:1077558720960326. doi:10.1177/1077558720960326

227. Konetzka RT, Brauner DJ, Shega J, Werner RM. The Effects of Public Reporting on Physical Restraints and Antipsychotic Use in Nursing Home Residents with Severe Cognitive Impairment. J Am Geriatr Soc. 2014;62(3):454-461. doi:10.1111/jgs.12711

228. Sengupta M, Caffrey C. Characteristics of Residential Care Communities by Percentage of Resident Population Diagnosed with Dementia: United States, 2016. U.S. Department of Health and Human Services, Centers for Disease Control and Prevention, National Center for Health Statistics; 2020. https://www.cdc.gov/nchs/data/nhsr/NHSR148-508.pdf

229. Delfino LL, Komatsu RS, Komatsu C, et al. Neuropsychiatric symptoms associated with family caregiver burden and depression. Dement Amp Neuropsychol. 2021;15(1):128-135. doi:10.1590/1980-57642021dn15-010014

230. Zimmerman S, Sloane PD, Williams CS, et al. Dementia Care and Quality of Life in Assisted Living and Nursing Homes. The Gerontologist. 2005;45(suppl_1):133-146. doi:10.1093/geront/45.suppl_1.133

231. Steinberg M, Lyketsos CG. Atypical Antipsychotic Use in Patients With Dementia: Managing Safety Concerns. Am J Psychiatry. 2012;169(9):900-906. doi:10.1176/appi.ajp.2012.12030342

232. Muench J, Hamer AM. Adverse Effects of Antipsychotic Medications. Am Fam Physician. 2010;81(5):617-622.

233. Tampi RR, Tampi DJ, Balachandran S, Srinivasan S. Antipsychotic use in dementia: a systematic review of benefits and risks from meta-analyses. Ther Adv Chronic Dis. 2016;7(5):229-245. doi:10.1177/2040622316658463

234. Bishara D, Perera G, Harwood D, et al. Centrally-acting anticholinergic drugsassociations with mortality, hospitalisation and cognitive decline following dementia diagnosis in people receiving antidepressant and antipsychotic drugs. Aging Ment Health. 2021;0(0):1-9. doi:10.1080/13607863.2021.1947967

235. Vigen CLP, Mack WJ, Keefe RSE, et al. Cognitive Effects of Atypical Antipsychotic Medications in Patients With Alzheimer's Disease: Outcomes From CATIE-AD. Am J Psychiatry. 2011;168(8):831-839. doi:10.1176/appi.ajp.2011.08121844

236. Shippee TP, Henning-Smith C, Kane RL, Lewis T. Resident- and Facility-Level Predictors of Quality of Life in Long-Term Care. The Gerontologist. 2015;55(4):643-655. doi:10.1093/geront/gnt148 
237. Zubritsky C, Abbott KM, Hirschman KB, Bowles KH, Foust JB, Naylor MD. Health-related Quality of Life: Expanding a Conceptual Framework to Include Older Adults Who Receive Long-term Services and Supports. The Gerontologist. 2013;53(2):205-210. doi:10.1093/geront/gns093

238. Zimmerman S, Cohen LW, Washington T, Ward K, Girogio P. Measures and Instruments for Quality Improvement in Assisted Living. University of North Carolina at Chapel Hill; 2016. https://www.theceal.org/images/Measures-andInstruments-for-Quality-Improvement-in-Assisted-Living_Final-Report.pdf

239. Shippee TP, Akosionu O, Brasure M, Beebe T. Literature Review and Environmental Scan: Identifying Quality Measures in Assisted Living: A Report to the Minnesota Department of Human Services. University of Minnesota School of Public Health; 2019. https://mn.gov/dhs/assets/UMN-assisted-living-qualityreport_tcm1053-393870.pdf

240. Donabedian A. The Quality of Care: How Can It Be Assessed? JAMA. 1988;260(12):1743-1748. doi:10.1001/jama.1988.03410120089033

241. Donabedian A. The role of outcomes in quality assessment and assurance. $Q R B$ Qual Rev Bull. 1992;18(11):356-360. doi:10.1016/s0097-5990(16)30560-7

242. Donabedian A. Evaluating the Quality of Medical Care -. Milbank Q. 2005;83(4):691-729. doi:10.1111/j.1468-0009.2005.00397.x

243. Berwick D, Fox DM. "Evaluating the Quality of Medical Care": Donabedian's Classic Article 50 Years Later -. Milbank Q. 2016;94(2):237-241. doi:10.1111/14680009.12189

244. Megivern DM, McMillen JC, Proctor EK, Striley CLW, Cabassa LJ, Munson MR. Quality of care: Expanding the social work dialogue. Soc Work. 2007;52(2):115-124. doi:10.1093/sw/52.2.115

245. Mollica RL. Aging in Place in Assisted Living: State Regulations and Practice. American Seniors Housing Association; 2005. http://www.canhr.org/reports/2005/ASHA_paper-AIP_final.pdf

246. Carder PC, Tunalilar O, Elliott S, Dys S, Neal MB. Community-Based Care Resident and Community Characteristics Report: Assisted Living, Residential Care, Memory Care, Spring 2017.; 2017. https://www.pdx.edu/institute-onaging/sites/g/files/znldhr3046/files/2020-07/2017_CBC_AnnualReport_AL-RCMC.pdf

247. Carder PC, Tunalilar O, Elliott S, Dys S. 2018 Community-Based Care: Resident and Community Characteristics Report on Assisted Living, Residential Care, Memory Care. Institute on Aging, Portland State University; 2018. https://www.pdx.edu/institute-on-aging/sites/g/files/znldhr3046/files/202007/2018_CBC_AnnualReport_AL-RC-MC.pdf

248. Han K, Trinkoff AM, Storr CL, Lerner N, Yang BK. Variation Across U.S. Assisted Living Facilities: Admissions, Resident Care Needs, and Staffing. J Nurs Scholarsh. 2017;49(1):24-32. doi:https://doi.org/10.1111/jnu.12262

249. Wolff JL, Spillman B. Older Adults Receiving Assistance With Physician Visits and Prescribed Medications and Their Family Caregivers: Prevalence, Characteristics, 
and Hours of Care. J Gerontol Ser B. 2014;69(Suppl_1):S65-S72.

doi:10.093/geronb/gbu119

250. Frankenthal D, Zandman-Goddard G, Ben-Muvhar Y, Porat-Katz BS. The impact of facility characteristics on the use of antipsychotic medications in nursing homes: a cross-sectional study. Isr J Health Policy Res. 2016;5(1):12. doi:10.1186/s13584016-0070-y

251. Executive Office of the President, Office of Budget and Management, Office of Information and Regulatory Affairs. Revisions to the Standards for the Classification of Federal Data on Race and Ethnicity. Vol 58782.; 1997. Accessed March 17, 2021. https://www.govinfo.gov/content/pkg/FR-1997-10-30/pdf/97-28653.pdf

252. Rabe-Hesketh S, Skrondal A. Multilevel and Longitudinal Modeling Using Stata, Second Edition. Stata Press; 2008.

253. StataCorp. Stata Statistical Software: Release 15. StataCorp LLC; 2017.

254. Carder PC, Elliott S, Tunalilar O, Dys S. 2018 Adult Foster Home Resident and Community Characteristics Report on Adult Foster Homes. Institute on Aging, Portland State University; 2018. https://www.pdx.edu/institute-onaging/sites/g/files/znldhr3046/files/2020-07/2018_CBC_AnnualReport_AFH.pdf

255. Mueller C, John C, Perera G, Aarsland D, Ballard C, Stewart R. Antipsychotic use in dementia: the relationship between neuropsychiatric symptom profiles and adverse outcomes. Eur J Epidemiol. 2021;36(1):89-101. doi:10.1007/s10654-02000643-2

256. Fashaw S, Chisholm L, Mor V, et al. Inappropriate Antipsychotic Use: The Impact of Nursing Home Socioeconomic and Racial Composition. J Am Geriatr Soc. 2020;68(3):630-636. doi:https://doi.org/10.1111/jgs.16316

257. Mor V, Zinn J, Angelelli J, Teno JM, Miller SC. Driven to Tiers: Socioeconomic and Racial Disparities in the Quality of Nursing Home Care. Milbank $Q$. 2004;82(2):227-256. doi:https://doi.org/10.1111/j.0887-378X.2004.00309.x

258. Thach N, Wiener J. An Overview of Long-Term Services and Supports and Medicaid: Final Report. RTI International; 2018. https://aspe.hhs.gov/basicreport/overview-long-term-services-and-supports-and-medicaid-final-report

259. Bowblis JR, Applebaum R. How Does Medicaid Reimbursement Impact Nursing Home Quality? The Effects of Small Anticipatory Changes. Health Serv Res. 2017;52(5):1729-1748. doi:https://doi.org/10.1111/1475-6773.12553

260. Intrator O, Mor V. Effect of State Medicaid Reimbursement Rates on Hospitalizations from Nursing Homes. J Am Geriatr Soc. 2004;52(3):393-398. doi:https://doi.org/10.1111/j.1532-5415.2004.52111.x

261. Intrator O, Schleinitz M, Grabowski DC, Zinn J, Mor V. Maintaining Continuity of Care for Nursing Home Residents: Effect of States' Medicaid Bed-Hold Policies and Reimbursement Rates. Health Serv Res. 2009;44(1):33-55. doi:https://doi.org/10.1111/j.1475-6773.2008.00898.x

262. Oregon Department of Human Services, Office of Aging and People with Disabilities. Rate Schedule. Oregon Department of Human Services, Office of Aging and People with Disabilities; 2018. https://www.oregon.gov/DHS/PROVIDERS- 
PARTNERS/LICENSING/AdminAlerts/Medicaid\%20Rate\%20Changes\%20Effecti ve\%20July\%201\%202018\%20SUPPLEMENTAL.pdf

263. Comparison of Long-Term Care Settings in Oregon, 2019. Oregon Department of Human Services, Office of Aging and People with Disabilities; 2019. https://www.oregon.gov/dhs/SENIORS-DISABILITIES/Documents/2019-FacilityComparison-Table.pdf

264. Lepore M, Knowles M, Porter KA, O’Keeffe J, Wiener J. Medicaid Beneficiaries' Access to Residential Care Settings. J Hous Elder. 2017;31(4):351-366. doi:10.1080/02763893.2017.1335669

265. Weech-Maldonado R, Lord J, Pradhan R, et al. High Medicaid Nursing Homes: Organizational and Market Factors Associated With Financial Performance. Inq J Med Care Organ Provis Financ. 2019;56:0046958018825061. doi:10.1177/0046958018825061

266. Sharma H, Perraillon MC, Werner RM, Grabowski DC, Konetzka RT. Medicaid and Nursing Home Choice: Why Do Duals End Up in Low-Quality Facilities? J Appl Gerontol. 2020;39(9):981-990. doi:10.1177/0733464819838447

267. Hernandez M. Assisted living and residential care in Oregon: two decades of state policy, supply, and Medicaid participation trends. The Gerontologist. 2007;47 Spec No 3:118-124. doi:10.1093/geront/47.supplement_1.118

268. Fabius CD, Cornell PY, Zhang W, Thomas KS. State Medicaid Financing and Access to Large Assisted Living Settings for Medicare-Medicaid Dual-Eligibles. Med Care Res Rev. Published online January 19, 2021:1077558720987666. doi:10.1177/1077558720987666

269. Eckenweiler LA. Realizing Justice Globally in Long-Term Care. In: Long-Term Care, Globalization, and Justice. Johns Hopkins University Press; 2012:87-106.

270. Jackson BE, Pederson A, Armstrong P, et al. "Quality Care is Like a Carton of Eggs": Using a gender-based diversity analysis to assess quality of health care. Can Woman Stud. 2004;24(1):15-22.

271. Hua CL, Cornell PY, Zimmerman S, Winfree J, Thomas KS. Trends in Serious Mental Illness in US Assisted Living Compared to Nursing Homes and the Community: 2007-2017. Am J Geriatr Psychiatry Off J Am Assoc Geriatr Psychiatry. 2021;29(5):434-444. doi:10.1016/j.jagp.2020.09.011

272. Fashaw-Walters SA, McCreedy E, Bynum JPW, Thomas KS, Shireman TI. Disproportionate increases in schizophrenia diagnoses among Black nursing home residents with ADRD. J Am Geriatr Soc. Published online September 30, 2021. doi:10.1111/jgs.17464

273. Eckenweiler LA. An Ecological Ethic. In: Long-Term Care, Globalization, and Justice. Johns Hopkins University Press; 2012:70-86.

274. Hanefeld J, Powell-Jackson T, Balabanova D. Understanding and measuring quality of care: dealing with complexity. Bull World Health Organ. 2017;95(5):368-374. doi:10.2471/BLT.16.179309

275. Kendall T. The rise and fall of the atypical antipsychotics. Br J Psychiatry. 2011;199(4):266-268. doi:10.1192/bjp.bp.110.083766 
276. Rodwin MA. Rooting Out Institutional Corruption to Manage Inappropriate OffLabel Drug Use. J Law Med Ethics. 2013;41(3):654-664. doi:10.1111/jlme.12075

277. Tun SM, Murman DL, Long HL, Colenda CC, von Eye A. Predictive Validity of Neuropsychiatric Subgroups on Nursing Home Placement and Survival in Patients With Alzheimer Disease. Am J Geriatr Psychiatry. 2007;15(4):314-327. doi:10.1097/01.JGP.0000239263.52621.97

278. Sagud M, Tudor L, Pivac N. Personalized treatment interventions: nonpharmacological and natural treatment strategies in Alzheimer's disease. Expert Rev Neurother. 2021;0(0):1-19. doi:10.1080/14737175.2021.1906223

279. WHO Collaborating Centre for Drug Statistics Methodology. ATC/DDD IndexNervous System. WHO Collaborating Centre for Drug Statistics Methodology. Published December 17, 2020. Accessed October 17, 2021. https://www.whocc.no/atc_ddd_index/

280. Welberry HJ, Jorm LR, Schaffer AL, et al. Psychotropic medicine prescribing and polypharmacy for people with dementia entering residential aged care: the influence of changing general practitioners. Med J Aust. n/a(n/a). doi:10.5694/mja2.51153

281. Bangash A, Stubbs R, Khan F, Samnani S, Aziz H, Mitra M. Association between antipsychotics and adverse outcomes in dementia. Prog Neurol Psychiatry. 2017;21(4):20-26. doi:https://doi.org/10.1002/pnp.482

282. Desai AK, McFadden SH. Reducing Antipsychotic Use in Long Term Care Residents: Addressing Psychological and Spiritual Needs of Residents With Dementia Is Crucial. J Am Med Dir Assoc. 2013;14(3):224-225. doi:10.1016/j.jamda.2012.12.008

283. Bonner AF, Field TS, Lemay CA, et al. Rationales that Providers and Family Members Cited for the Use of Antipsychotic Medications in Nursing Home Residents with Dementia. J Am Geriatr Soc. 2015;63(2):302-308. doi:https://doi.org/10.1111/jgs.13230

284. Green AR, Lee P, Reeve E, et al. Clinicians' Perspectives on Barriers and Enablers of Optimal Prescribing in Patients with Dementia and Coexisting Conditions. J Am Board Fam Med. 2019;32(3):383-391. doi:10.3122/jabfm.2019.03.180335

285. Walsh KA, Dennehy R, Sinnott C, et al. Influences on Decision-Making Regarding Antipsychotic Prescribing in Nursing Home Residents With Dementia: A Systematic Review and Synthesis of Qualitative Evidence. J Am Med Dir Assoc. 2017;18(10):897.e1-897.e12. doi:10.1016/j.jamda.2017.06.032

286. Aerts L, Cations M, Harrison F, et al. Why deprescribing antipsychotics in older people with dementia in long-term care is not always successful: Insights from the HALT study. Int J Geriatr Psychiatry. 2019;34(11):1572-1581. doi:https://doi.org/10.1002/gps.5167

287. Harper AE, Terhorst L, Moscirella M, Turner RL, Piersol CV, Leland NE. The experiences, priorities, and perceptions of informal caregivers of people with dementia in nursing homes: A scoping review. Dementia. Published online April 24, 2021:14713012211012606. doi:10.1177/14713012211012606 
288. de Oliveira AM, Radanovic M, de Mello PCH, et al. Nonpharmacological Interventions to Reduce Behavioral and Psychological Symptoms of Dementia: A Systematic Review. BioMed Res Int. 2015;2015:218980. doi:10.1155/2015/218980

289. Carder PC, Schumacher JG, Zimmerman S, Sloane PD. Medication Management: Integrating the Social and Medical Models. :5.

290. Oakes CE, Sheehan NW. Autonomy in Assisted Living: Employees' Perspectives. $J$ Hous Elder. 2012;26(4):317-337. doi:10.1080/02763893.2011.649828

291. Rantsi M, Hyttinen V, Jyrkkä J, Vartiainen AK, Kankaanpää E. Process evaluation of implementation strategies to reduce potentially inappropriate medication prescribing in older population: a scoping review. Res Soc Adm Pharm. Published online April 18, 2021. doi:10.1016/j.sapharm.2021.04.012

292. Theurer K, Mortenson WB, Stone R, Suto M, Timonen V, Rozanova J. The need for a social revolution in residential care. J Aging Stud. 2015;35:201-210. doi:10.1016/j.jaging.2015.08.011

293. Boumans J, van Boekel LC, Baan CA, Luijkx KG. How Can Autonomy Be Maintained and Informal Care Improved for People With Dementia Living in Residential Care Facilities: A Systematic Literature Review. The Gerontologist. 2019;59(6):e709-e730. doi:10.1093/geront/gny096

294. Brodaty H, Aerts L, Harrison F, et al. Antipsychotic Deprescription for Older Adults in Long-term Care: The HALT Study. J Am Med Dir Assoc. 2018;19(7):592-600.e7. doi:10.1016/j.jamda.2018.05.002

295. Beeber AS, Cohen LW, Zimmerman S, et al. Differences in Assisted Living Staff Perceptions, Experiences, and Attitudes. J Gerontol Nurs. 2014;40(1):41-49. doi:10.3928/00989134-20130731-03

296. Ervin K, Cross M, Koschel A. Barriers to managing behavioural and psychological symptoms of dementia: Staff perceptions. Collegian. 2014;21(3):201-207. doi:10.1016/j.colegn.2013.04.002

297. Foley KL, Sudha S, Sloane PD, Gold DT. Staff Perceptions of Successful management of severe behavioral problems in dementia special care units. Dementia. 2003;2(1):105-124. doi:10.1177/1471301203002001998

298. Hughes JC, Baldwin C. Ethical Issues in Dementia Care: Making Difficult Decisions. Jessica Kingsley Publishers; 2006.

299. Jennings B. Agency and Moral Relationship in Dementia. Metaphilosophy. 2009;40(3-4):425-437. doi:10.1111/j.1467-9973.2009.01591.x

300. Agrawal AK, Gowda M, Achary U, Gowda GS, Harbishettar V. Approach to Management of Wandering in Dementia: Ethical and Legal Issue. Indian J Psychol Med. Published online September 21, 2021:02537176211030979. doi: $10.1177 / 02537176211030979$

301. Carpenter WT, Buchanan RW. Antipsychotic Medications: Flawed Concepts and Ethics. Schizophr Bull. 2020;46(5):1030-1031. doi:10.1093/schbul/sbaa076

302. Treloar A, Crugel M, Prasanna A, et al. Ethical dilemmas: should antipsychotics ever be prescribed for people with dementia? Br J Psychiatry. 2010;197(2):88-90. doi:10.1192/bjp.bp.109.076307 
303. Di Pietro N. Chapter 1 - A Brief History of the Science and Ethics of Antipsychotics and Off-Label Prescribing. In: Di Pietro N, Illes J, eds. The Science and Ethics of Antipsychotic Use in Children. Academic Press; 2015:1-12. doi:10.1016/B978-0-12800016-8.00001-5

304. Harding R, Peel E. 'He was like a zombie': Off-label prescription of antipsychotic drugs in dementia. Med Law Rev. 2013;21(2):243-277. doi:10.1093/medlaw/fws029

305. Ihara $\mathrm{H}$, Arai H. Ethical dilemma associated with the off-label use of antipsychotic drugs for the treatment of behavioral and psychological symptoms of dementia. Psychogeriatrics. 2008;8(1):32-37. doi:10.1111/j.1479-8301.2007.00215.x

306. Crystal S, Olfson M, Huang C, Pincus H, Gerhard T. Broadened Use Of Atypical Antipsychotics: Safety, Effectiveness, And Policy Challenges. Health Aff (Millwood). Published online July 21, 2009. doi:10.1377/hlthaff.28.5.w770

307. Pijl-Zieber EM, Awosoga O, Spenceley S, Hagen B, Hall B, Lapins J. Caring in the wake of the rising tide: Moral distress in residential nursing care of people living with dementia. Dementia. 2018;17(3):315-336. doi:10.1177/1471301216645214

308. Colón-Emeric CS, Plowman D, Bailey D, et al. Regulation and Mindful Resident Care in Nursing Homes. Qual Health Res. 2010;20(9):1283-1294. doi:10.1177/1049732310369337

309. Elvish R, James I, Milne D. Lying in dementia care: an example of a culture that deceives in people's best interests. Aging Ment Health. 2010;14(3):255-262. doi:10.1080/13607861003587610

310. Casey D, Lynch U, Murphy K, et al. Telling a 'good or white lie': The views of people living with dementia and their carers. Dementia. 2020;19(8):2582-2600. doi:10.1177/1471301219831525

311. Talerico KA, O’Brien JA, Swafford KL. Person-centered care. An important approach for 21 st century health care. J Psychosoc Nurs Ment Health Serv. 2003;41(11):12-16.

312. Tarzia L, Fetherstonhaugh D, Bauer M, Beattie E, Nay R. "We Have to Work Within the System!": Staff Perceptions of Organizational Barriers to Decision Making for Older Adults With Dementia in Australian Aged Care Facilities. Res Gerontol Nurs. 2015;8(6):286-292. doi:10.3928/19404921-20150413-01

313. Rosenthal M, Poling J, Wec A, Connolly E, Angell B, Crystal S. "Medication Is Just One Piece of the Whole Puzzle": How Nursing Homes Change Their Use of Antipsychotic Medications. J Appl Gerontol. Published online September 30, 2020:0733464820958919. doi:10.1177/0733464820958919

314. Simmons SF, Bonnett KR, Hollingsworth E, et al. Reducing Antipsychotic Medication Use in Nursing Homes: A Qualitative Study of Nursing Staff Perceptions. The Gerontologist. 2018;58(4):e239-e250. doi:10.1093/geront/gnx083

315. Sikorska-Simmons E. Linking Resident Satisfaction to Staff Perceptions of the Work Environment in Assisted Living: A Multilevel Analysis. The Gerontologist. 2006;46(5):590-598. doi:10.1093/geront/46.5.590

316. Sikorska-Simmons E, Wright JD. Determinants of Resident Autonomy in Assisted Living Facilities: A Review of the Literature. Care Manag J. 2007;8(4):187-193. doi:10.1891/152109807782590646 
317. Comas-Herrera A, Zalakain J, Lemmon E, et al. Mortality Associated with COVID19 Outbreaks in Care Homes: Early International Evidence.; 2021. Accessed October 11, 2021. https://ltccovid.org/2020/04/12/mortality-associated-with-covid19-outbreaks-in-care-homes-early-international-evidence/

318. Dys S, Winfree J, Carder P, Zimmerman S, Thomas KS. Coronavirus Disease 2019 Regulatory Response in United States-Assisted Living Communities: Lessons Learned. Front Public Health. 2021;9:491. doi:10.3389/fpubh.2021.661042

319. Kyler-Yano JZ, Tunalilar O, Hasworth S, et al. "What Keeps Me Awake at Night": Assisted Living Administrator Responses to COVID-19. The Gerontologist. 2021;(gnab106). doi:10.1093/geront/gnab106

320. Rose G. Sick individuals and sick populations. Int J Epidemiol. 2001;30(3):427-432. doi:10.1093/ije/30.3.427

321. Krieger N. Who and what is a "population"? Historical debates, current controversies, and implications for understanding "population health" and rectifying health inequities. Milbank Q. 2012;90(4):634-681. doi:10.1111/j.14680009.2012.00678.x

322. Diez-Roux AV. Bringing context back into epidemiology: variables and fallacies in multilevel analysis. Am J Public Health. 1998;88(2):216-222. doi:10.2105/ajph.88.2.216

323. Roux AVD. A glossary for multilevel analysis. J Epidemiol Community Health. 2002;56(8):588-594. doi:10.1136/jech.56.8.588

324. Assisted Living Workgroup. Assuring Quality in Assisted Living: Guidelines for Federal and State Policy, State Regulation, and Operations.; 2003.

325. Cirka C, Messikomer C. Behind the Facade: Aligning Artifacts, Values, and Assumptions in Assisted Living. Bus Prof Ethics J. 2012;31(1):79-107. doi:10.5840/bpej20123114

326. Mathar T. Review Essay: Making a Mess with Situational Analysis? Forum Qual Sozialforschung Forum Qual Soc Res. 2008;9(2). doi:10.17169/fqs-9.2.432

327. Young SL, Taylor M, Lawrie SM. "First do no harm." A systematic review of the prevalence and management of antipsychotic adverse effects. $J$ Psychopharmacol (Oxf). 2015;29(4):353-362. doi:10.1177/0269881114562090

328. Kemp CL, Lesandrini J, Craft Morgan J, Burgess EO. The Ethics in Long-Term Care Model: Everyday Ethics and the Unseen Moral Landscape of Assisted Living. $J$ Appl Gerontol. Published online October 14, 2021:07334648211049806. doi:10.1177/07334648211049806

329. Ball MM, Whittington FJ, Perkins MM, et al. Quality of Life in Assisted Living Facilities: Viewpoints of Residents. J Appl Gerontol. 2000;19(3):304-325. doi:10.1177/073346480001900304

330. Morgan LA, Perez R, Frankowski AC, Nemec M, Bennett CR. Mental Illness in Assisted Living: Challenges for Quality of Life and Care. J Hous Elder. 2016;30(2):185-198. doi:10.1080/02763893.2016.1162255

331. Mitchell JM, Kemp BJ. Quality of Life in Assisted Living Homes: A Multidimensional Analysis. J Gerontol Ser B. 2000;55(2):P117-P127. doi:10.1093/geronb/55.2.P117 
332. Wilkins JM. Dementia, Decision Making, and Quality of Life. AMA J Ethics. 2017;19(7):637-639. doi:10.1001/journalofethics.2017.19.7.fred1-1707

333. Bonvissutto K. Oregon lawmakers consider minimum staffing ratios for memory care communities. McKnight's Senior Living. Published April 8, 2021. Accessed October 19, 2021. https://www.mcknightsseniorliving.com/home/news/oregonlawmakers-consider-minimum-staffing-ratios-for-memory-care-communities/

334. Thomas KS, Cornell PY, Zhang W, et al. The Relationship Between States' Staffing Regulations And Hospitalizations Of Assisted Living Residents. Health Aff (Millwood). 2021;40(9):1377-1385. doi:10.1377/hlthaff.2021.00598 